UCLA/06/TEP/27

SLAC-PUB-11936

SPhT-T06/070

\title{
All One-loop Maximally Helicity Violating Gluonic Amplitudes in $\mathrm{QCD}^{*}$
}

\author{
Carola F. Berger \\ Stanford Linear Accelerator Center \\ Stanford University \\ Stanford, CA 94309, USA \\ Zvi Bern \\ Department of Physics and Astronomy, UCLA \\ Los Angeles, CA 90095-1547, USA \\ Lance J. Dixon \\ Stanford Linear Accelerator Center \\ Stanford University \\ Stanford, CA 94309, USA \\ Darren Forde and David A. Kosower \\ Service de Physique Théorique $e^{\dagger}$ CEA-Saclay \\ F-91191 Gif-sur-Yvette cedex, France
}

(Dated: July 2006)

\footnotetext{
* Research supported in part by the US Department of Energy under contracts DE-FG03-91ER40662 and DE-AC02-76SF00515

† Laboratory of the Direction des Sciences de la Matière of the Commissariat à l'Energie Atomique of France.
} 


\begin{abstract}
We use on-shell recursion relations to compute analytically the one-loop corrections to maximallyhelicity-violating $n$-gluon amplitudes in QCD. The cut-containing parts have been computed previously; our work supplies the remaining rational parts for these amplitudes, which contain two gluons of negative helicity and the rest positive, in an arbitrary color ordering. We also present formulae specific to the six-gluon cases, with helicities $(-+-+++)$ and $(-++-++)$, as well as numerical results for six, seven, and eight gluons. Our construction of the $n$-gluon amplitudes illustrates the relatively modest growth in complexity of the on-shell-recursive calculation as the number of external legs increases. These amplitudes add to the growing body of one-loop amplitudes known for all $n$, which are useful for studies of general properties of amplitudes, including their twistor-space structure.
\end{abstract}

PACS numbers: 11.15.Bt, 11.25.Db, 11.25.Tq, 11.55.Bq, 12.38.Bx 


\section{INTRODUCTION}

The forthcoming experimental program at CERN's Large Hadron Collider will place new demands on theoretical calculations. Finding and understanding new physics in this environment will require the study of processes with higher multiplicity than at the Tevatron. For example, it is important to improve our understanding of missing transverse energy in association with leptons and multiple jets, arising from Standard-Model production of $W, Z+$ multi-jets. Such event classes form backgrounds to searches for supersymmetry and other models of new electroweak physics. In order to reach the precision required by searches for and measurements of new physics, these processes need to be computed to nextto-leading order (NLO), which entails the computation of one-loop amplitudes. The crucial case of $W, Z+4$ jet production involves the computation of amplitudes with seven external particles, including the vector boson. These are challenging calculations. State-of-the-art Feynman-diagrammatic computations have only recently reached six-point amplitudes [1-5].

In this paper, we instead use on-shell methods to compute loop amplitudes. On-shell methods were first developed at loop level — in the unitarity method — providing the first practical method for obtaining complete amplitudes using previously computed on-shell amplitudes $[6-10]^{1}$. With a few exceptions $[8,13]$, applications of the unitarity method were generally restricted to supersymmetric theories or to the (poly)logarithmic part of QCD amplitudes. This limitation arose from the greater complexity of calculations using $D$ dimensional unitarity [14], required for reconstructing complete QCD amplitudes including rational terms. In the past two years a number of related techniques have emerged, including the application of maximally-helicity-violating (MHV) vertices $[15,16]$ to loop calculations $[17,18]$, and the use [19] of the holomorphic anomaly. However, these techniques also suffer from the same limitations. Recent improvements to the unitarity method [13, 20] use complex momenta within generalized unitarity [21-23], allowing, for example, a simple and purely algebraic determination of all box integral coefficients. In ref. [24], Britto, Buchbinder, Cachazo and Feng developed efficient techniques for evaluating generic one-loop unitarity

\footnotetext{
${ }^{1}$ Unitarity has of course been a fundamental concept in quantum field theory since its inception (see e.g. ref. [11]). In more recent years, it has become a practical and efficient computational method for reconstructing dimensionally regularized [12] amplitudes containing massless particles and multiple kinematic invariants.
} 
cuts, by using spinor variables and performing the cut integration via residue extraction. Applying these ideas, Britto, Feng and Mastrolia [25] computed the cut-containing terms for the most complex six-gluon helicity amplitudes, the terms with a scalar circulating in the loop and gluon helicity assignments $(-+-+-+)$ and $(--+-++)$. The cut-containing terms for other helicity configurations, and for other components of the amplitudes (within a supersymmetric decomposition [26]), were obtained in refs. [6, 7, 24, 27-29].

The first use of on-shell methods to obtain state-of-the art QCD amplitudes dates to the construction of the $Z \rightarrow 4$ parton one-loop matrix elements [21] (or equivalently, by crossing, the virtual corrections for $p p \rightarrow W, Z+2$ jets). These matrix elements have been incorporated into several numerical programs for NLO corrections to a variety of collider processes [30]. The technique used in ref. [21] was to first obtain the (poly)logarithmic terms in the amplitudes via the unitarity method. The rational terms were found by constructing functions with the proper on-shell factorization properties, by matching the known behavior as two partons become parallel (collinear singularities), or as invariants formed out of three or more partons vanish (multi-particle poles). This rational-term reconstruction method did not achieve widespread application because it was unclear how to make it systematic. In particular, as the number of external legs $n$ increases, it becomes very difficult to build properly factorizing functions; the number of candidate terms in an ansatz for the rational terms grows rapidly with $n$.

One-loop on-shell recursion relations [31, 32] provide a means for overcoming this difficulty. They allow for a practical and systematic construction of the rational terms of loop amplitudes. Such recursion relations were written down at tree level by Britto, Cachazo and Feng [33]. Their work was stimulated by the compact forms of seven- and higher-point tree amplitudes [23, 34, 35] that emerged from studying infrared consistency equations [36] for one-loop amplitudes (computed using the unitarity-based method), and by the connection between twistor space and complexified momenta noted in ref. [37]. Britto, Cachazo, Feng and Witten [38] then proved the tree-level on-shell recursion relations using a special continuation, or 'shift', of the external momenta by a complex variable $z$. The proof essentially involves only Cauchy's residue theorem, where the residues in the $z$ plane are determined by the amplitudes' factorization properties (for complex momenta). The remarkable generality and simplicity of the proof enabled widespread application at tree level [39-41], including to theories with massive particles [42-44]. 
The extension of on-shell recursive methods to one-loop amplitudes was developed in refs. [31, 32, 45-47]. The essential inputs to this approach are the cut-constructible parts of a desired amplitude — those terms containing polylogarithms, logarithms, and associated $\pi^{2}$ terms - and appropriate tree and loop amplitudes with fewer legs, which appear in factorization limits of the amplitude. Closed-form expressions were found for several sequences of one-loop amplitudes with an arbitrary number of gluons. In particular, the amplitudes for ' $M H V$ ' helicity configurations, in which two of the gluons have negative helicity and the rest have positive helicity, were computed for the special case that the two negative-helicity gluons are adjacent in the color ordering [31, 47].

In this paper, we use the same methods to compute the rational parts, and thereby the complete expressions, for all remaining $n$-gluon MHV amplitudes, those for which the two negative-helicity gluons are not color-adjacent. Under a supersymmetric decomposition [26], $n$-gluon amplitudes may be thought of as composed of $\mathcal{N}=4$ and $\mathcal{N}=1$ supersymmetric pieces together with a non-supersymmetric $(\mathcal{N}=0)$ scalar loop contribution. Using the unitarity method, the $\mathcal{N}=4 \mathrm{MHV}$ contributions were computed in ref. [6], and the $\mathcal{N}=1$ terms in ref. [7]. The polylogarithmic contributions to the scalar-loop components of the MHV amplitudes were obtained in ref. [18] using MHV vertices [15, 17]; these contributions serve as direct input to our recursive construction of the rational terms.

A number of new aspects must be considered in order to construct loop-level on-shell recursion relations. The most obvious feature is that the shifted amplitude $A(z)$ contains branch cuts in the $z$ plane. We will handle this feature as in refs. [31, 32], by subtracting the cut-containing terms in the amplitude, along with certain additional 'cut completions' that cancel spurious singularities, before applying Cauchy's theorem to the remaining rational part.

Secondly, we must ensure that the residues of all poles at finite $z$ are known. The factorization properties of amplitudes with real external momenta are well understood [6, 4850]. They completely determine the complex factorization properties of tree amplitudes [38]. However, the same statement is not true at loop level. In general, non-supersymmetric loop amplitudes contain 'unreal poles' which appear for complex momenta but are absent for real momenta. Since these unreal poles are not yet fully understood, the best strategy is to consider complex-momentum shifts that avoid channels with unknown factorization properties. As discussed in ref. [32], for $n$-gluon amplitudes the problematic channels always 
have precisely two identical-helicity gluons on one side of the factorization. In this paper, we consider MHV amplitudes with two negative-helicity gluons, and we elect to shift the momenta of these two gluons. Because these gluons are color-adjacent to positive-helicity gluons, the two-particle channels will always have opposite gluon helicities. Hence there are no problematic channels in our construction.

The straightforward application of Cauchy's theorem underlying our computation requires that the amplitude fall off as the shift parameter $z$ is taken to infinity. A more elaborate construction is needed [32] when this requirement is not satisfied. We can see from known four- and five-point one-loop amplitudes that the requirement is indeed satisfied for our choices of shift momenta. We assume that the same holds for $n>5$. We confirm this assumption at the end of the calculation, by checking factorization properties as well as reflection or 'flip' symmetries of the computed amplitudes.

In addition to determining rational terms in amplitudes efficiently, on-shell recursion relations can in some cases provide an effective way to compute the (rational) coefficients of integral functions appearing in amplitudes [29]. Indeed, this method was used to determine the coefficients of all integral functions appearing in the split-helicity $n$-gluon amplitude, for which identical helicity gluons are nearest neighbors in the color ordering.

Our interest in constructing all-multiplicity amplitudes stems partly from the desire to study the growth in complexity of the amplitudes as the number of external partons increases. A difficulty with previous computational methods has been the rather rapid growth in complexity with the number of external legs. In contrast, with our bootstrap construction we find only mild growth, at least for the MHV and split helicity configurations, allowing us to obtain closed-form analytic expressions. Furthermore, experience has shown that analytic all- $n$ expressions provide a wealth of intuition into the general structure of the scattering amplitudes.

In a very interesting series of recent papers, Xiao, Yang and Zhu have obtained the rational terms for all independent one-loop six-gluon amplitudes using Feynman diagrams, by applying spinor simplifications together with integrations that target only the rational terms $[51,52]$. When combined with the previously-obtained cut parts $[7,18,24,25,27,34]$, these results provide analytic expressions for all remaining six-gluon helicity configurations in QCD. We have compared our results for the MHV case and find complete agreement. We have also compared our results for six gluons with the numerical results of Ellis, Giele and 
Zanderighi [4] and find agreement.

This paper is organized as follows. In the next section we briefly remind the reader of our notation. We give an overview of the on-shell bootstrap approach in section III. We apply this method to the recursive construction of MHV amplitudes in section IV, giving details of the method for five and six external gluons. We also give numerical values at selected momentum configurations for the six-, seven- and eight-point amplitudes. These values can serve as a reference for future phenomenological implementations. Section V contains the all-multiplicity expression for the complete rational parts of one-loop MHV amplitudes. We summarize our results and give an outlook in section VI. In the appendices we quote previously obtained results for the (poly)logarithmic parts of MHV amplitudes. We also give analytical expressions for the newly-computed rational parts of the six-point MHV amplitudes.

\section{NOTATION}

In this paper, we shall follow the same notation as defined in section II of our companion paper [32]. We therefore refer the reader to that paper for notation. Our amplitudes will be expressed in terms of spinor inner products [53]. As is standard for one-loop QCD amplitudes, we present color-ordered amplitudes $[9,48,54,55]$. We need to present only the leading-color contributions, because the subleading-color partial amplitudes for a gluon in the loop are given by a sum over permutations of the leading-color ones [6]. (For fundamental representation particles such as quarks in the loop the leading contributions give the entire expression directly.)

We shall use the supersymmetric decomposition of $n$-gluon QCD amplitudes [7, 26] to rewrite the contributions of different spin states circulating in the loop in terms of supersymmetric and non-supersymmetric components,

$$
\begin{aligned}
A_{n ; 1}^{[1 / 2]} & =A_{n ; 1}^{\mathcal{N}=1}-A_{n ; 1}^{\mathcal{N}=0}, \\
A_{n ; 1}^{[1]} & =A_{n ; 1}^{\mathcal{N}=4}-4 A_{n ; 1}^{\mathcal{N}=1}+A_{n ; 1}^{\mathcal{N}=0} .
\end{aligned}
$$

where $A_{n ; 1}^{[J]}$ denotes an $n$-gluon amplitude with a particle of spin $J$ circulating in the loop. The non-supersymmetric amplitudes, denoted by $\mathcal{N}=0$, are just the contributions of a

complex scalar circulating in the loop, $A_{n ; 1}^{\mathcal{N}=0} \equiv A_{n ; 1}^{[0]}$. The amplitudes $A_{n ; 1}^{\mathcal{N}=1}$ and $A_{n ; 1}^{\mathcal{N}=4}$ 
represent the contribution of an $\mathcal{N}=1$ chiral multiplet consisting of a scalar and fermion, and an $\mathcal{N}=4$ multiplet consisting of one gluon, four gluinos and six real scalars, respectively.

The supersymmetric decomposition is convenient because it separates the amplitudes into pieces with differing analytic properties. The supersymmetric parts can be constructed completely from unitarity cuts in four dimensions $[6,7]$, without additional rational contributions. The polylogarithms and logarithms of the $\mathcal{N}=0$ non-supersymmetric contributions may also be computed from the four-dimensional unitarity cuts, but in this case there are in general additional non-trivial rational contributions.

The leading-color QCD amplitudes are expressible in terms of the different supersymmetric components (2.1)-(2.2) via,

$$
A_{n ; 1}^{\mathrm{QCD}}=A_{n ; 1}^{\mathcal{N}=4}-4 A_{n ; 1}^{\mathcal{N}=1}+\left(1-\epsilon \delta_{R}\right) A_{n ; 1}^{\mathcal{N}=0}+\frac{n_{f}}{N_{c}}\left(A_{n ; 1}^{\mathcal{N}=1}-A_{n ; 1}^{\mathcal{N}=0}\right)+\frac{n_{s}}{N_{c}} A_{n ; 1}^{\mathcal{N}=0}
$$

where $n_{f}$ is the number of active quark flavors in QCD. We also allow for a term proportional to the number of active fundamental representation scalars $n_{s}$, which vanishes in QCD. We regulate the infrared and ultraviolet divergences of one-loop amplitudes dimensionally. The regularization-scheme-dependent parameter $\delta_{R}$ specifies the number of helicity states of internal gluons to be $\left(2-\epsilon \delta_{R}\right)$. For the 't Hooft-Veltman scheme [12] $\delta_{R}=1$, while in the four-dimensional helicity scheme $[7,56,57] \delta_{R}=0$.

The amplitudes that we shall present in this paper are unrenormalized. To carry out $\overline{\mathrm{MS}}$ renormalization, one should subtract from the leading-color partial amplitudes $A_{n ; 1}$ the quantity,

$$
c_{\Gamma}\left[\frac{n-2}{2} \frac{1}{\epsilon}\left(\frac{11}{3}-\frac{2}{3} \frac{n_{f}}{N_{c}}-\frac{1}{3} \frac{n_{s}}{N_{c}}\right)\right] A_{n}^{\text {tree }},
$$

for $D=4-2 \epsilon$ dimensions, where,

$$
c_{\Gamma}=\frac{1}{(4 \pi)^{2-\epsilon}} \frac{\Gamma(1+\epsilon) \Gamma^{2}(1-\epsilon)}{\Gamma(1-2 \epsilon)} .
$$

\section{REVIEW OF RECURSIVE BOOTSTRAP APPROACH}

On-shell recursion relations are an efficient way to obtain the rational parts of amplitudes directly from their analytic properties. In this section, we first recapitulate the proof [38] of the tree-level recursion relations [33]. The proof relies only on elementary complex analysis and on general factorization properties satisfied by any scattering amplitude. The generality 
of the proof permits its extension to loop level, along the lines of refs. [31, 32], as we summarize in the rest of the section.

The proof of the tree-level relations employs a parameter-dependent ' $[j, l\rangle$ ' shift of two of the external massless spinors, $j$ and $l$, in an $n$-point process,

$$
\begin{aligned}
{[j, l\rangle: } & \tilde{\lambda}_{j} \\
\lambda_{l} & \rightarrow \tilde{\lambda}_{j}-z \tilde{\lambda}_{l}+z \lambda_{j}
\end{aligned}
$$

where $z$ is a complex parameter. This shift in spinor variables corresponds to shifting two of the momenta to complex values,

$$
\begin{aligned}
& k_{j}^{\mu} \rightarrow k_{j}^{\mu}(z)=k_{j}^{\mu}-\frac{z}{2}\left\langle j^{-}\left|\gamma^{\mu}\right| l^{-}\right\rangle, \\
& k_{l}^{\mu} \rightarrow k_{l}^{\mu}(z)=k_{l}^{\mu}+\frac{z}{2}\left\langle j^{-}\left|\gamma^{\mu}\right| l^{-}\right\rangle
\end{aligned}
$$

so that they remain massless, $k_{j}^{2}(z)=k_{l}^{2}(z)=0$, and overall momentum conservation is maintained. (Similar considerations apply to cases with massive particles [42-44].) An onshell amplitude containing the momenta $k_{j}$ and $k_{l}$ then becomes parameter-dependent as well,

$$
A(z)=A\left(k_{1}, \ldots, k_{j}(z), k_{j+1}, \ldots, k_{l}(z), k_{l+1}, \ldots, k_{n}\right)
$$

where the physical amplitude is recovered by taking $z=0$.

At tree level, $A(z)$ is an analytic function containing only poles, so we may exploit Cauchy's theorem to construct it from its residues. Assuming $A(z) \rightarrow 0$ as $z \rightarrow \infty$, then there is no 'surface term' in the contour integral around the circle at infinity, so it vanishes,

$$
\frac{1}{2 \pi i} \oint_{C} \frac{d z}{z} A(z)=0
$$

Evaluating the integral as a sum of residues allows us to solve for the physical amplitude $A(0)$,

$$
A(0)=-\sum_{\text {poles } \alpha} \operatorname{Res}_{z=z_{\alpha}} \frac{A(z)}{z} .
$$

At tree level, the requirement that $A(z)$ vanishes as $z \rightarrow \infty$ is rather mild; wide classes of shifts exist that satisfy this requirement [33, 38, 42].

The residues in eq. (3.5) may be obtained using the generic factorization properties that any amplitude must satisfy. Consider the factorization channel $s_{\alpha \ldots \beta} \rightarrow 0$. In this limit, the 


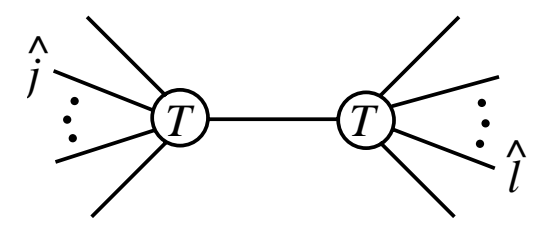

FIG. 1: Schematic representation of tree-level recursive contributions. The labels ' $T$ ' refer to tree vertices which are on-shell amplitudes. The momenta $\hat{\jmath}$ and $\hat{l}$ are shifted, on-shell momenta, evaluated according to eqs. (3.14) and (3.15).

amplitude behaves as [48],

$$
\begin{aligned}
A_{n}^{\text {tree }}\left(k_{1}, \ldots, k_{n}\right) \longrightarrow \sum_{h= \pm} A^{\text {tree }}\left(k_{\alpha}, \ldots, k_{\beta},\right. & \left.-K_{\alpha \ldots \beta}^{h}\right) \\
& \times \frac{i}{s_{\alpha \ldots \beta}} \times A^{\text {tree }}\left(k_{\beta+1}, \ldots, k_{\alpha-1}, K_{\alpha \ldots \beta}^{-h}\right),
\end{aligned}
$$

where $h= \pm 1$ is the helicity of the intermediate gluon.

Now consider the effect of the shift (3.2). If the shifted legs $j$ and $l$ are on opposite sides of the pole, as depicted in fig. 1, and leg $j$ is between legs $\alpha$ and $\beta$ in the color ordering, then under the shift,

$$
s_{\alpha \ldots \beta} \rightarrow s_{\alpha \ldots \beta}-z\left\langle j^{-}\left|K_{\alpha \ldots \beta}\right| l^{-}\right\rangle,
$$

so we have a simple pole in $z$ in this channel. Using elementary complex variable theory we may then evaluate the residue, using the rule,

$$
\operatorname{Res}_{z=z_{\alpha \beta}} \frac{f(z)}{z-z_{\alpha \beta}}=f\left(z_{\alpha \beta}\right) .
$$

This rule holds for any analytic $f(z)$ with no additional singularities at $z=z_{\alpha \beta}$; in particular, it holds for the product of shifted tree amplitudes appearing as coefficients of each pole in $z$. (Channels with only two isolated legs correspond to a collinear limit, which has a somewhat different factorization for real momenta than eq. (3.6). It turns out that for complex momenta as needed here, this case can be treated on the same footing as multiparticle factorization.)

Using this rule and summing over all residues then gives us the tree-level on-shell recursion relation,

$$
\begin{aligned}
A_{n}^{\text {tree }}\left(k_{1}, \ldots, k_{n}\right)=\sum_{\text {partitions } P} \sum_{h= \pm} A^{\text {tree }}( & \left.k_{P_{1}}, \ldots, \hat{k}_{j}, \ldots, k_{P_{-1}},-\hat{K}_{P}^{h}\right) \\
& \times \frac{i}{K_{P}^{2}} \times A^{\text {tree }}\left(k_{\bar{P}_{1}}, \ldots, \hat{k}_{l}, \ldots, k_{\bar{P}_{-1}}, \hat{K}_{P}^{-h}\right),
\end{aligned}
$$


where

$$
\begin{aligned}
& K_{P} \equiv k_{P_{1}}+k_{P_{2}}+\cdots+k_{j}+\cdots+k_{P_{-1}}, \\
& \hat{K}_{P} \equiv k_{P_{1}}+k_{P_{2}}+\cdots+\hat{k}_{j}+\cdots+k_{P_{-1}},
\end{aligned}
$$

and $h= \pm 1$ labels the helicity of the intermediate state. The hatted momenta are defined below. We have defined a partition $P$ to be a set of two or more cyclically-consecutive momentum labels containing $j$, such that the complementary set $\bar{P}$ consists of two or more cyclically-consecutive labels containing $l$,

$$
\begin{aligned}
& P \equiv\left\{P_{1}, P_{2}, \ldots, j, \ldots, P_{-1}\right\} \\
& \bar{P} \equiv\left\{\bar{P}_{1}, \bar{P}_{2}, \ldots, l, \ldots, \bar{P}_{-1}\right\} \\
& P \cup \bar{P}=\{1,2, \ldots, n\},
\end{aligned}
$$

which ensures that the sum of momenta in each partition is z-dependent. The shifted complex on-shell momenta $\hat{k}_{j}, \hat{k}_{l}$ and $\hat{K}_{P}$ in eq. (3.9) are determined by solving the on-shell condition, $\hat{K}_{P}^{2}=0$, for $z$, giving the location of the pole,

$$
z_{P}=\frac{K_{P}^{2}}{\left\langle j^{-}\left|K_{P}\right| l^{-}\right\rangle}
$$

Substituting $z \rightarrow z_{P}$ in the shifted momenta (3.2) gives,

$$
\begin{aligned}
& \hat{k}_{j}^{\mu} \equiv k_{j}\left(z_{P}\right)=k_{j}^{\mu}-\frac{1}{2} \frac{K_{P}^{2}}{\left\langle j^{-}\left|K_{P}\right| l^{-}\right\rangle}\left\langle j^{-}\left|\gamma^{\mu}\right| l^{-}\right\rangle, \\
& \hat{k}_{l}^{\mu} \equiv k_{l}\left(z_{P}\right)=k_{l}^{\mu}+\frac{1}{2} \frac{K_{P}^{2}}{\left\langle j^{-}\left|K_{P}\right| l^{-}\right\rangle}\left\langle j^{-}\left|\gamma^{\mu}\right| l^{-}\right\rangle .
\end{aligned}
$$

which define $\hat{k}_{j}$ and $\hat{k}_{l}$ appearing in the recursion relation (3.9).

At loop level, a number of new features arise. In particular, obtaining an on-shell recursion relation requires dealing with branch cuts, spurious singularities, and in some cases, the treatment of factorization using complex momenta, which can differ from 'ordinary' factorization using real momenta. We refer to channels whose complex factorization properties are non-universal, or at least not yet fully understood, as 'non-standard'. In non-standard factorization channels, double poles and unreal poles may appear in two-particle channels with like-helicity gluons $[45,46]$. Fortunately, nonstandard channels for $n$-gluon amplitudes only occur when two adjacent gluons, one of which is shifted and one of which is not, have the same helicity. As mentioned in the introduction, for the MHV amplitudes we consider here, 
our electing to shift the momenta of the two negative-helicity legs avoids all nonstandard channels.

To set up a loop-level on-shell recursion we decompose the amplitude into 'pure-cut' and 'rational' pieces,

$$
A_{n}(z)=c_{\Gamma}\left[C_{n}(z)+R_{n}(z)\right]
$$

The rational parts $R_{n}$ are defined by setting all logarithms, polylogarithms, and associated $\pi^{2}$ terms to zero,

$$
\left.\left.R_{n} \equiv \frac{1}{c_{\Gamma}} A_{n}\right|_{\mathrm{rat}} \equiv \frac{1}{c_{\Gamma}} A_{n}\right|_{\ln , \mathrm{Li}, \pi^{2} \rightarrow 0} .
$$

The pure-cut part $C_{n}$ consists of the remaining terms, all of which must contain logarithms, polylogarithms, or $\pi^{2}$ terms. The cut-containing terms have already been computed for the MHV case [18]. Our task is to obtain the rational terms (3.17) via on-shell recursion.

With the decomposition (3.16), the evaluation of a contour integral like (3.4), but for $R_{n}(z)$, is complicated, in general, by the appearance of spurious singularities. Such singularities are at kinematical points that do not correspond to any physical singularity. They arise in the course of integral reductions and often cannot be removed by algebraic means. Although the final amplitude cannot contain unphysical spurious singularities, in many cases they cancel only between (poly)logarithmic and rational terms. If we were to set up a contour integral (3.4) over the rational terms $R_{n}(z)$ instead of $A_{n}(z)$, it would pick up contributions from unphysical poles.

As a concrete example of a spurious singularity, consider the function,

$$
\frac{\ln (r)}{(1-r)^{2}}
$$

where $r$ is a ratio of momentum invariants. This function has a spurious singularity as $r \rightarrow 1$. In the full amplitude this function appears in the combination,

$$
\frac{\ln (r)+1-r}{(1-r)^{2}}
$$

which is finite as $r \rightarrow 1$.

To avoid the additional complication of spurious singularities, a good approach is to instead 'complete' the cut contribution by replacing functions like eq. (3.18) with nonsingular combinations like eq. (3.19). Although this procedure is not unique, any function that is free of unphysical spurious singularities in $z$ is satisfactory. We denote this cut 
completion by $\widehat{C}_{n}$,

$$
\widehat{C}_{n}(z)=C_{n}(z)+\widehat{C R}_{n}(z)
$$

where $\widehat{C R}_{n}(z)$ are the rational functions added in order to cancel the unphysical spurious singularities in $z$. For a given shift we do not need to remove all spurious singularities, but only those that depend on $z$. Since we have added in rational terms to the completed cuts, we should subtract them out from the remaining rational terms. Defining

$$
\widehat{R}_{n}(z)=R_{n}(z)-\widehat{C R}_{n}(z)
$$

we have,

$$
A_{n}(z)=c_{\Gamma}\left[\widehat{C}_{n}(z)+\widehat{R}_{n}(z)\right] .
$$

With the decomposition (3.22), as shown in ref. [32], the physical amplitude is given by

$$
A_{n}(0)=\operatorname{Inf} A_{n}+c_{\Gamma}\left[\widehat{C}_{n}(0)-\operatorname{Inf} \widehat{C}_{n}+R_{n}^{D}+O_{n}\right],
$$

where $\operatorname{Inf} A_{n}$ is the potential contribution to the amplitude from large $z, \widehat{C}_{n}(0)$ is the completed-cut contribution, $\operatorname{Inf} \widehat{C}_{n}$ is the potential large- $z$ spurious behavior of the completed cut, which must be subtracted off, $R_{n}^{D}$ are the recursive diagram contributions, and the 'overlap' terms $O_{n}$ remove double counting between the recursive diagrams and the rational terms that were added to complete the cuts.

Let us discuss each of the contributions to the amplitude in eq. (3.23) in turn. First consider the $\operatorname{Inf} A_{n}$ terms. As discussed in the introduction, using the known four- and fivegluon MHV amplitudes [26, 56], one can shift the two negative-helicity gluons and observe that the resulting $A_{n}(z)$ falls off as $z$ approaches infinity, that is, $\operatorname{Inf} A_{n}=0$. We assume that this property continues to hold for more than five gluons. We check this assumption after the fact, by examining various symmetries and limits of the computed amplitudes.

Now consider the $\operatorname{Inf} \widehat{C}_{n}$ subtraction. In general, since $\widehat{C}_{n}(z)$ is required only to remove unphysical singularities at finite values of $z$, it is possible that the chosen $\widehat{C}_{n}(z)$ has nonvanishing behavior as $z \rightarrow \infty$. This would introduce spurious large $z$ behavior in $\hat{R}_{n}(z)$, so we simply subtract off this large $z$ behavior with $\operatorname{Inf} \widehat{C}_{n}$. If we assume that no logarithms of $z$ appear in any surviving terms as $z \rightarrow \infty$, the procedure for computing $\operatorname{Inf} \widehat{C}_{n}$ is to start with $\widehat{C}_{n}(z)$ and series expand around $w=1 / z=0$, keeping only the $w^{0}$ term. This term is Inf $\widehat{C}_{n}$. (The assumption can easily be checked in any given case.) In many cases, including 


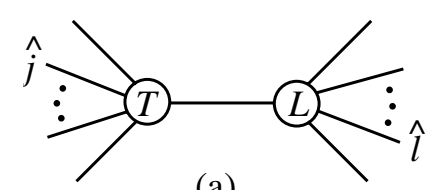

(a)

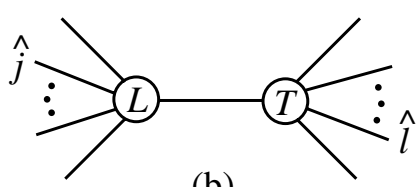

(b)

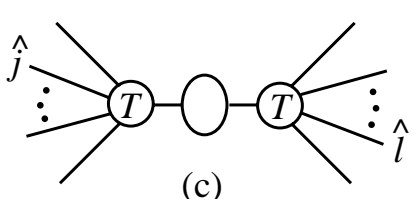

(c)

FIG. 2: Schematic representation of one-loop recursive contributions. The labels ' $T$ ' and ' $L$ ' refer to tree and loop vertices. The factorization-function contribution (c) does not appear for MHV amplitudes.

all cases in this paper, Inf $\widehat{C}_{n}$ goes to a constant at large $z$, and it can be obtained directly by taking the limit,

$$
\operatorname{Inf} \widehat{C}_{n} \equiv \lim _{z \rightarrow \infty} \widehat{C}_{n}(z) .
$$

The $R_{n}^{D}$ recursive diagram contributions on the right-hand side of eq. (3.23) are obtained via a recursion relation, following similar argumentation as at tree level. The sum over residues corresponds to a sum over factorization channels. At one loop, as depicted in fig. 2, there are in general three contributions to factorization in any given channel $s_{\alpha \ldots \beta} \rightarrow 0$,

$$
A_{L}^{\text {tree }} \times \frac{i}{s_{\alpha \cdots \beta}} \times A_{R}^{1 \text {-loop }}+A_{L}^{1 \text {-loop }} \times \frac{i}{s_{\alpha \cdots \beta}} \times A_{R}^{\text {tree }}+A_{L}^{\text {tree }} \times \frac{i \mathcal{F}^{1-\text { loop }}}{s_{\alpha \cdots \beta}} \times A_{R}^{\text {tree }} .
$$

In the first two terms, one of the factorized amplitudes is a one-loop amplitude and the other is a tree amplitude. In the $\mathcal{N}=0$ scalar loop case, the last term corresponds to a one-loop correction to the propagator [49]. Accordingly, in addition to the sum over channels, we will have a sum over these additional factorization function contributions. Taking the rational parts, we thus obtain, with the shift (3.1), the sum of recursive diagrams,

$$
\begin{aligned}
&-\sum_{\text {poles } \alpha} \operatorname{Res}_{z=z_{\alpha}} \frac{R_{n}(z)}{z} \equiv R_{n}^{D}\left(k_{1}, \ldots, k_{n}\right) \\
&=\sum_{\text {partitions } P} \sum_{h= \pm}\left\{R\left(k_{P_{1}}, \ldots, \hat{k}_{j}, \ldots, k_{P_{-1}},-\hat{K}_{P}^{h}\right)\right. \\
& \\
& \quad \times \frac{i}{K_{P}^{2}} \times A^{\text {tree }}\left(k_{\bar{P}_{1}}, \ldots, \hat{k}_{l}, \ldots, k_{\bar{P}_{-1}}, \hat{K}_{P}^{-h}\right) \\
&+A^{\text {tree }}\left(k_{P_{1}}, \ldots, \hat{k}_{j}, \ldots, k_{P_{-1}},-\hat{K}_{P}^{h}\right) \\
& \quad \times \frac{i}{K_{P}^{2}} \times R\left(k_{\bar{P}_{1}}, \ldots, \hat{k}_{l}, \ldots, k_{\bar{P}_{-1}}, \hat{K}_{P}^{-h}\right) \\
& \quad+A^{\text {tree }}\left(k_{P_{1}}, \ldots, \hat{k}_{j}, \ldots, k_{P_{-1}},-\hat{K}_{P}^{h}\right) \\
&\left.\quad \times \frac{i \mathcal{F}\left(K_{P}\right)}{K_{P}^{2}} \times A^{\text {tree }}\left(k_{\bar{P}_{1}}, \ldots, \hat{k}_{l}, \ldots, k_{\bar{P}_{-1}}, \hat{K}_{P}^{-h}\right)\right\},
\end{aligned}
$$




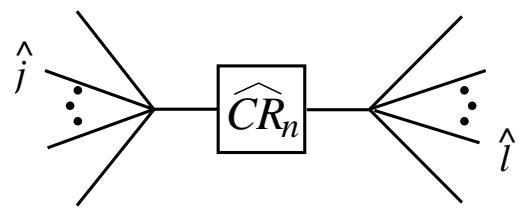

FIG. 3: Diagrammatic representation of overlap contributions. Each overlap diagram corresponds to a physical channel.

where the partition $P$ is given in eq. (3.12). The 'vertices' $R$ are one-loop amplitudes, but with all polylogarithms and $\pi^{2}$ set to vanish, as in eq. (3.17). The term containing the factorization function $\mathcal{F}$ may be found in ref. [32]. It only contributes in multi-particle channels, and only if the tree amplitude contains a pole in that channel. The MHV tree amplitudes have no multi-particle poles; hence we encounter no factorization function contributions in this paper.

The result (3.26) follows directly from the general factorization behavior of one-loop amplitudes, plus the separate factorization of pure-cut and rational terms that was established in ref. [31]. Just as in the case of the tree-level recursion (3.9) [38], it exhibits the required factorization properties in each channel $P$ (dropping the terms with logarithms, polylogarithms, and $\pi^{2}$ ). Although the $R$ functions are not complete amplitudes, they can be thought of as vertices from a diagrammatic perspective, and this equation therefore lends itself to the same kind of diagrammatic interpretation available for eq. (3.9).

Finally, consider the overlap terms $O_{n}$ in eq. (3.23). The recursive diagrams already encode the complete residues on the physical poles. Therefore, if the $\widehat{C R}_{n}$ rational terms in the completed-cuts $\widehat{C}_{n}$ have any poles in the physical channels, we must subtract these out in order to remove this double-count. Since we know $\widehat{C R}_{n}$ explicitly, it is straightforward to compute the overlap by performing the shift (3.1) and extracting the residues on the physical poles, i.e.,

$$
O_{n} \equiv \sum_{\text {poles } \alpha} \operatorname{Res}_{z=z_{\alpha}} \frac{\widehat{C R}_{n}(z)}{z}
$$

These overlap contributions may be assigned a diagrammatic interpretation, as depicted in fig. 3, with each diagram corresponding to a different physical factorization. Although the definition of the completed-cut terms $\widehat{C}_{n}$ is not unique, the ambiguity cancels between $\widehat{C}_{n}(0)$ and the overlap terms. 


\section{FIVE-, SIX-, SEVEN-, AND EIGHT-POINT AMPLITUDES}

We will now apply this formalism to the computation of $\mathcal{N}=0$ (scalar loop) amplitudes with two negative-helicity gluons. The logarithmic and polylogarithmic terms $C_{n}$ in the $\mathcal{N}=0$ case are known for all $n$-gluon amplitudes with two negative-helicity gluons. They were computed first for the case where the two negative-helicity gluons are color-adjacent [7], and more recently for the general case [18]. We quote the results for the cut parts in appendix A, including the specific cut completion we employ in this publication, eq. (A1). In appendix A we also explain why this cut completion is suitable: after performing the complex shift it contains no z-dependent spurious singularities.

The rational remainders for all-multiplicity MHV amplitudes were computed previously for the special case where the two negative-helicity gluons are adjacent [47]. Below we will first illustrate our bootstrap approach by recomputing the rational part of the five-point amplitude with two non-adjacent negative helicities [26]. This result also serves as one of the inputs for the recursive construction of amplitudes with more than five gluons. Then we will compute the remaining, previously unknown, rational terms of all $n$-gluon $\mathcal{N}=0$ amplitudes with two negative-helicity gluons in arbitrary positions. We start in this section with the computation of the six-point amplitudes. In the next section we outline the all- $n$ calculation.

Our bootstrap approach relies on previously-computed amplitudes. A list of amplitudes that enter our calculation can be found in the appendix of our companion paper [32].

\section{A. Recomputation of the five-point amplitude $A_{5 ; 1}^{\mathcal{N}=0}\left(1^{-}, 2^{+}, 3^{-}, 4^{+}, 5^{+}\right)$}

The five-point amplitude $A_{5 ; 1}^{\mathcal{N}=0}\left(1^{-}, 2^{+}, 3^{-}, 4^{+}, 5^{+}\right)$is given by [26]

$$
A_{5 ; 1}^{\mathcal{N}=0}\left(1^{-}, 2^{+}, 3^{-}, 4^{+}, 5^{+}\right)=c_{\Gamma}\left(\widehat{C}_{5}+\widehat{R}_{5}\right)
$$

where

$$
\begin{aligned}
\widehat{C}_{5}= & \frac{1}{3 c_{\Gamma}} A_{5 ; 1}^{\mathcal{N}=1}\left(1^{-}, 2^{+}, 3^{-}, 4^{+}, 5^{+}\right) \\
& +i\left[-\frac{\langle 12\rangle\langle 23\rangle\langle 34\rangle\langle 41\rangle^{2}[24]^{2}}{\langle 45\rangle\langle 51\rangle\langle 24\rangle^{2}} \frac{2 \mathrm{Ls}_{1}\left(\frac{-s_{23}}{-s_{51}}, \frac{-s_{34}}{-s_{51}}\right)+\mathrm{L}_{1}\left(\frac{-s_{23}}{-s_{51}}\right)+\mathrm{L}_{1}\left(\frac{-s_{34}}{-s_{51}}\right)}{s_{51}^{2}}\right. \\
& +\frac{\langle 32\rangle\langle 21\rangle\langle 15\rangle\langle 53\rangle^{2}[25]^{2}}{\langle 54\rangle\langle 43\rangle\langle 25\rangle^{2}} \frac{2 \mathrm{Ls}_{1}\left(\frac{-s_{12}}{-s_{34}}, \frac{-s_{51}}{-s_{34}}\right)+\mathrm{L}_{1}\left(\frac{-s_{12}}{-s_{34}}\right)+\mathrm{L}_{1}\left(\frac{-s_{51}}{-s_{34}}\right)}{s_{34}^{2}}
\end{aligned}
$$




$$
\begin{aligned}
+ & \frac{2}{3} \frac{\langle 23\rangle^{2}\langle 41\rangle^{3}[24]^{3}}{\langle 45\rangle\langle 51\rangle\langle 24\rangle} \frac{\mathrm{L}_{2}\left(\frac{-s_{23}}{-s_{51}}\right)}{s_{51}^{3}}-\frac{2}{3} \frac{\langle 21\rangle^{2}\langle 53\rangle^{3}[25]^{3}}{\langle 54\rangle\langle 43\rangle\langle 25\rangle} \frac{\mathrm{L}_{2}\left(\frac{-s_{12}}{-s_{34}}\right)}{s_{34}^{3}} \\
+ & \frac{\mathrm{L}_{2}\left(\frac{-s_{34}}{-s_{51}}\right)}{s_{51}^{3}}\left(\frac{1}{3} \frac{\langle 13\rangle[24][25](\langle 15\rangle[52]\langle 23\rangle-\langle 34\rangle[42]\langle 21\rangle)}{\langle 45\rangle}\right. \\
& \left.+\frac{2}{3} \frac{\langle 12\rangle^{2}\langle 34\rangle^{2}\langle 41\rangle[24]^{3}}{\langle 45\rangle\langle 51\rangle\langle 24\rangle}-\frac{2}{3} \frac{\langle 32\rangle^{2}\langle 15\rangle^{2}\langle 53\rangle[25]^{3}}{\langle 54\rangle\langle 43\rangle\langle 25\rangle}\right) \\
& \left.+\frac{1}{6} \frac{\langle 13\rangle^{3}(\langle 15\rangle[52]\langle 23\rangle-\langle 34\rangle[42]\langle 21\rangle)}{\langle 12\rangle\langle 23\rangle\langle 34\rangle\langle 45\rangle\langle 51\rangle} \frac{\mathrm{L}_{0}\left(\frac{-s_{34}}{-s_{51}}\right)}{s_{51}}\right], \\
\widehat{R}_{5}=\frac{2 i}{9} & \frac{\langle 13\rangle^{4}}{\langle 12\rangle\langle 23\rangle\langle 34\rangle\langle 45\rangle\langle 51\rangle}+\frac{i}{3} \frac{[24]^{2}[25]^{2}}{[12][23][34]\langle 45\rangle[51]}-\frac{i}{3} \frac{\langle 12\rangle\langle 41\rangle^{2}[24]^{3}}{\langle 45\rangle\langle 51\rangle\langle 24\rangle[23][34] s_{51}} \\
+ & \frac{i}{3} \frac{\langle 32\rangle\langle 53\rangle^{2}[25]^{3}}{\langle 54\rangle\langle 43\rangle\langle 25\rangle[21][15] s_{34}}+\frac{i}{6} \frac{\langle 13\rangle^{2}[24][25]}{s_{34}\langle 45\rangle s_{51}} .
\end{aligned}
$$

Here the contribution of an $\mathcal{N}=1$ chiral multiplet is

$$
\begin{aligned}
& A_{5 ; 1}^{\mathcal{N}=1}\left(1^{-}, 2^{+}, 3^{-}, 4^{+}, 5^{+}\right)= \\
& \begin{array}{l}
c_{\Gamma} A_{5}^{\text {tree }}\left(1^{-}, 2^{+}, 3^{-}, 4^{+}, 5^{+}\right)\left[\frac{1}{2 \epsilon}\left\{\left(\frac{\mu^{2}}{-s_{34}}\right)^{\epsilon}+\left(\frac{\mu^{2}}{-s_{51}}\right)^{\epsilon}\right\}+2\right] \\
+i c_{\Gamma}\left[\frac{\langle 13\rangle^{2}\langle 41\rangle[24]^{2}}{\langle 45\rangle\langle 51\rangle} \frac{\operatorname{Ls}_{1}\left(\frac{-s_{23}}{-s_{51}}, \frac{-s_{34}}{-s_{51}}\right)}{s_{51}^{2}}-\frac{\langle 13\rangle^{2}\langle 53\rangle[25]^{2}}{\langle 34\rangle\langle 45\rangle} \frac{\operatorname{Ls}_{1}\left(\frac{-s_{12}}{-s_{34}}, \frac{-s_{51}}{-s_{34}}\right)}{s_{34}^{2}}\right. \\
\left.\quad+\frac{1}{2} \frac{\langle 13\rangle^{3}(\langle 15\rangle[52]\langle 23\rangle-\langle 34\rangle[42]\langle 21\rangle)}{\langle 12\rangle\langle 23\rangle\langle 34\rangle\langle 45\rangle\langle 51\rangle} \frac{\mathrm{L}_{0}\left(\frac{-s_{34}}{-s_{51}}\right)}{s_{51}}\right] .
\end{array}
\end{aligned}
$$

We have introduced the function,

$$
\begin{aligned}
& \operatorname{Ls}_{1}\left(r_{1}, r_{2}\right) \equiv \frac{1}{\left(1-r_{1}-r_{2}\right)^{2}} {\left[\operatorname{Li}_{2}\left(1-r_{1}\right)+\operatorname{Li}_{2}\left(1-r_{2}\right)+\ln r_{1} \ln r_{2}-\frac{\pi^{2}}{6}\right.} \\
&\left.+\left(1-r_{1}-r_{2}\right)\left(\mathrm{L}_{0}\left(r_{1}\right)+\mathrm{L}_{0}\left(r_{2}\right)\right)\right],
\end{aligned}
$$

as well as the $\mathrm{L}_{i}$-functions,

$$
\begin{aligned}
\mathrm{L}_{0}(r) & =\frac{\ln (r)}{1-r}, \\
\mathrm{~L}_{1}(r) & =\frac{\mathrm{L}_{0}(r)+1}{1-r}, \\
\mathrm{~L}_{2}(r) & =\frac{\ln (r)-(r-1 / r) / 2}{(1-r)^{3}} .
\end{aligned}
$$

These functions are free of spurious singularities as $r \rightarrow 1$, by design.

The task of this subsection is to start with the cut completion (4.2), and use the recursive bootstrap to derive the remaining rational terms (4.3). This example has been considered 
already in ref. [31], but the detailed construction was not given there. We recall the example here because the general all-multiplicity solution will follow a similar construction. (For $n$ point amplitudes, however, we will use a cut completion which differs from the above one at $n=5$, but follows somewhat more naturally from the form of the cut parts obtained in ref. [18].)

We use a $[1,3\rangle$ shift, in the notation of eq. (3.1). The first thing we have to ensure is that the cut completion $\widehat{C}_{5}$ in eq. (4.2) is a satisfactory one, with respect to this shift. It should not produce any $z$-dependent denominator factors that vanish at spurious (unphysical) values of $z$. Notice that $\widehat{C}_{5}$ contains factors of $\langle 24\rangle$ and $\langle 25\rangle$ in denominators. These spurious singularities are not a problem for the $[1,3\rangle$ shift, because they do not acquire any dependence on $z$. (The double poles in $\langle 24\rangle$ and $\langle 25\rangle$ are actually cancelled by the behavior of the $\mathrm{Ls}_{1}$-containing functions multiplying them.) Spurious denominator factors of the form $\left(s_{23}-s_{51}\right),\left(s_{12}-s_{34}\right)$, and $\left(s_{34}-s_{51}\right)$ are dealt with via the $\mathrm{L}_{2}$ functions.

The next step is to examine the large-parameter behavior of $\widehat{C}_{5}(z)$, which can be obtained straightforwardly by applying the $[1,3\rangle$ shift to eq. (4.2). It turns out that in this case,

$$
\operatorname{Inf}_{[1,3\rangle} \widehat{C}_{5}=0
$$

The large- $z$ contributions cancel between the series expansion of the (poly)logarithmic terms and the rational terms of the cut-completion. This feature is not true in general. Specifically, the cut-completion chosen for six or more gluons, as given in appendix A, has a large- $z$ contribution (see eq. (A17)).

As mentioned in the introduction, we 'assume' that the full amplitude has vanishing large- $z$ behavior under the $[1,3\rangle$ shift,

$$
\operatorname{Inf}_{[1,3\rangle} A_{5 ; 1}^{\mathcal{N}=0}=0
$$

Of course, in the five-gluon case it is not an assumption, because we know the full amplitude in advance.

With a $[1,3\rangle$ shift, following eq. (3.26), four recursive diagrams give non-vanishing contributions, as illustrated in fig. 4 ,

$$
R_{5}^{D}=D_{5}^{(\mathrm{a})}+D_{5}^{(\mathrm{b})}+D_{5}^{(\mathrm{c})}+D_{5}^{(\mathrm{d})}
$$

As mentioned above, the necessary lower-point amplitudes and vertices are listed in the 


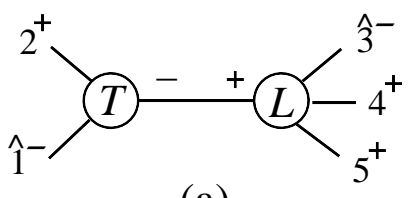

(a)

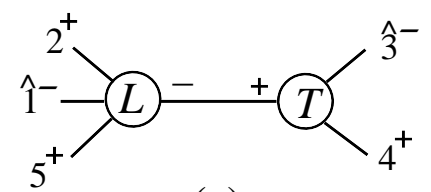

(c)

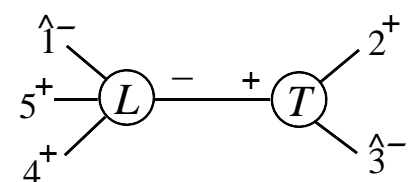

(b)

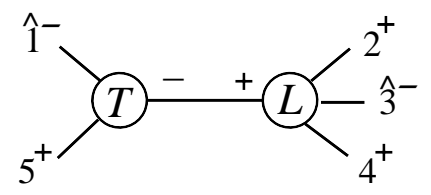

(d)

FIG. 4: Non-vanishing recursive diagrams for the amplitude $A_{5 ; 1}^{\mathcal{N}=0}\left(1^{-}, 2^{+}, 3^{-}, 4^{+}, 5^{+}\right)$, using a $[1,3\rangle$ shift, as given in eqs. (4.11)-(4.14).

appendix of our companion paper [32], except for $R_{4}\left(1^{-}, 2^{+}, 3^{-}, 4^{+}\right)$, which is given by,

$$
R_{4}\left(1^{-}, 2^{+}, 3^{-}, 4^{+}\right)=\left(\frac{1}{3 \epsilon}+\frac{8}{9}\right) A_{4}^{\text {tree }}\left(1^{-}, 2^{+}, 3^{-}, 4^{+}\right)-i \frac{\langle 13\rangle^{2}[12][23]}{\langle 34\rangle\langle 41\rangle[13]^{2}} .
$$

(The alert reader will note that $\operatorname{Inf}_{[1,3\rangle} R_{4}=i\left\langle 1^{-}|3| 2^{-}\right\rangle^{2} /\left\langle 4^{-}|1| 3^{-}\right\rangle^{2} \neq 0$, due to the last term in eq. (4.10). However, this behavior is cancelled by that of the logarithmic terms in $A_{4 ; 1}^{\mathcal{N}=0}$, so that $\operatorname{Inf}_{[1,3\rangle} A_{4 ; 1}^{\mathcal{N}=0}=0$.)

We obtain for the recursive diagrams,

$$
\begin{aligned}
D_{5}^{(\mathrm{a})} & =A_{3}^{\text {tree }}\left(-\hat{K}_{12}^{-}, \hat{1}^{-}, 2^{+}\right) \frac{i}{s_{12}} R_{4}\left(\hat{3}^{-}, 4^{+}, 5^{+} \hat{K}_{12}^{+}\right)=-\frac{i}{3} \frac{[35][24]^{3}}{\langle 45\rangle[12][13][34]^{2}}, \\
D_{5}^{(\mathrm{b})} & =R_{4}\left(\hat{1}^{-}, \hat{K}_{23}^{-}, 4^{+}, 5^{+}\right) \frac{i}{s_{23}} A_{3}^{\text {tree }}\left(\hat{3}^{-},-\hat{K}_{23}^{+}, 2^{+}\right)=i\left(\frac{1}{3 \epsilon}+\frac{8}{9}\right) \frac{\langle 13\rangle^{3}}{\langle 51\rangle\langle 23\rangle\langle 24\rangle\langle 45\rangle}, \\
D_{5}^{(\mathrm{c})} & =R_{4}\left(\hat{1}^{-}, 2^{+}, \hat{K}_{34}^{-}, 5^{+}\right) \frac{i}{s_{34}} A_{3}^{\text {tree }}\left(\hat{3}^{-}, 4^{+},-\hat{K}_{34}^{+}\right) \\
& =i\left(\frac{1}{3 \epsilon}+\frac{8}{9}\right) \frac{\langle 13\rangle^{3}\langle 14\rangle}{\langle 51\rangle\langle 12\rangle\langle 34\rangle\langle 45\rangle\langle 24\rangle}+i \frac{\langle 13\rangle^{3}}{\langle 14\rangle\langle 34\rangle\langle 25\rangle^{2}}, \\
D_{5}^{(\mathrm{d})} & =A_{3}^{\text {tree }}\left(\hat{1}^{-},-\hat{K}_{51}^{-}, 5^{+}\right) \frac{i}{s_{51}} R_{4}\left(\hat{3}^{-}, 4^{+}, \hat{K}_{51}^{+}, 2^{+}\right)=\frac{i}{3} \frac{[35]^{3}[24]^{3}}{\langle 24\rangle[13][23]^{2}[34]^{2}[15]} .
\end{aligned}
$$

As remarked earlier, there is no factorization-function contribution for the case of MHV amplitudes.

In addition, there are four channels that can potentially contribute overlap terms,

$$
\begin{aligned}
z^{(\mathrm{a})}=-\frac{[12]}{[23]}, & z^{(\mathrm{b})}=\frac{\langle 23\rangle}{\langle 12\rangle}, \\
z^{(\mathrm{c})}=-\frac{\langle 34\rangle}{\langle 14\rangle}, & z^{(\mathrm{d})}=\frac{[15]}{[35]} .
\end{aligned}
$$




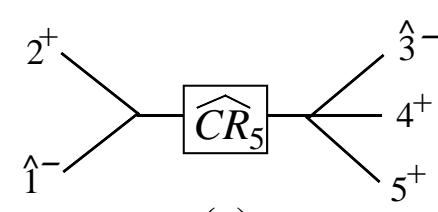

(a)

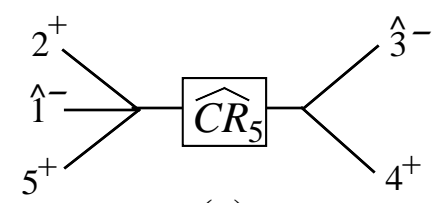

(c)

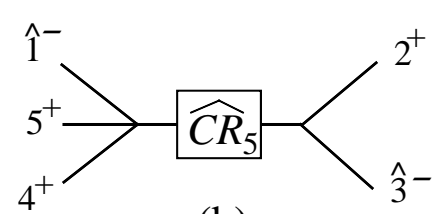

(b)

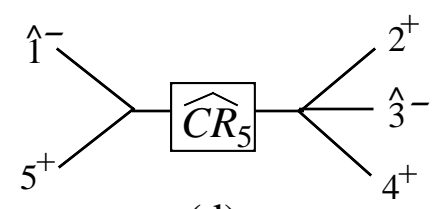

(d)

FIG. 5: Channels giving overlap contributions to $A_{5 ; 1}^{\mathcal{N}=0}\left(1^{-}, 2^{+}, 3^{-}, 4^{+}, 5^{+}\right)$with a $[1,3\rangle$ shift, at the values of $z$ given in eq. (4.15).

The overlap contributions are illustrated in fig. 5. For our choice of cut completion, eq. (4.2), they are given by,

$$
\begin{aligned}
O^{(\mathrm{a})}= & -\frac{i}{3} \frac{\langle 12\rangle[24]^{3}}{[12][34]^{2}\langle 45\rangle\langle 25\rangle}, \\
O^{(\mathrm{b})}= & -\frac{i}{3}\left(\frac{1}{\epsilon}+2\right) \frac{\langle 13\rangle^{3}}{\langle 51\rangle\langle 23\rangle\langle 24\rangle\langle 45\rangle}, \\
O^{(\mathrm{c})}= & -\frac{i}{3}\left(\frac{1}{\epsilon}+2\right) \frac{\langle 13\rangle^{3}\langle 14\rangle}{\langle 51\rangle\langle 12\rangle\langle 34\rangle\langle 45\rangle\langle 24\rangle}+\frac{i}{6} \frac{\langle 13\rangle^{2}[24]\langle 14\rangle}{s_{34}\langle 45\rangle\langle 15\rangle\langle 24\rangle}-i \frac{4}{3} \frac{\langle 13\rangle^{2}[25]\langle 15\rangle}{s_{34}\langle 45\rangle\langle 14\rangle\langle 25\rangle} \\
& -i \frac{2}{3} \frac{\langle 13\rangle^{2}[45]}{s_{34}\langle 24\rangle\langle 25\rangle}+\frac{i}{3} \frac{\langle 13\rangle^{3}\left\langle 4^{-}|(3-5)| 2^{-}\right\rangle}{s_{34}\langle 34\rangle\langle 45\rangle\langle 14\rangle\langle 25\rangle}-i \frac{\langle 12\rangle^{2}\langle 13\rangle^{3}[23]\langle 45\rangle}{s_{34}\langle 15\rangle\langle 14\rangle^{2}\langle 24\rangle\langle 25\rangle^{2}} \\
& +2 i \frac{\langle 13\rangle^{2}\langle 15\rangle^{2}\langle 24\rangle[25]}{s_{34}\langle 45\rangle\langle 14\rangle^{2}\langle 25\rangle^{2}}-i \frac{\langle 12\rangle\langle 13\rangle^{2}[25](\langle 12\rangle\langle 45\rangle-\langle 15\rangle\langle 24\rangle)}{s_{34}\langle 14\rangle^{2}\langle 24\rangle\langle 25\rangle^{2}} \\
& -i \frac{\langle 13\rangle^{3}\langle 15\rangle[35]}{s_{34}\langle 14\rangle^{2}\langle 25\rangle^{2}}-i \frac{\langle 13\rangle^{3}}{\langle 15\rangle\langle 24\rangle\langle 34\rangle\langle 25\rangle}, \\
O^{(\mathrm{d})}= & \frac{i}{6} \frac{\langle 13\rangle[24][35]\left\langle 1^{-}|(2-5)| 4^{-}\right\rangle}{[34]^{2}\langle 45\rangle\langle 15\rangle[15]\langle 24\rangle}-\frac{i}{3} \frac{\langle 14\rangle^{2}\langle 12\rangle[24]^{3}}{[23][34]\langle 45\rangle[15]\langle 15\rangle^{2}\langle 24\rangle} \\
& +\frac{i}{3} \frac{\langle 14\rangle[24]^{3}[35]^{2}}{[23]^{2}[34]^{2}\langle 45\rangle[15]\langle 24\rangle}+\frac{i}{3} \frac{\langle 15\rangle[45]^{2}\left(s_{15}+s_{35}\right)}{[34]^{2}\langle 45\rangle[15]\langle 24\rangle\langle 25\rangle}
\end{aligned}
$$

The final result is the sum of all contributions, eqs. (4.7), (4.11)-(4.14), and (4.16)-(4.19), $\widehat{R}_{5}\left(1^{-}, 2^{+}, 3^{-}, 4^{+}, 5^{+}\right)=-\operatorname{Inf}_{[1,3\rangle} \widehat{C}_{5}+D_{5}{ }^{(\mathrm{a})}+D_{5}{ }^{(\mathrm{b})}+D_{5}{ }^{(\mathrm{c})}+D_{5}{ }^{(\mathrm{d})}+O_{5}^{(\mathrm{a})}+O_{5}^{(\mathrm{b})}+O_{5}^{(\mathrm{c})}+O_{5}^{(\mathrm{d})}$.

The result agrees with eq. (4.3), after appropriate simplification. The complete rational part $R_{5}\left(1^{-}, 2^{+}, 3^{-}, 4^{+}, 5^{+}\right)$, which is needed in the recursive construction of higher-point 
amplitudes in the next subsection, is found by adding the rational terms of $\widehat{C}_{5}$ and $\widehat{R}_{5}$. It can be read off from eqs. (4.2) and (4.3),

$$
R_{5}\left(1^{-}, 2^{+}, 3^{-}, 4^{+}, 5^{+}\right)=\left.\frac{1}{c_{\Gamma}} A_{5 ; 1}^{\mathcal{N}=0}\left(1^{-}, 2^{+}, 3^{-}, 4^{+}, 5^{+}\right)\right|_{\ln , \mathrm{Li}_{2}, \pi^{2}=0}
$$

\section{B. Six-point amplitudes with two negative helicities}

Without loss of generality, we label one of the negative-helicity gluons as gluon 1 and the other as gluon $m$. Other configurations can be obtained by cyclicly permuting the labels of the gluons. We introduce a shorthand for the argument list in the $n$-gluon case,

$$
A_{n ; 1}^{\mathcal{N}=0}(1, m) \equiv A_{n ; 1}^{\mathcal{N}=0}\left(1^{-}, 2^{+}, \ldots,(m-1)^{+}, m^{-},(m+1)^{+}, \ldots, n^{+}\right)
$$

The amplitude where the other negative-helicity gluon is adjacent, $A_{n ; 1}^{\mathcal{N}=0}(1,2)$, can be found in refs. [31, 47]. At six points, there are two other independent configurations with two negative-helicity gluons, $A_{6 ; 1}^{\mathcal{N}=0}(1,3)$ and $A_{6 ; 1}^{\mathcal{N}=0}(1,4)$. We choose a $[1, m\rangle$ shift, in the notation of eq. (3.1), with $m=3,4$ in the respective cases.

The cut parts were obtained in ref. [18]. As explained above, we complete these cutcontaining terms to remove $z$-dependent spurious singularities that arise when performing the $[1, m\rangle$ shift we've chosen. The result is given in eq. (A1). Taking $n=6$ in this expression gives the completed-cut parts required here, $\widehat{C}_{6}(0) \equiv \widehat{C}_{6}(1, m)$. It is straightforward to compute $\operatorname{Inf} \widehat{C}_{6}(1, m)$ by first shifting $\widehat{C}_{6}(0)$ by the $[1, m\rangle$ shift, to obtain $\widehat{C}_{6}(z)$, and then series expanding around $w=1 / z$, keeping the $w^{0}$ term. (The result is given by eq. (A17), with $n=6$.)

For the case of $A_{6 ; 1}^{\mathcal{N}=0}(1,3)$ with a $[1,3\rangle$ shift, the recursive contribution is given by,

$$
\begin{aligned}
R_{6}^{D}(1,3) & =A_{3}^{\text {tree }}\left(-\hat{K}_{12}^{-}, \hat{1}^{-}, 2^{+}\right) \frac{i}{s_{12}} R_{5}\left(\hat{3}^{-}, 4^{+}, 5^{+}, 6^{+}, \hat{K}_{12}^{+}\right) \\
& +R_{5}\left(\hat{1}^{-}, \hat{K}_{23}^{-}, 4^{+}, 5^{+}, 6^{+}\right) \frac{i}{s_{23}} A_{3}^{\text {tree }}\left(\hat{3}^{-},-\hat{K}_{23}^{+}, 2^{+}\right) \\
& +A_{4}^{\text {tree }}\left(\hat{1}^{-}, \hat{K}_{234}^{-}, 5^{+}, 6^{+}\right) \frac{i}{s_{234}} R_{4}\left(\hat{3}^{-}, 4^{+},-\hat{K}_{234}^{+}, 2^{+}\right) \\
& +R_{4}\left(\hat{1}^{-}, \hat{K}_{234}^{+}, 5^{+}, 6^{+}\right) \frac{i}{s_{234}} A_{4}^{\text {tree }}\left(\hat{3}^{-}, 4^{+},-\hat{K}_{234}^{-}, 2^{+}\right) \\
& +R_{5}\left(\hat{1}^{-}, 2^{+}, \hat{K}_{34}^{-}, 5^{+}, 6^{+}\right) \frac{i}{s_{34}} A_{3}^{\text {tree }}\left(\hat{3}^{-}, 4^{+},-\hat{K}_{34}^{+}\right) \\
& +A_{4}^{\text {tree }}\left(\hat{1}^{-}, 2^{+}, \hat{K}_{345}^{-}, 6^{+}\right) \frac{i}{s_{345}} R_{4}\left(\hat{3}^{-}, 4^{+}, 5^{+},-\hat{K}_{345}^{+}\right)
\end{aligned}
$$




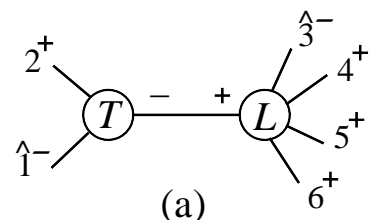

(a)

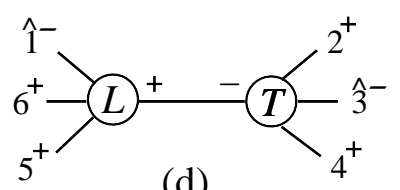

(d)

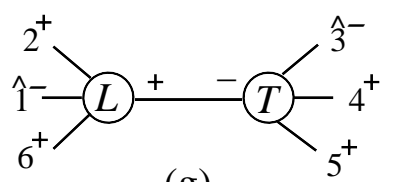

$(\mathrm{g})$

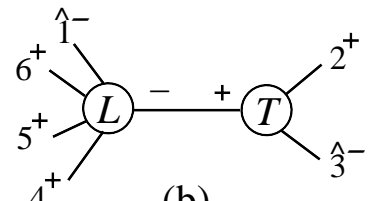

(b)

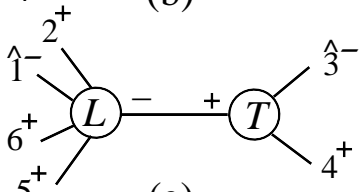

(e)

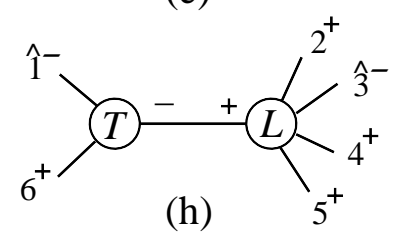

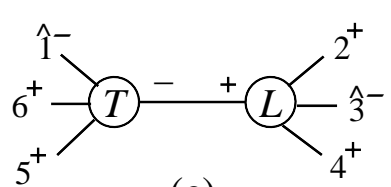

(c)

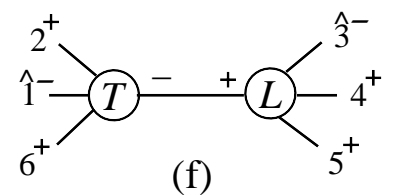

(f)

FIG. 6: Non-vanishing recursive diagrams for the amplitude $A_{6 ; 1}^{\mathcal{N}=0}(1,3)$, using a $[1,3\rangle$ shift, as given in eq. (4.23).

$$
\begin{aligned}
& +R_{4}\left(\hat{1}^{-}, 2^{+}, \hat{K}_{345}^{+}, 6^{+}\right) \frac{i}{s_{345}} A_{4}^{\text {tree }}\left(\hat{3}^{-}, 4^{+}, 5^{+},-\hat{K}_{345}^{-}\right) \\
& +A_{3}^{\text {tree }}\left(\hat{1}^{-},-\hat{K}_{61}^{-}, 6^{+}\right) \frac{i}{s_{61}} R_{5}\left(\hat{3}^{-}, 4^{+}, 5^{+}, \hat{K}_{61}^{+}, 2^{+}\right)
\end{aligned}
$$

where we have omitted terms that vanish. As mentioned in section III, there is no factorization-function contribution for MHV amplitudes. Equation (4.23) is illustrated in fig. 6. The amplitudes entering eq. (4.23) are listed in the appendix of ref. [32] and in section IV A. Note that the lower-point amplitudes have at most two negative helicities. This general feature of the one-loop MHV amplitudes allows us to solve the recursion relation for all $n$, as shown in section $\mathrm{V}$.

Once a cut completion is chosen, we can apply the shift $[1,3\rangle$ to $\widehat{C R}_{6}$ to obtain the overlap contributions $O_{6}$. For $A_{6 ; 1}^{\mathcal{N}=0}(1,3)$ there are six possible channels which can give poles, illustrated in fig. 7 ,

$$
\begin{aligned}
z^{(\mathrm{a})}=-\frac{[12]}{[23]}, & z^{(\mathrm{b})}=\frac{\langle 23\rangle}{\langle 12\rangle}, \\
z^{(\mathrm{c})}=-\frac{s_{234}}{\left\langle 1^{-}|(2+4)| 3^{-}\right\rangle}, & z^{(\mathrm{d})}=-\frac{\langle 34\rangle}{\langle 14\rangle}, \\
z^{(\mathrm{e})}=-\frac{s_{345}}{\left\langle 1^{-}|(4+5)| 3^{-}\right\rangle}, & z^{(\mathrm{f})}=\frac{[16]}{[36]} .
\end{aligned}
$$

Depending on the specific cut completion, some channels may give vanishing overlap contributions. 

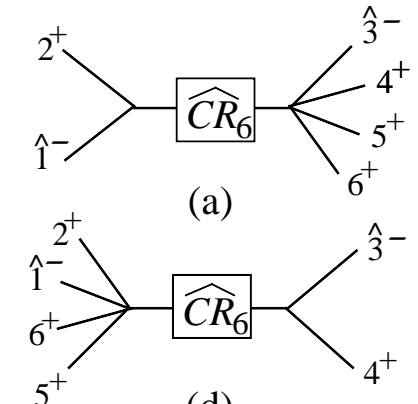

(d)

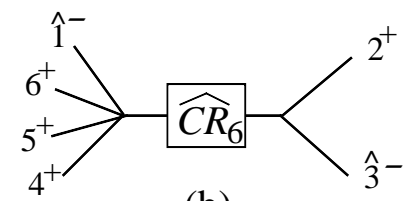

(b)

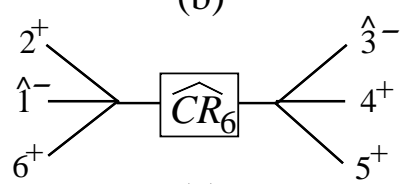

(e)

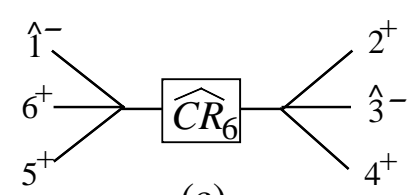

(c)

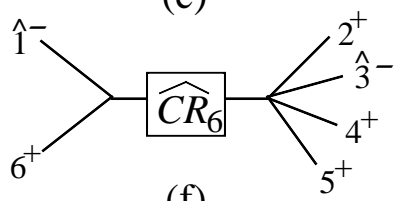

(f)

FIG. 7: Channels that can give overlap contributions to $A_{6 ; 1}^{\mathcal{N}=0}(1,3)$ with a $[1,3\rangle$ shift, at the values of $z$ given in eq. (4.24).

After assembling and simplifying the recursive and overlap diagrams, we obtain the result presented in eq. (B2) of appendix B for $\widehat{R}_{6}(1,3)$, for the specific cut completion (A1). We have checked numerically that our result is symmetric under the flip (123456) $\leftrightarrow(321654)$, even though the recursion relation (4.23) does not keep the flip symmetry manifest. Furthermore, our result displays the correct multi-particle and collinear factorization limits, as well as cancellation of all spurious singularities. We give some numerical values in section IV C.

The computation of $A_{6 ; 1}^{\mathcal{N}=0}(1,4)$ uses the $[1,4\rangle$ shift. The recursive contribution is given by,

$$
\begin{aligned}
R_{6}^{D}(1,4) & =A_{3}^{\text {tree }}\left(\hat{1}^{-}, 2^{+},-\hat{K}_{12}^{-}\right) \frac{i}{s_{12}} R_{5}\left(\hat{4}^{-}, 5^{+}, 6^{+}, \hat{K}_{12}^{+}, 3^{+}\right) \\
& +A_{4}^{\text {tree }}\left(\hat{1}^{-}, \hat{K}_{234}^{-}, 5^{+}, 6^{+}\right) \frac{i}{s_{234}} R_{4}\left(\hat{4}^{-},-\hat{K}_{234}^{+}, 2^{+}, 3^{+}\right) \\
& +R_{4}\left(\hat{1}^{-}, \hat{K}_{234}^{+}, 5^{+}, 6^{+}\right) \frac{i}{s_{234}} A_{4}^{\text {tree }}\left(\hat{4}^{-},-\hat{K}_{234}^{-}, 2^{+}, 3^{+}\right) \\
& +R_{5}\left(\hat{1}^{-}, 2^{+}, \hat{K}_{34}^{-}, 5^{+}, 6^{+}\right) \frac{i}{s_{34}} A_{3}^{\text {tree }}\left(\hat{4}^{-},-\hat{K}_{34}^{+}, 3^{+}\right) \\
& +A_{4}^{\text {tree }}\left(\hat{1}^{-}, 2^{+}, \hat{K}_{345}^{-}, 6^{+}\right) \frac{i}{s_{345}} R_{4}\left(\hat{4}^{-}, 5^{+},-\hat{K}_{345}^{+}, 3^{+}\right) \\
& +R_{4}\left(\hat{1}^{-}, 2^{+}, \hat{K}_{345}^{+}, 6^{+}\right) \frac{i}{s_{345}} A_{4}^{\text {tree }}\left(\hat{4}^{-}, 5^{+},-\hat{K}_{345}^{-}, 3^{+}\right) \\
& +R_{5}\left(\hat{1}^{-}, 2^{+}, 3^{+}, \hat{K}_{45}^{-}, 6^{+}\right) \frac{i}{s_{45}} A_{3}^{\text {tree }}\left(\hat{4}^{-}, 5^{+},-\hat{K}_{45}^{+}\right) \\
& +A_{4}^{\text {tree }}\left(\hat{1}^{-}, 2^{+}, 3^{+}, \hat{K}_{456}^{-}\right) \frac{i}{s_{456}} R_{4}\left(\hat{4}^{-}, 5^{+}, 6^{+},-\hat{K}_{456}^{+}\right) \\
& +R_{4}\left(\hat{1}^{-}, 2^{+}, 3^{+}, \hat{K}_{456}^{+}\right) \frac{i}{s_{456}} A_{4}^{\text {tree }}\left(\hat{4}^{-}, 5^{+}, 6^{+},-\hat{K}_{456}^{-}\right) \\
& +A_{3}^{\text {tree }}\left(\hat{1}^{-},-\hat{K}_{61}^{-}, 6^{+}\right) \frac{i}{s_{61}} R_{5}\left(\hat{4}^{-}, 5^{+}, \hat{K}_{61}^{+}, 2^{+}, 3^{+}\right) \\
&
\end{aligned}
$$




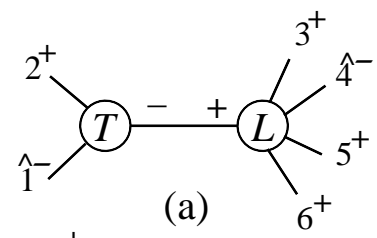

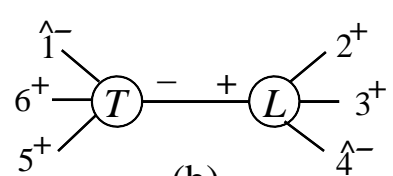

(b)

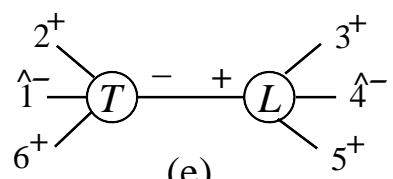

(e)

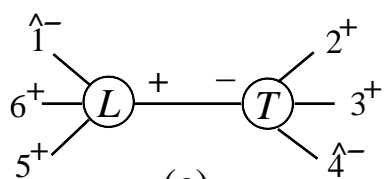

(c)

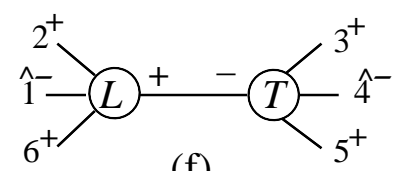

(f)

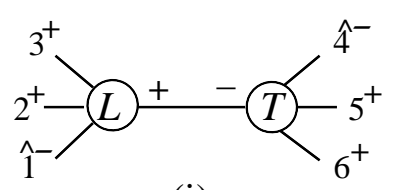

(i) (h)

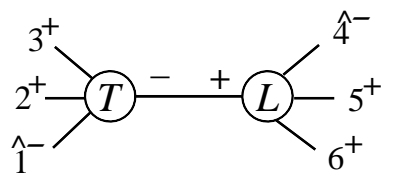

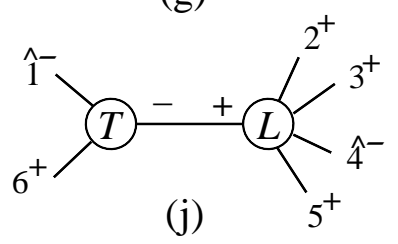

(j)

FIG. 8: Non-vanishing recursive diagrams for the amplitude $A_{6 ; 1}^{\mathcal{N}}=0(1,4)$, using a $[1,4\rangle$ shift, as given in eq. (4.25).

as illustrated in fig. 8. Note that the amplitude $A_{6 ; 1}^{\mathcal{N}=0}(1,4)$ has a flip symmetry under the simultaneous exchange of gluons $2 \leftrightarrow 6$ and $3 \leftrightarrow 5$. This flip symmetry remains manifest in the presence of the $[1,4\rangle$ shift. (A second flip symmetry, (123456) $\leftrightarrow(432165)$, is not kept manifest.) Therefore we only need to compute six recursive diagrams in eq. (4.25); the other four diagrams can be obtained by the flip symmetry. Diagram (a) can be obtained from diagram (j) by the flip symmetry, likewise diagram (b) from (h), (c) from (i), and (d) from (g). Diagrams (e) and (f) are invariant under the symmetry.

With a $[1,4\rangle$ shift there are seven channels that can give contributions to the overlap $\mathrm{O}_{6}(1,4)$ as illustrated in fig. 9,

$$
\begin{aligned}
z^{(\mathrm{a})}=-\frac{[12]}{[24]}, & z^{(\mathrm{b})}=-\frac{s_{234}}{\left\langle 1^{-}|(2+3)| 4^{-}\right\rangle}, \\
z^{(\mathrm{c})}=\frac{\langle 34\rangle}{\langle 13\rangle}, & z^{(\mathrm{d})}=-\frac{s_{345}}{\left\langle 1^{-}|(3+5)| 4^{-}\right\rangle}, \\
z^{(\mathrm{e})}=-\frac{\langle 45\rangle}{\langle 15\rangle}, & z^{(\mathrm{f})}=-\frac{s_{456}}{\left\langle 1^{-}|(5+6)| 4^{-}\right\rangle}, \\
z^{(\mathrm{g})}=\frac{[16]}{[46]} &
\end{aligned}
$$




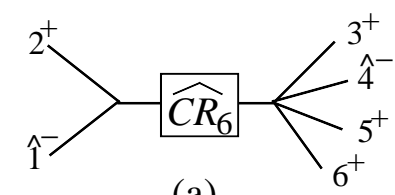

(a)

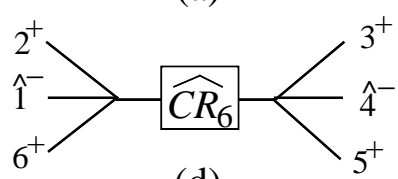

(d)

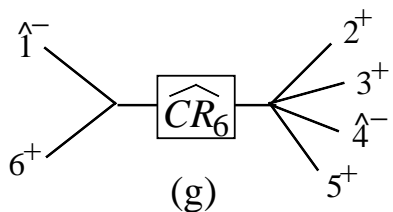

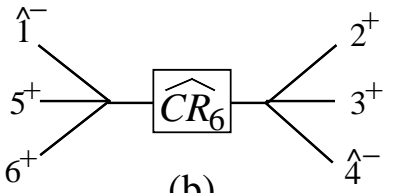

(b)

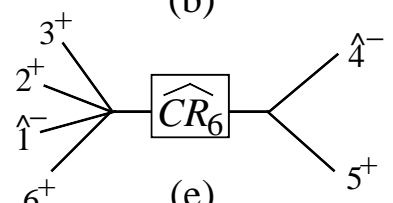

(e)

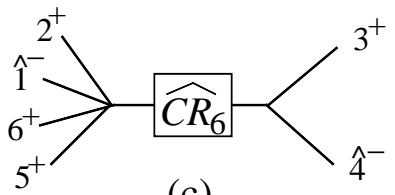

(c)

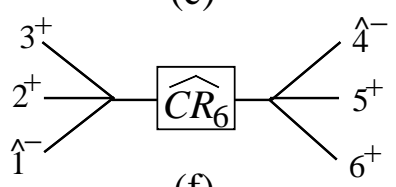

(f)

FIG. 9: Channels giving overlap contributions to $A_{6 ; 1}^{\mathcal{N}=0}(1,4)$ with a $[1,4\rangle$ shift, at the values of $z$ given in eq. (4.26).

Again, we can make use of the aforementioned flip symmetry, $2 \leftrightarrow 6$ and $3 \leftrightarrow 5$, because our cut completion, $\widehat{C R}_{6}(1,4)$ in eq. (A13), was chosen to be flip symmetric. (This property relies in part on the symmetry and antisymmetry under $r \rightarrow 1 / r$ of the functions $\mathrm{L}_{2}(r)$ and $\widehat{\mathrm{L}}_{1}(r)$ defined in eqs. (4.6) and (A10), respectively.) The flip symmetry relates overlaps $O^{(\mathrm{a})}$ and $O^{(\mathrm{g})}, O^{(\mathrm{b})}$ and $O^{(\mathrm{f})}$, and $O^{(\mathrm{c})}$ and $O^{(\mathrm{e})}$, whereas $O^{(\mathrm{d})}$ is invariant.

The result for the rational part $\widehat{R}_{6}(1,4)$, complementing the completed-cut terms given in eq. (A1), is listed in appendix B. We have checked numerically that the other, nonmanifest, flip symmetry $(123456) \leftrightarrow(432165)$ of this amplitude holds, and that the proper factorization limits are obtained in all channels. We give numerical results for the amplitude in the next subsection.

\section{Numerical results for various six-, seven- and eight-point amplitudes}

All- $n$ analytical results were previously found for the $\mathcal{N}=0$ one-loop amplitudes with all gluons of positive helicity [58], and all but one gluon of positive helicity [59]. These results have been recomputed via a simplified version of the bootstrap approach [45, 46]. (It is simpler because these amplitudes contain no cuts.) The full bootstrap, as described in section III, has been used to compute the $n$-gluon split-helicity next-to-MHV amplitudes [32], using cut-containing terms from ref. [29]. As described in more detail in the next section, we 
have now obtained all- $n$ results for all MHV configurations, by combining the cut parts [18] with the rational terms from section $\mathrm{V}$ of this paper. The case of MHV amplitudes with two nearest neighboring negative helicity legs in the color ordering was worked out in ref. [47].

Here we list some numerical values of the MHV amplitudes for a particular phase-space point, defined below, for six, seven, and eight gluons. In the six-point case, we compare to results [4] that were obtained with a semi-numerical approach [3], and more recently, analytically [52]. For the seven- and eight-gluon cases, we have checked numerically that our results have the correct flip symmetries (in fact this was checked up to $n=11$ ), as well the correct limits in all multi-particle and collinear factorization channels.

Specifically, for the six-point case we use the same phase-space point as in refs. [4, 32], with the six momenta $k_{i}$ chosen as follows,

$$
\begin{aligned}
& k_{1}=\frac{\mu}{2}(-1,+\sin \theta,+\cos \theta \sin \phi,+\cos \theta \cos \phi), \\
& k_{2}=\frac{\mu}{2}(-1,-\sin \theta,-\cos \theta \sin \phi,-\cos \theta \cos \phi), \\
& k_{3}=\frac{\mu}{3}(1,1,0,0), \\
& k_{4}=\frac{\mu}{7}(1, \cos \beta, \sin \beta, 0), \\
& k_{5}=\frac{\mu}{6}(1, \cos \alpha \cos \beta, \cos \alpha \sin \beta, \sin \alpha), \\
& k_{6}=-k_{1}-k_{2}-k_{3}-k_{4}-k_{5},
\end{aligned}
$$

where

$$
\theta=\frac{\pi}{4}, \quad \phi=\frac{\pi}{6}, \quad \alpha=\frac{\pi}{3}, \quad \cos \beta=-\frac{7}{19} .
$$

Note that the energies of $k_{1}$ and $k_{2}$ are negative and $k_{i}^{2}=0$. In order to have energies of $O(1)$, the authors of ref. [4] chose the scale $\mu=n=6[\mathrm{GeV}]$. As usual $\mu$ also denotes the scale which is used to carry the dimensionality of the $D$-dimensional integrals.

For the seven-point case we choose the same kinematic point as in ref. [32],

$$
\begin{aligned}
k_{1} & =\frac{\mu}{2}(-1,+\sin \theta,+\cos \theta \sin \phi,+\cos \theta \cos \phi), \\
k_{2} & =\frac{\mu}{2}(-1,-\sin \theta,-\cos \theta \sin \phi,-\cos \theta \cos \phi), \\
k_{3} & =\frac{\mu}{3}(1,1,0,0), \\
k_{4} & =\frac{\mu}{8}(1, \cos \beta, \sin \beta, 0), \\
k_{5} & =\frac{\mu}{10}(1, \cos \alpha \cos \beta, \cos \alpha \sin \beta, \sin \alpha),
\end{aligned}
$$


TABLE I: Numerical results for the non-vanishing $\mathcal{N}=4$ six-, seven-, and eight-point amplitudes with two negative-helicity legs in the FDH scheme. The kinematic points are given in eqs. (4.27), (4.29) and (4.31). The analytic expressions used for this table are from refs. [6].

\begin{tabular}{||c||c|c||}
\hline \hline Helicity & $1 / \epsilon$ & $\epsilon^{0}$ \\
\hline \hline--++++ & $448.1350970+i 288.8591589$ & $231.6837670+i 1219.687214$ \\
\hline-+-+++ & $145.1068197+i 93.53303095$ & $75.01955289+i 394.9365577$ \\
\hline-++-++ & $7.064109769-i 10.20934744$ & $28.56235909-i 4.462571113$ \\
\hline \hline--+++++ & $2923.502435+i 683.4723607$ & $5112.775012+i 6035.881921$ \\
\hline-+-++++ & $946.6344939+i 221.3093804$ & $1655.524253+i 1954.427662$ \\
\hline-++-+++ & $21.02279700-i 56.55151527$ & $133.2644743-i 86.65176251$ \\
\hline \hline--++++++ & $62.40652480+i 54.16810878$ & $42.55501770+i 181.2843342$ \\
\hline-+-+++++ & $7.274637638+i 6.314297490$ & $4.960576389+i 21.13205060$ \\
\hline-++-++++ & $0.06051809107+i 0.01883973611$ & $0.08570866540+i 0.1142513680$ \\
\hline-+++-+++ & $1.592056562-i 1.878665237$ & $5.386800498-i 1.331984344$ \\
\hline \hline
\end{tabular}

$$
\begin{aligned}
& k_{6}=\frac{\mu}{12}(1, \cos \beta \cos \gamma, \sin \beta \cos \gamma, \sin \gamma), \\
& k_{7}=-k_{1}-k_{2}-k_{3}-k_{4}-k_{5}-k_{6},
\end{aligned}
$$

where

$$
\theta=\frac{\pi}{4}, \quad \phi=\frac{\pi}{6}, \quad \alpha=\frac{\pi}{3}, \quad \gamma=\frac{2 \pi}{3}, \quad \cos \beta=-\frac{37}{128},
$$

and $\mu=n=7 \mathrm{GeV}$.

At eight points, it turns out that the choice in ref. [32] sits on top of a possible spurious singularity (where $\left\langle 7^{ \pm}|(1+2)| 5^{ \pm}\right\rangle=0$ ), so here we choose a different reference kinematic point,

$$
\begin{aligned}
k_{1} & =\frac{\mu}{2}(-1,1 / \sqrt{2},-1 / 2,1 / 2), \\
k_{2} & =\frac{\mu}{2}(-1,-1 / \sqrt{2}, 1 / 2,-1 / 2), \\
k_{3} & =\frac{\mu}{5}(1,1,0,0), \\
k_{4} & =\frac{\mu}{5}(1, \cos \beta, \sin \beta, 0), \\
k_{5} & =\frac{\mu}{6}(1, \cos \alpha \cos \beta, \cos \alpha \sin \beta, \sin \alpha),
\end{aligned}
$$


TABLE II: Numerical results for the non-vanishing $\mathcal{N}=1$ chiral contributions to six-, seven-, and eight-point MHV amplitudes. The kinematic points are given in eqs. (4.27), (4.29), and (4.31). The analytical expressions were obtained from ref. [7].

\begin{tabular}{||c||c|c||}
\hline \hline Helicity & $1 / \epsilon$ & $\epsilon^{0}$ \\
\hline \hline--++++ & $-28.11035867+i 4.643367883$ & $-108.9419206+i 35.02980993$ \\
\hline-+-+++ & $-9.102176499+i 1.503529518$ & $-35.86914908+i 5.750500896$ \\
\hline-++-++ & $0.08664490662+i 0.6577514371$ & $-2.226022769+i 3.230760457$ \\
\hline \hline--+++++ & $-104.5611840+i 45.34709475$ & $-429.6932951+i 209.0560823$ \\
\hline-+-++++ & $-33.85706894+i 14.68345762$ & $-162.2581945+i 46.94912355$ \\
\hline-++-+++ & $0.6394298622+i 2.199205804$ & $-9.326523676+i 12.89705727$ \\
\hline \hline--++++++ & $-3.088708769+i 0.2144746165$ & $-7.455275518+i 0.7776396411$ \\
\hline-+-+++++ & $-0.3600462794+i 0.02500099345$ & $-0.7753751687-i 0.06138368438$ \\
\hline-++-++++ & $-0.002103801109+i 0.001101616598$ & $-0.008916187143-i 0.003055492662$ \\
\hline-+++-+++ & $0.007480422983+i 0.09196001806$ & $-0.2405797542+i 0.3716908859$ \\
\hline \hline
\end{tabular}

TABLE III: Numerical results for six-, seven-, and eight-point $\mathcal{N}=0$ amplitudes as described in the text, evaluated at the specific phase space points in eqs. (4.27), (4.29), and (4.31).

\begin{tabular}{||c||c|c||}
\hline \hline Helicity & $1 / \epsilon$ & $\epsilon^{0}$ \\
\hline \hline--++++ & $-9.370119558+i 1.547789294$ & $-45.80779561+i 13.03695870$ \\
\hline-+-+++ & $-3.034058833+i 0.5011765059$ & $-15.20562226+i 1.709378044$ \\
\hline-++-++ & $0.02888163554+i 0.2192504790$ & $-1.449837907+i 0.1763294054$ \\
\hline \hline--+++++ & $-34.85372799+i 15.11569825$ & $-176.2169235+i 87.93931019$ \\
\hline-+-++++ & $-11.28568965+i 4.894485872$ & $-58.27730664+i 13.67438826$ \\
\hline-++-+++ & $0.2131432874+i 0.7330686014$ & $-6.238940131-i 0.4283898751$ \\
\hline \hline--++++++ & $-1.029569590+i 0.07149153884$ & $-4.244770988+i 0.3284878412$ \\
\hline-+-+++++ & $-0.1200154265+i 0.008333664483$ & $-0.5328645055-i 0.1225617739$ \\
\hline-++-++++ & $-0.0007012670363+i 0.0003672055326$ & $0.007966818918-i 0.05081471136$ \\
\hline-+++-+++ & $0.002493474328+i 0.03065333935$ & $-0.1173717691+i 0.1867703328$ \\
\hline \hline
\end{tabular}




$$
\begin{aligned}
& k_{6}=\frac{\mu}{7}(1, \cos \gamma \cos \beta, \cos \gamma \sin \beta, \sin \gamma), \\
& k_{7}=\frac{\mu}{8}(1, \cos \delta \cos \beta, \cos \delta \sin \beta, \sin \delta), \\
& k_{8}=-k_{1}-k_{2}-k_{3}-k_{4}-k_{5}-k_{6}-k_{7},
\end{aligned}
$$

where

$$
\cos \alpha=\frac{8}{17}, \quad \cos \beta=-\frac{7193}{8258}, \quad \cos \delta=-\frac{3}{5}, \quad \cos \gamma=\frac{3}{5}
$$

and $\mu=n=8 \mathrm{GeV}$.

Our results for the $\mathcal{N}=0 \mathrm{MHV}$ amplitudes are presented in table III. The full QCD amplitudes can be reconstructed from the $\mathcal{N}=0$ amplitudes and the $\mathcal{N}=4$ and $\mathcal{N}=1$ supersymmetric parts via eq. (2.3). The corresponding supersymmetric amplitudes are listed in tables I and II, using results of refs. [6, 7]. Note that we have extracted an overall factor of $i c_{\Gamma}$ from the numerical values presented in the tables. We do not include the coefficients of the leading $1 / \epsilon^{2}$ singularities of the $\mathcal{N}=4$ amplitudes in the table, as these are easily extracted from the values of tree amplitudes, for any helicity configuration,

$$
\left.A_{n ; 1}^{\mathcal{N}=4}\right|_{1 / \epsilon^{2}}=-\frac{n c_{\Gamma}}{\epsilon^{2}} A_{n}^{\text {tree }}
$$

The numerical values of the tree amplitudes may be read off from the values of the $1 / \epsilon$ singularities of either the $\mathcal{N}=1$ or the $\mathcal{N}=0$ loop amplitudes,

$$
\left.A_{n ; 1}^{\mathcal{N}=1}\right|_{1 / \epsilon}=\frac{c_{\Gamma}}{\epsilon} A_{n}^{\text {tree }},\left.\quad \quad A_{n ; 1}^{\mathcal{N}=0}\right|_{1 / \epsilon}=\frac{c_{\Gamma}}{3 \epsilon} A_{n}^{\text {tree }}
$$

given in tables II and III. Our results in table III at six points match those of ref. [4], taking into account differing phase conventions. The rational terms in our results agree completely with formulae provided by the authors of ref. [52], to high numerical accuracy and for a large number of phase-space points.

\section{ONE-LOOP $n$-GLUON $\mathcal{N}=0$ AMPLITUDES WITH TWO NEGATIVE HELIC- ITIES}

We now turn to the analytic construction of the all- $n$ amplitude. The complete amplitude is decomposed according to,

$$
A_{n ; 1}^{\mathcal{N}=0}(1, m)=c_{\Gamma}\left[\hat{C}_{n}(1, m)+\hat{R}_{n}(1, m)\right],
$$


where 1 and $m$ label the negative-helicity legs. Here $\hat{C}_{n}$ is the appropriate cut completion of $C_{n}$, given in eq. (A1). To calculate $\hat{R}_{n}(1, m)$ we will, as in the examples of the previous section, choose a $[1, m\rangle$ shift. The remaining rational terms are given by eq. (3.23). Using the assumption, extrapolated from the four- and five-gluon cases, that $\operatorname{Inf} A_{n}$ vanishes for a $[1, m\rangle$ shift $^{2}$, we have,

$$
\hat{R}_{n}(1, m)=R_{n}^{D}(1, m)+O_{n}(1, m)-\operatorname{Inf} \hat{C}_{n}(1, m) .
$$

Our first step is to determine the large- $z$ behavior of the cut-completed terms $\hat{C}_{n}(1, m)$. The result of this exercise is given in eq. (A17) of appendix A.

Next we turn our attention to the overlap terms, $O_{n}$. These terms are calculated by taking the residues of the poles in $z$ generated by shifting $\widehat{C R}_{n}$ of eq. (A13) by $[1, m\rangle$. In $O_{n}$ we do not include the residues of poles coming from the tree amplitude which is an overall prefactor of $\widehat{C R}_{n}(1, m)$ in eq. (A13). Such residues would cancel against corresponding terms produced by the insertion of $\widehat{C R}_{n}$ into eq. (5.3) below. Hence we shall drop both types of terms from the following discussion. We refer to these modified overlap terms as $\tilde{O}_{n}$. The final remaining piece necessary for calculating the complete rational term comes from the recursive rational diagrams, $R_{n}^{D}(1, m)$. These terms are computed using an on-shell recursion relation for $\hat{R}_{n}$ generated by our chosen $[1, m\rangle$ shift. The non-zero terms of the recursion relation coming from this shift are,

$$
\begin{aligned}
& \hat{R}_{n}(1, m) \\
& \begin{aligned}
\hat{R}_{n-1}\left(\hat{1}^{-}, \ldots,(m-2)^{+}, \hat{K}_{(m-1) m}^{-},(m\right. & \left.+1)^{+}, \ldots, n^{+}\right) \frac{i}{s_{(m-1) m}} \\
& \times A_{3}^{\text {tree }}\left(-\hat{K}_{(m-1) m}^{+},(m-1)^{+}, \hat{m}^{-}\right)
\end{aligned} \\
& \quad+\hat{R}_{n-1}\left(\hat{1}^{-}, \ldots,(m-1)^{+}, \hat{K}_{m(m+1)}^{-},(m+2)^{+}, \ldots, n^{+}\right) \frac{i}{s_{m(m+1)}} \\
& \quad \times A_{3}^{\text {tree }}\left(-\hat{K}_{m(m+1)}^{+}, \hat{m}^{-},(m+1)^{+}\right) \\
& \quad+A_{s}\left(1^{-}, 2^{+}, \ldots, m^{-}, \ldots, n^{+}\right)+\tilde{O}_{n}(1, m)-\operatorname{Inf} \hat{C}_{n}(1, m) .
\end{aligned}
$$

Here we have combined all the pieces containing known all- $n$ amplitudes $[46,59]$ into $A_{s}$, which is defined by,

$$
A_{s}\left(1^{-}, 2^{+}, \ldots, m^{-}, \ldots, n^{+}\right)
$$

\footnotetext{
${ }^{2}$ As mentioned earlier, the assumption that $\operatorname{Inf} A_{n}$ vanishes in this case can be checked by requiring consistency of the final result with all factorization limits.
} 


$$
\begin{gathered}
=\sum_{j_{1}=2}^{m} \sum_{j_{2}=\max \left(m, j_{1}+2\right)}^{\min \left(n, j_{1}+n-3\right)}\left(A_{n-j_{2}+j_{1}}^{\text {tree }}\left(\hat{1}^{-}, 2^{+}, \ldots,\left(j_{1}-1\right)^{+}, \hat{K}_{j_{1} \ldots j_{2}}^{-},\left(j_{2}+1\right)^{+}, \ldots, n^{+}\right) \frac{i}{s_{j_{1} \ldots j_{2}}}\right. \\
\times R_{j_{2}-j_{1}+2}\left(-\hat{K}_{j_{1} \ldots j_{2}}^{+}, j_{1}^{+}, \ldots, \hat{m}^{-}, \ldots, j_{2}^{+}\right) \\
+R_{n-j_{2}+j_{1}}\left(\hat{1}^{-}, 2^{+}, \ldots,\left(j_{1}-1\right)^{+}, \hat{K}_{j_{1} \ldots j_{2}}^{+},\left(j_{2}+1\right)^{+}, \ldots, n^{+}\right) \frac{i}{s_{j_{1} \ldots j_{2}}} \\
\left.\times A_{j_{2}-j_{1}+2}^{\text {tree }}\left(-\hat{K}_{j_{1} \ldots j_{2}}^{-}, j_{1}^{+}, \ldots, \hat{m}^{-}, \ldots, j_{2}^{+}\right)\right) .
\end{gathered}
$$

As mentioned above, the reason $\hat{R}$ instead of the full rational term $R$ appears on the righthand side of eq. (5.3), is that the additional $\widehat{C R}_{n}$ terms have been cancelled against certain overlap contributions. The three-point loop vertices appearing in eq. (5.4) vanish, $R_{3}\left(\hat{1}^{-}, 2^{+}, \hat{K}_{3 \ldots n}^{+}\right)=R_{3}\left(\hat{1}^{-}, \hat{K}_{2 \ldots(n-1)}^{+}, n^{+}\right)=0$.

The recursive rational contributions naturally split themselves into two classes. The first class consists of the one-loop amplitudes with one negative-helicity leg (which are completely rational) and the tree amplitudes that multiply them. These terms are fully known and are contained in $A_{s}$. The second class of recursive contributions is, however, more difficult. They are given by the first two terms of eq. (5.3) and consist of the rational parts of lower-point one-loop amplitudes with two negative-helicity legs, along with the tree amplitudes that multiply them. Specifically, these unknown amplitudes are exactly those we are trying to determine, having the same number of negative-helicity legs, but with fewer positive-helicity legs. The key for obtaining an all- $n$ expression is to solve recursively for this class of terms.

\section{A. Solving the recursion relation}

To solve eq. (5.3) recursively for $\hat{R}_{n}(1, m)$, we follow the same general plan as given in [44] for the on-shell recursion relations for massive tree-level amplitudes [42, 43]. (Other examples of systematic solutions to on-shell recursion relations may be found in refs. [29, 40, 47].)

Our basic tactic is to insert the left-hand side of eq. (5.3) into the right-hand side of eq. (5.3) repeatedly. At each insertion we find that our desired amplitude $\hat{R}_{n}(1, m)$ appears on the right-hand side with one fewer positive-helicity leg, and multiplied by one more threepoint gluon vertex. This 'unwinding' of the amplitude continues until we have reduced the right-hand side of $\hat{R}_{n}$ (eq. (5.3)) down to, in this case, $\hat{R}_{4}$ and a sum of terms that contain only known quantities ( $A_{s}$, overlap terms $\tilde{O}$ and large- $z$ subtraction terms $\operatorname{Inf} \widehat{C}$ ) multiplied by strings of $A_{3}^{\text {tree }}$ vertices. 
The presence of two terms on the right-hand side of eq. (5.3) containing $\hat{R}_{n-1}$ means that we remove a leg from either side of $m$ at each step of this unwinding. For example, our solution contains the following four terms involving $A_{s}$ at the second unwinding step

$$
\begin{aligned}
& A_{s}\left(\hat{1}^{-}, \ldots,(m-3)^{+}, \hat{K}_{(m-2)\left(\hat{K}_{(m-1) m}\right)}^{-},(m+1)^{+}, \ldots, n^{+}\right) \frac{i}{s_{\left(\hat{K}_{(m-1) m}\right)(m-2)}} \\
& \times A_{3}^{\text {tree }}\left(-\hat{K}_{(m-2)\left(\hat{K}_{(m-1) m}\right)}^{+},(m-2)^{+}, \hat{K}_{(m-1) m}^{-}\right) \frac{i}{s_{(m-1) m}} A_{3}^{\text {tree }}\left(-\hat{K}_{(m-1) m}^{+},(m-1)^{+}, \hat{m}^{-}\right), \\
& A_{s}\left(\hat{1}^{-}, \ldots,(m-2)^{+}, \hat{K}_{\left(\hat{K}_{(m-1) m}\right)(m+1)}^{-},(m+2)^{+}, \ldots, n^{+}\right) \frac{i}{s_{\left(\hat{K}_{(m-1) m}\right)(m+1)}} \\
& \times A_{3}^{\text {tree }}\left(-\hat{K}_{\left(\hat{K}_{(m-1) m}\right)(m+1)}^{+}, \hat{K}_{(m-1) m}^{-},(m+1)^{+}\right) \frac{i}{s_{(m-1) m}} A_{3}^{\text {tree }}\left(-\hat{K}_{(m-1) m}^{+},(m-1)^{+}, \hat{m}^{-}\right) \text {, } \\
& A_{s}\left(\hat{1}^{-}, \ldots,(m-2)^{+}, \hat{K}_{(m-1)\left(\hat{K}_{m(m+1)}^{-}\right)}^{-}(m+2)^{+}, \ldots, n^{+}\right) \frac{i}{s_{(m-1)\left(\hat{K}_{m(m+1)}\right)}} \\
& \times A_{3}^{\text {tree }}\left(-\hat{K}_{(m-1)\left(\hat{K}_{m(m+1)}^{+}\right)}^{+},(m-1)^{+}, \hat{K}_{m(m+1)}^{-}\right) \frac{i}{s_{m(m+1)}} A_{3}^{\text {tree }}\left(-\hat{K}_{m(m+1)}^{+}, \hat{m}^{-},(m+1)^{+}\right), \\
& A_{s}\left(\hat{1}^{-}, \ldots,(m-1)^{+}, \hat{K}_{\left(\hat{K}_{m(m+1)}\right)(m+2)}^{-},(m+3)^{+}, \ldots, n^{+}\right) \frac{i}{s_{\left(\hat{K}_{m(m+1)}\right)(m+2)}} \\
& \times A_{3}^{\text {tree }}\left(-\hat{K}_{\left(\hat{K}_{m(m+1)}\right)(m+2)}^{+}, \hat{K}_{m(m+1)}^{-},(m+2)^{+}\right) \frac{i}{s_{m(m+1)}} A_{3}^{\text {tree }}\left(-\hat{K}_{m(m+1)}^{+}, \hat{m}^{-},(m+1)^{+}\right) .
\end{aligned}
$$

As can be seen, we get a term for each possible order we can extract two legs from around the leg $m$ in the amplitude. Therefore, after extracting $l$ legs (i.e., performing $l$ unwinding steps) we obtain $2^{l}$ terms. Each term corresponds to a particular ordering of the $l$ legs that we extract from around $m$.

At each step of the unwinding we must choose new shifted momenta. We always choose to shift the two negative-helicity legs of $\hat{R}$. For example, after the first step we choose $\left[\hat{1}, \hat{K}_{m(m+1)}\right\rangle$ or $\left[\hat{1}, \hat{K}_{(m-1) m}\right\rangle$ as the shifted legs, depending upon which of the two terms on the right-hand side of eq. (5.3) we insert $\hat{R}_{n-1}$ into. Similarly, when we perform a second insertion, of $\hat{R}_{n-2}$, we choose the intermediate $\hat{K}$ momentum leg of the last shift and the previously shifted $\hat{1}$ leg.

At each step we extract one leg from $\hat{R}_{i}$ and create one extra three-point gluon vertex. The order of extraction can be thought of as a path of extracted legs denoted by the ordered set of momenta $\left\{x_{1}, x_{2}, \ldots, x_{j+1}\right\}$, starting with $x_{1}=k_{m}$. Then $x_{2}$ denotes the momentum of the first extracted leg after $m, x_{3}$ is the momentum of the leg extracted after $x_{2}$, and so on, until we reach the last extracted leg $x_{j+1}$ (if $j$ unwinding steps are performed). We need to define two sequences of momentum sums related to the chain of intermediate momenta appearing 


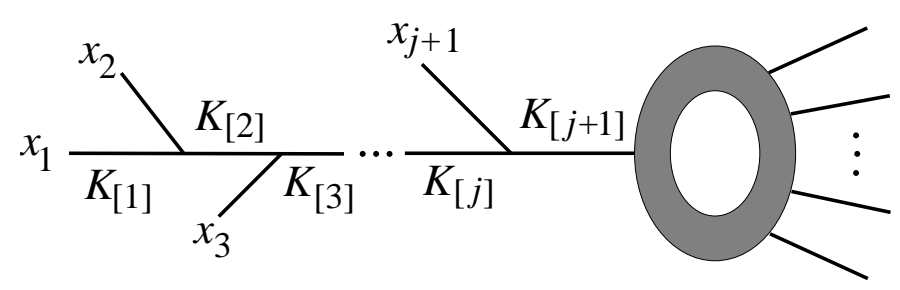

FIG. 10: The propagators corresponding to the sequence of $K_{[i]}$.

as arguments of the three-point gluon vertices. We denote these sequences by $\left\{K_{[i]}\right\}, i=$ $1, \ldots, j+1$ and $\left\{\hat{K}_{[i]}\right\}, i=1, \ldots, j$. We also define a sequence $\left\{\hat{k}_{[i]}\right\}$ of corresponding shifts of $k_{1}$, for $i=0, \ldots, j$. All these momenta are on shell, even $\left\{K_{[i]}\right\}$. The momentum $K_{[i]}$ is defined recursively by adding the external momentum $x_{i}$ to $K_{[i-1]}$ and performing a shift in order to bring the sum back on shell. The shift also alters the propagator leg of the previous unwinding step from $K_{[i-1]}$ to $\hat{K}_{[i-1]}$, keeping it on shell. (See fig. 10.) The sequence of shifted $\left\{\hat{k}_{[i]}\right\}$ is dictated by momentum conservation.

The elements of the sequences involved in the first unwinding step are, for the case $x_{2}=m-1$,

$$
\begin{aligned}
K_{[1]}^{\mu} & =k_{m}^{\mu}, \\
K_{[2]}^{\mu} & =k_{m-1}^{\mu}+k_{m}^{\mu}-\frac{K_{(m-1) m}^{2}\left\langle 1^{-}\left|\gamma^{\mu}\right| k_{m}^{-}\right\rangle}{2\left\langle 1^{-}\left|k_{m-1}\right| k_{m}^{-}\right\rangle} \equiv \hat{K}_{(m-1) m}, \\
\hat{K}_{[1]}^{\mu} & =k_{m}^{\mu}-\frac{K_{(m-1) m}^{2}\left\langle 1^{-}\left|\gamma^{\mu}\right| k_{m}^{-}\right\rangle}{2\left\langle 1^{-}\left|k_{m-1}\right| k_{m}^{-}\right\rangle} \equiv \hat{m}, \\
\hat{k}_{[0]}^{\mu} & =k_{1}^{\mu}, \\
\hat{k}_{[1]}^{\mu} & =k_{1}^{\mu}+\frac{K_{(m-1) m}^{2}\left\langle 1^{-}\left|\gamma^{\mu}\right| k_{m}^{-}\right\rangle}{2\left\langle 1^{-}\left|k_{m-1}\right| k_{m}^{-}\right\rangle} \equiv \hat{1} .
\end{aligned}
$$

In this notation, the expression obtained at the first step of the unwinding process is,

$$
\frac{i A_{3}^{\text {tree }}\left(-K_{[2]}^{+},(m-1)^{+}, \hat{K}_{[1]}^{-}\right)}{K_{(m-1) m}^{2}} A_{s}\left(\hat{k}_{[1]}^{-}, 2^{+}, \ldots,(m-2)^{+}, K_{[2]}^{-},(m+1)^{+}, \ldots, n^{+}\right) .
$$

More generally, after $j$ unwinding steps, we have the following product of $A_{3}^{\text {tree }}$ vertices multiplied by an $A_{s}$ term,

$$
(-1)^{i_{2}-m}\left[\prod_{r=2}^{j+1} \frac{i A_{3}^{\text {tree }}\left(-K_{[r]}^{+}, x_{r}^{+}, \hat{K}_{[r-1]}^{-}\right)}{\left(x_{r}+K_{[r-1]}\right)^{2}}\right] A_{s}\left(\hat{k}_{[j]}^{-}, 2^{+}, \ldots,\left(i_{1}-1\right)^{+}, K_{[j+1]}^{-},\left(i_{2}+1\right)^{+}, \ldots, n^{+}\right),
$$


where the propagators corresponding to the $K_{[i]}$ are depicted in fig. 10. Here $j$ legs have been extracted, $\left(m-i_{1}\right)$ legs from the left of $m$ and $\left(i_{2}-m\right)$ legs from the right of $m$. The factor of $(-1)^{i_{2}-m}$, with $\left(i_{2}-m\right)$ the number of legs $x_{i}$ that are after $m$ in the external ordering, comes from reversing the order of the arguments of the three-point vertices of legs extracted in positions above $m$ in the external ordering, i.e.,

$$
A_{3}^{\text {tree }}\left(-K_{[r]}^{+}, \hat{K}_{[r-1]}^{-}, x_{r}^{+}\right)=(-1) A_{3}^{\text {tree }}\left(-K_{[r]}^{+}, x_{r}^{+}, \hat{K}_{[r-1]}^{-}\right) .
$$

This reordering is needed so that all extracted three-point vertices have the same order as given in eq. (5.8).

The recursive definitions of the sequences $\left\{K_{[i]}\right\},\left\{\hat{K}_{[i]}\right\}$ and $\left\{\hat{k}_{[i]}\right\}$, which satisfy the on-shell requirements, are

$$
\begin{aligned}
K_{[1]}^{\mu} & =k_{m}^{\mu}, \\
K_{[i]}^{\mu} & =x_{i}^{\mu}+K_{[i-1]}^{\mu}+w_{[i]}^{\mu}, \\
\hat{K}_{[i]}^{\mu} & =K_{[i]}^{\mu}+w_{[i+1]}^{\mu}, \\
\hat{k}_{[0]}^{\mu} & =k_{1}^{\mu}, \\
\hat{k}_{[i]}^{\mu} & =\hat{k}_{[i-1]}^{\mu}-w_{[i+1]}^{\mu},
\end{aligned}
$$

where

$$
w_{[i]}^{\mu}=-\frac{\left(x_{i}+K_{[i-1]}\right)^{2}\left\langle\hat{k}_{[i-2]}^{-}\left|\gamma^{\mu}\right| K_{[i-1]}^{-}\right\rangle}{2\left\langle\hat{k}_{[i-2]}^{-}\left|\not_{i}\right| K_{[i-1]}^{-}\right\rangle}=-\frac{\left(x_{i}+K_{[i-1]}\right)^{2}\left\langle 1^{-}\left|\gamma^{\mu}\right| K_{[i-1]}^{-}\right\rangle}{2\left\langle 1^{-}\left|\not_{i}\right| K_{[i-1]}^{-}\right\rangle} .
$$

We can make the identification on the right-hand side of eq. (5.11) because the choice of shifts is such that the unshifted spinors at step $i$ are given by,

$$
\left\langle\hat{k}_{[i]}^{-}\right|=\left\langle\hat{k}_{[i-1]}^{-}\right|=\cdots=\left\langle 1^{-}\right|, \quad\left\langle\hat{K}_{[i]}^{+}\right|=\left\langle\hat{K}_{[i-1]}^{+}\right|=\cdots=\left\langle m^{+}\right| .
$$

The shifted spinors at step $i$ are given by

$$
\left\langle\hat{k}_{[i]}^{+}\right|=\left\langle\hat{k}_{[i-1]}^{+}\right|+\frac{\left(x_{i+1}+K_{[i]}\right)^{2}}{\left\langle 1^{-}\left|\not_{i+1}\right| K_{[i]}^{-}\right\rangle}\left\langle K_{[i]}^{+}\right|, \quad\left\langle\hat{K}_{[i]}^{-}\right|=\left\langle K_{[i]}^{-}\right|-\frac{\left(x_{i+1}+K_{[i]}\right)^{2}}{\left\langle 1^{-}\left|\not_{i+1}\right| K_{[i]}^{-}\right\rangle}\left\langle K_{[i]}^{-}\right| .
$$

From eq. (5.10) we can see that momentum conservation is satisfied,

$$
\hat{k}_{[i-1]}^{\mu}+K_{[i]}^{\mu}=\hat{k}_{[i-2]}^{\mu}+x_{i}^{\mu}+K_{[i-1]}^{\mu}=\cdots=k_{1}^{\mu}+\sum_{\ell=1}^{i} x_{\ell}^{\mu} .
$$


Using the representation of the one-loop $n$-gluon amplitudes with one negative helicity in ref. [46], one can see that the left-handed spinor $\tilde{\lambda}_{1}$ for the negative-helicity leg (leg 1) never appears. From eq. (5.12), the right-handed spinor $\lambda_{1}$ that does appear is unshifted, $\lambda_{\hat{k}_{[i]}}=\left\langle\hat{k}_{[i]}^{-}\right|=\left\langle 1^{-}\right|$.

Next, using the identity,

$$
\prod_{r=2}^{j+1} \frac{i A_{3}^{\text {tree }}\left(-K_{[r]}^{+}, x_{r}^{+}, \hat{K}_{[r-1]}^{-}\right)}{\left(x_{r}+K_{[r-1]}\right)^{2}}=i \frac{A_{j+2}^{\text {tree }}\left(1^{-}, x_{j+1}^{+}, x_{j}^{+}, \ldots, x_{2}^{+}, m^{-}\right)}{\left\langle 1 K_{[j+1]}\right\rangle^{2}}
$$

we rewrite the general term $(5.8)$ as

$$
\begin{aligned}
& (-1)^{\left(i_{2}-m\right)} A_{j+2}^{\text {tree }}\left(1^{-}, x_{j+1}^{+}, x_{j}^{+}, \ldots, x_{2}^{+}, m^{-}\right) \frac{i}{\left\langle 1 K_{[j+1]}\right\rangle^{2}} \\
& \quad \times A_{s}\left(1^{-}, 2^{+}, \ldots,\left(i_{1}-1\right)^{+}, K_{[j+1]}^{-},\left(i_{2}+1\right)^{+}, \ldots, n^{+}\right) .
\end{aligned}
$$

If $A_{s}$ contains a $\lambda_{m}$ spinor (where $m$ is the negative-helicity leg of $A_{s}$, other than leg 1 ) then we rewrite the general form of this term as

$$
\begin{aligned}
& (-1)^{\left(i_{2}-m\right)} A_{j+2}^{\text {tree }}\left(1^{-}, x_{j+1}^{+}, x_{j}^{+}, \ldots, x_{2}^{+}, m^{-}\right) \frac{i}{\left\langle 1 K_{[j+1]}\right\rangle^{2}} \\
& \quad \times A_{s}^{\prime}\left(1^{-}, 2^{+}, \ldots,\left(i_{1}-1\right)^{+}, K_{[j+1]}^{-},\left(i_{2}+1\right)^{+}, \ldots, n^{+}\right) f(j+1) .
\end{aligned}
$$

Here $A_{s}^{\prime}$ is defined to contain no $\lambda_{m}$ spinors; we construct $f(j+1)$ such that it contains all such factors. The argument $(j+1)$ denotes the dependence of this function on the momentum of the last leg that was extracted for this term in the unwinding, $x_{j+1}$. This step is necessitated by our desire to combine together, into a single simple form, all terms containing the same set of extracted legs. The terms involved have to be independent of the order of extraction of these legs, for it to be possible to combine them.

Looking at eqs. (5.16) and (5.17) we see immediately that $K_{[j]}$ depends upon the order of the path of extracted legs. Because of the homogeneity of the spinors in the amplitude, the denominator factor of $\left\langle 1 K_{[j]}\right\rangle^{2}$ must combine with a complementary factor term in the quantity $A_{s}$ multiplying it, to generate spinor strings of the form $\left\langle 1^{-}\right| K_{[j]} \ldots$. This string can be rewritten using $\left\langle 1^{-}\right| K_{\left(\hat{K}_{a \ldots b}\right) c}=\left\langle 1^{-}\right| K_{a \ldots b c}$ to get $\left\langle 1^{-}\right| K_{i_{1} \ldots i_{2}} \ldots=\left\langle 1^{-}\right| K_{i_{1} \ldots i_{2}} \ldots$ (because the spinor $\lambda_{1}$ in the shift always contracts with $\left\langle 1^{-}\right|$to give $\left.\langle 11\rangle=0\right)$. Now, $K_{i_{1} \ldots i_{2}}$ is just the consecutive sum of momenta between the left-most extracted leg $i_{1}$ and the right-most extracted leg $i_{2}$. So these terms become independent of the order of the extracted legs in the unwinding, and henceforth we will simply replace any such appearance of $K_{[j]}$ with $\hat{K}_{i_{1} \ldots i_{2}}$. 
However, this is not the only source of a path dependence. The presence of a $\lambda_{m}$ spinor in $A_{s}$ also leads to such a dependence, for example a $\langle y m\rangle$ term in $A_{s}$ becomes $\left\langle y K_{[j+1]}\right\rangle$ during the unwinding. We find that we cannot completely eliminate the path dependence coming from a $\langle y m\rangle$ term. We can only reduce it to that of the last extracted leg. To see this, consider again $\left\langle y K_{[j+1]}\right\rangle$ as it will appear in the amplitude combined with $\left\langle 1 K_{[j+1]}\right\rangle$,

$$
\frac{\left\langle y K_{[j+1]}\right\rangle\left[K_{[j+1]} K_{[j]}\right]}{\left\langle 1 K_{[j+1]}\right\rangle\left[K_{[j+1]} K_{[j]}\right]} \equiv \frac{\left\langle y x_{j+1}\right\rangle\left[x_{j+1} K_{[j]}\right]}{\left\langle 1 x_{j+1}\right\rangle\left[x_{j+1} K_{[j]}\right]}=\frac{\left\langle y x_{j+1}\right\rangle}{\left\langle 1 x_{j+1}\right\rangle} .
$$

The factor of $\left\langle 1 K_{[j+1]}\right\rangle$ in the denominator here is always guaranteed to be present due to the homogeneity of the spinors. From this example we see that, after removing any dependence upon the shifted external momentum from the amplitude, we can distinguish different paths of extracted legs only by the last leg extracted from $\hat{R}$. For the amplitudes we consider here we find that such $\lambda_{m}$ spinors are always present, so to proceed we must first isolate all $\lambda_{m}$ as in eq. (5.17). Once they are isolated, we can straightforwardly reduce the path-dependence to that of the final extracted leg only.

With this simplified dependence upon the extracted path we can now proceed to combine together all terms containing the same set of extracted legs $\left\{i_{1}, \ldots, m, \ldots, i_{2}\right\}$. All terms that correspond to each possible path between two legs are combined. For example, considering the case when $i_{1}=m-1$ and $i_{2}=m+1$, we combine the middle two terms of eq. (5.5), as these correspond to the two possible ways of extracting the set of legs $\{m-1, m, m+1\}$. In general for the set of extracted legs $\left\{i_{1}, \ldots, i_{2}\right\}$ we see that the last leg extracted from such a contributing term can only be either $i_{1}$ or $i_{2}$. Therefore we write the sum of all possible extraction paths of the legs $\left\{i_{1}, \ldots, m, \ldots, i_{2}\right\}$ as two sums, one for $x_{j+1}=k_{i_{1}}$ and one for $x_{j+1}=k_{i_{2}}$, to get,

$$
\begin{aligned}
& \left(f\left(i_{1}\right)(-1)^{n_{\beta_{1}}} \sum_{\sigma \in O P\left(\alpha_{1}, \beta_{1}\right)} A_{i_{2}-i_{1}+2}^{\text {tree }}\left(1^{-}, i_{1}^{+}, \sigma\left(\alpha_{1}, \beta_{1}\right), m^{-}\right)\right. \\
- & \left.f\left(i_{2}\right)(-1)^{\left(n_{\beta_{1}}-1\right)} \sum_{\sigma \in O P\left(\alpha_{2}, \beta_{2}\right)} A_{i_{2}-i_{1}+2}^{\text {tree }}\left(1^{-}, i_{2}^{+}, \sigma\left(\alpha_{2}, \beta_{2}\right), m^{-}\right)\right) \\
& \times \frac{i}{\left\langle 1 \hat{K}_{i_{1} \ldots i_{2}}\right\rangle^{2}} A_{s}^{\prime}\left(1^{-}, 2^{+}, \ldots,\left(i_{1}-1\right)^{+}, \hat{K}_{i_{1} \ldots i_{2}}^{-},\left(i_{2}+1\right)^{+}, \ldots, n^{+}\right) \\
= & \left(f\left(i_{1}\right) \frac{\langle 1 m\rangle^{3}}{\left\langle 1 i_{1}\right\rangle\left\langle i_{1} m\right\rangle^{3}}(-1)^{n_{\beta_{1}}} \sum_{\sigma \in O P\left(\alpha_{1}, \beta_{1}\right)} A_{i_{2}-i_{1}+1}^{\text {tree }}\left(i_{1}^{-}, \sigma\left(\alpha_{1}, \beta_{1}\right), m^{-}\right)\right. \\
& \left.-f\left(i_{2}\right) \frac{\langle 1 m\rangle^{3}}{\left\langle 1 i_{2}\right\rangle\left\langle i_{2} m\right\rangle^{3}}(-1)^{n_{\beta_{2}}} \sum_{\sigma \in O P\left(\alpha_{2}, \beta_{2}\right)} A_{i_{2}-i_{1}+1}^{\text {tree }}\left(i_{2}^{-}, \sigma\left(\alpha_{2}, \beta_{2}\right), m^{-}\right)\right)
\end{aligned}
$$




$$
\times \frac{i}{\left\langle 1 \hat{K}_{i_{1} \ldots i_{2}}\right\rangle^{2}} A_{s}^{\prime}\left(1^{-}, 2^{+}, \ldots,\left(i_{1}-1\right)^{+}, \hat{K}_{i_{1} \ldots i_{2}}^{-},\left(i_{2}+1\right)^{+}, \ldots, n^{+}\right),
$$

where $\sigma\left(\alpha_{i}, \beta_{i}\right)$ indicates one of the possible 'ordered permutations (OP)' or 'mergings' of the ordered sets $\alpha_{i}$ and $\beta_{i}$, with $\alpha_{1}=\left\{i_{1}+1, \ldots, m-1\right\}, \beta_{1}=\left\{i_{2}, \ldots, m+1\right\}, \alpha_{2}=\left\{i_{1}, \ldots, m-1\right\}$ and $\beta_{2}=\left\{i_{2}-1, \ldots, m+1\right\}$. The elements of each merging are the union of the two sets, $\alpha_{i} \cup \beta_{i} \equiv\left\{x_{j}, \ldots, x_{2}\right\}$. The merged ordering must preserve the order of the elements $\alpha_{i}$ and $\beta_{i}$ individually, but any relative ordering of elements of $\alpha_{i}$ with respect to those of $\beta_{i}$ is permitted. Also, $n_{\beta_{i}}$ is the number of elements in the set $\beta_{i}$. We can then rewrite eq. (5.19) using the Kleiss-Kuijf relation [60] between tree amplitudes (for which a simple proof was given in ref. [61]). The Kleiss-Kuijf relation reads, in our notation,

$$
A_{n}^{\text {tree }}\left(1, \alpha, n, \beta^{T}\right)=(-1)^{n_{\beta}} \sum_{\sigma \in \mathrm{OP}(\alpha, \beta)} A_{n}^{\text {tree }}(1, \sigma(\alpha, \beta), n),
$$

where $\beta^{T}$ is $\beta$ written in the reversed order. Applying it to eq. (5.19) and rearranging the resulting MHV tree amplitudes to restore gluon 1, we obtain,

$$
\begin{aligned}
& \frac{\left\langle i_{2} 1\right\rangle\left\langle i_{1} m\right\rangle f\left(i_{1}\right)-\left\langle i_{1} 1\right\rangle\left\langle i_{2} m\right\rangle f\left(i_{2}\right)}{\langle 1 m\rangle\left\langle i_{2} i_{1}\right\rangle} A_{i_{2}-i_{1}+2}^{\text {tree }}\left(1^{-}, i_{1}^{+}, \ldots, m^{-}, \ldots, i_{2}^{+}\right) \\
& \times \frac{i}{\left\langle 1 \hat{K}_{i_{1} \ldots i_{2}}\right\rangle^{2}} A_{s}^{\prime}\left(1^{-}, 2^{+}, \ldots,\left(i_{1}-1\right)^{+}, \hat{K}_{i_{1} \ldots i_{2}}^{-},\left(i_{2}+1\right)^{+}, \ldots, n^{+}\right) .
\end{aligned}
$$

The final result is then given by summing eq. (5.21) over all possible sets of extracted legs $\left\{i_{1}, \ldots, m, \ldots, i_{2}\right\}$, which is equivalent to summing over all possible factorization channels of the amplitude.

In the simplest case, $A_{s}$ contains no $\lambda_{m}$ spinors and $f(i)=1$. In this case, using the Schouten identity, eq. (5.21) reduces to

$$
\begin{aligned}
& A_{i_{2}-i_{1}+2}^{\text {tree }}\left(1^{-}, i_{1}^{+}, \ldots, m^{-}, \ldots, i_{2}^{+}\right) \\
& \quad \times \frac{i}{\left\langle 1 \hat{K}_{i_{1} \ldots i_{2}}\right\rangle^{2}} A_{s}\left(1^{-}, 2^{+}, \ldots,\left(i_{1}-1\right)^{+}, \hat{K}_{i_{1} \ldots i_{2}}^{-},\left(i_{2}+1\right)^{+}, \ldots, n^{+}\right) .
\end{aligned}
$$

The case of the amplitude considered here is more complicated. We always have to take into account a $\lambda_{m}$ spinor contained in the denominator factor of $s_{j_{1} \ldots \hat{m}_{\ldots} \ldots j_{2}}$ in the $A_{s}$ of eq. (5.4). During the unwinding, $s_{j_{1} \ldots \hat{m} \ldots j_{2}}$ becomes $s_{j_{1} \ldots K_{[j+1]} \ldots j_{2}}$, which, using eq. (5.18), can be rewritten as

$$
\begin{aligned}
s_{j_{1} \ldots K_{[j+1]} \ldots j_{2}} & =\left\langle K_{[j+1]}^{-}\left|K_{j_{1} \ldots\left(i_{1}-1\right)\left(i_{2}+1\right) \ldots j_{2}}\right| K_{[j+1]}^{-}\right\rangle+s_{j_{1} \ldots\left(i_{1}-1\right)\left(i_{2}+1\right) \ldots j_{2}} \\
& =\frac{\left\langle x_{j+1}^{-}\left|\mathcal{G}_{j_{1}, i_{1}, i_{2}, j_{2}}^{\dagger}\right| 1^{+}\right\rangle}{\left\langle x_{j+1} 1\right\rangle}
\end{aligned}
$$


where we define,

$$
\begin{aligned}
\mathcal{G}_{a, b, c, d} & =K_{a \ldots d} K_{a \ldots(b-1)(c+1) \ldots d} \\
\mathcal{G}_{a, b, c, d}^{\dagger} & =K_{a \ldots(b-1)(c+1) \ldots d} K_{a \ldots d} .
\end{aligned}
$$

and again $x_{j+1}$ is the last leg extracted during the unwinding.

Hence, if we isolate a factor of $1 / s_{j_{1} \ldots \hat{m}_{\ldots j}}$ from the remainder of $A_{s}$ (along the lines of eq. (5.17)), then we see that $f(j+1)$ for the final extracted leg $x_{j+1}$ is given by

$$
f(j+1)=\frac{\left\langle x_{j+1} 1\right\rangle}{\left\langle x_{j+1}^{-}\left|\mathcal{G}_{j_{1}, i_{1}, i_{2}, j_{2}}^{\dagger}\right| 1^{+}\right\rangle} .
$$

Inserting this result for $f(j+1)$ into eq. (5.21) and taking into account the cases when $i_{1}=m$ or $i_{2}=m$ we get the result for a specific set of extracted legs $\left\{i_{1}, \ldots, m, \ldots, i_{2}\right\}$,

$$
\begin{gathered}
-\sum_{j_{1}=2}^{i_{1}} \sum_{j_{2}=\max \left(i_{2}, i_{2}+2-i_{1}+j_{1}\right)}^{\min \left(n, n-3+j_{1}\right)} \mathcal{K}\left(j_{1}, j_{2}, i_{1}, i_{2}, m\right) A_{i_{2}-i_{1}+2}^{\text {tree }}\left(1^{-}, i_{1}^{+}, \ldots, m^{-}, \ldots, i_{2}^{+}\right) \frac{1}{\left\langle 1 \hat{K}_{i_{1} \ldots i_{2}}\right\rangle^{2}} \\
\times A_{s}^{\prime} j_{1 j_{2}}\left(1^{-}, 2^{+}, \ldots,\left(i_{1}-1\right)^{+}, \hat{K}_{i_{1} \ldots i_{2}}^{-},\left(i_{2}+1\right)^{+}, \ldots, n^{+}\right),
\end{gathered}
$$

with

$$
\mathcal{K}\left(a, b, i_{1}, i_{2}, m\right)= \begin{cases}i_{1}=m \text { and } i_{2}=m: & 1 / s_{a \ldots b}, \\ i_{1}=m: & \left\langle i_{2} 1\right\rangle /\left\langle i_{2}{ }^{-}\left|\mathcal{G}_{a, i_{1}, i_{2}, b}^{\dagger}\right| 1^{+}\right\rangle \\ i_{2}=m: & \left\langle 1 i_{1}\right\rangle /\left\langle 1^{-}\left|\mathcal{G}_{a, i_{1}, i_{2}, b}\right| i_{1}{ }^{+}\right\rangle \\ \text {otherwise }: & \frac{\left\langle m^{-}\left|\mathcal{G}_{a, i_{1}, i_{2}, b}^{\dagger}\right| 1^{+}\right\rangle\left\langle 1 i_{1}\right\rangle\left\langle i_{2} 1\right\rangle}{\langle m 1\rangle\left\langle 1^{-}\left|\mathcal{G}_{a, i_{1}, i_{2}, b}\right| i_{1}{ }^{+}\right\rangle\left\langle i_{2}{ }^{-}\left|\mathcal{G}_{a, i_{1}, i_{2}, b}^{\dagger}\right| 1^{+}\right\rangle}\end{cases}
$$

and

$$
\begin{aligned}
& A_{s}^{\prime}{ }^{j_{1} j_{2}}\left(1^{-}, 2^{+}, \ldots, m^{-}, \ldots, n^{+}\right) \\
& \equiv A_{n-j_{2}+j_{1}}^{\text {tree }}\left(\hat{1}^{-}, 2^{+}, \ldots,\left(j_{1}-1\right)^{+}, \hat{K}_{j_{1} \ldots j_{2}}^{-},\left(j_{2}+1\right)^{+}, \ldots, n^{+}\right) \\
& \times R_{j_{2}-j_{1}+2}\left(-\hat{K}_{j_{1} \ldots j_{2}}^{+}, j_{1}^{+}, \ldots, \hat{m}^{-}, \ldots, j_{2}^{+}\right) \\
& +R_{n-j_{2}+j_{1}}\left(\hat{1}^{-}, 2^{+}, \ldots,\left(j_{1}-1\right)^{+}, \hat{K}_{j_{1} \ldots j_{2}}^{+},\left(j_{2}+1\right)^{+}, \ldots, n^{+}\right) \\
& \times A_{j_{2}-j_{1}+2}^{\text {tree }}\left(-\hat{K}_{j_{1} \ldots j_{2}}^{-}, j_{1}^{+}, \ldots, \hat{m}^{-}, \ldots, j_{2}^{+}\right),
\end{aligned}
$$

which are the terms of $A_{s}$ with a factor of $i$ and the denominator factors of $s_{j_{1} \ldots\left(\hat{K}_{i_{1} \ldots i_{2}}\right) \ldots j_{2}}$ removed. 
To obtain the final result we must include all possible factorization channels. We also must include the overlap and $\operatorname{Inf} \hat{C}_{n}$ terms. Finally, there is a contribution from the terminal step of the unwinding, because $\hat{R}_{4}$ is non-zero for this amplitude. The complete result for $\hat{R}_{n}$ is then given by,

$$
\begin{aligned}
& \hat{R}_{n}(1, m) \quad \sum_{i_{1}=2}^{m} \sum_{i_{2}=m}^{m \min \left(n, n+i_{1}-5\right)} A_{i_{2}-i_{1}+2}^{\text {tree }}\left(1^{-}, i_{1}^{+}, \ldots, m^{-}, \ldots, i_{2}^{+}\right) \frac{1}{\left\langle 1 \hat{K}_{i_{1} \ldots i_{2}}\right\rangle^{2}}[ \\
& +i \operatorname{Inf} \hat{C}_{n-i_{2}+i_{1}}\left(1^{-}, 2^{+}, \ldots,\left(i_{1}-1\right)^{+}, \hat{K}_{i_{1} \ldots i_{2}}^{-},\left(i_{2}+1\right)^{+}, \ldots, n^{+}\right) \\
& \quad+\sum_{j_{1}=2}^{i_{1}} \sum_{j_{2}=\max \left(i_{2}, i_{2}+2-i_{1}+j_{1}\right)}^{\min \left(n, n-3+j_{1}\right)} \mathcal{K}\left(j_{1}, j_{2}, i_{1}, i_{2}, m\right) \\
& \quad \times\left(A_{s}^{\prime} j_{1 j_{2}}\left(1^{-}, 2^{+}, \ldots,\left(i_{1}-1\right)^{+}, \hat{K}_{i_{1} \ldots i_{2}}^{-},\left(i_{2}+1\right)^{+}, \ldots, n^{+}\right)\right. \\
& \left.\left.\quad+\tilde{O}_{n-i_{2}+i_{1}}^{\prime j_{1} j_{2}}\left(1^{-}, 2^{+}, \ldots,\left(i_{1}-1\right)^{+}, \hat{K}_{i_{1} \ldots i_{2}}^{-},\left(i_{2}+1\right)^{+}, \ldots, n^{+}\right)\right)\right] \\
& -A_{n-2}^{\text {tree }}\left(1^{-}, 3^{+}, \ldots,(m-1)^{+}, m^{-},(m+1)^{+}, \ldots,(n-1)^{+}\right) \frac{1}{\langle n 2\rangle^{2}} \\
& +\frac{2}{9} A_{n}^{\text {tree }}(1, m) .
\end{aligned}
$$

Here $A_{s}^{\prime} j_{1} j_{2}$ is as given in eq. (5.28) and in a corresponding treatment $\tilde{O}^{\prime} j_{1} j_{2}$ represents the terms of $\tilde{O}$ containing denominator factors of $s_{j_{1} \ldots\left(\hat{K}_{i_{1} \ldots i_{2}}\right) \ldots j_{2}}$, but with those factors extracted; as in $A_{s}^{\prime} j_{1} j_{2}$ we also extract an overall factor of $i$. Furthermore, as mentioned above, $\tilde{O}^{\prime} j_{1} j_{2}$ does not include terms that stem from the residues of poles of the overall tree term in $\widehat{C R}_{n}$, because they are precisely cancelled by $\widehat{C R}_{n}$. For this reason, $\widehat{C R}_{n}$ is not included in eq. (5.29). Finally, note that for $i_{1}=i_{2}=m$, the 'tree amplitude' in eq. (5.29) is to be evaluated as $A_{2}^{\text {tree }}\left(1^{-}, m^{-}\right)=i\langle 1 m\rangle^{4} /(\langle 1 m\rangle\langle m 1\rangle)=-i\langle 1 m\rangle^{2}$.

\section{B. The final result}

After combining all the necessary terms into eq. (5.29) we find that the final result for $\hat{R}_{n}$ is given by,

$$
\begin{aligned}
& \hat{R}_{n}(1, m) \\
& =A_{n}^{\text {tree }}(1, m) \\
& \quad \times\left\{\sum _ { i _ { 1 } = 2 } ^ { m } \sum _ { i _ { 2 } = m } ^ { \operatorname { m i n } ( n , n + i _ { 1 } - 5 ) } \left(\sum_{j_{1}=2}^{i_{1}} \sum_{j_{2}=\max \left(i_{2}, i_{2}+2-i_{1}+j_{1}\right)}^{\min \left(n, n-3+j_{1}\right)} \frac{\mathcal{K}\left(j_{1}, j_{2}, i_{1}, i_{2}, m\right)}{3} \frac{\left\langle\left(i_{1}-1\right) i_{1}\right\rangle\left\langle i_{2}\left(i_{2}+1\right)\right\rangle}{\left\langle 1 i_{1}\right\rangle\left\langle i_{2} 1\right\rangle}\right.\right.
\end{aligned}
$$




$$
\begin{aligned}
& \times \frac{h_{1 ; j_{1} j_{2}}^{i_{1} i_{2}}(n) h_{2 ; j_{1} j_{2}}^{i_{1} i_{2}}(n)\left(T_{1 ; j_{1} j_{2}}^{i_{1} i_{2}}(n)+T_{2 ; j_{1} j_{2}}^{i_{1} i_{2}}(n)+s_{j_{1} \ldots\left(i_{1}-1\right),\left(i_{2}+1\right) \ldots j_{2}}^{4}\left(T_{3 ; j_{1} j_{2}}^{i_{1} i_{2}}(n)+T_{4 ; j_{1} j_{2}}^{i_{1} i_{2}}(n)\right)\right)}{\left\langle\left(j_{1}-1\right)^{-}\left|\overline{\mathcal{G}}_{j_{1}, i_{1}, i_{2}, j_{2}}\right| 1^{-}\right\rangle\left\langle 1^{-}\left|\overline{\mathcal{G}}_{j_{1}, i_{1}, i_{2}, j_{2}}^{\dagger}\right|\left(j_{2}+1\right)^{-}\right\rangle} \\
& \left.\left.+T_{6}^{i_{1} i_{2}}(m, n)+T_{7}^{i_{1} i_{2}}(n)\right)+T_{5}(m, n)\right\}
\end{aligned}
$$

where we have introduced

$$
\begin{aligned}
& \overline{\mathcal{G}}_{a, b, c, d}=\mathbb{K}_{a \ldots(b-1),(c+1) \ldots d} \mathbb{K}_{b \ldots c}, \\
& \overline{\mathcal{G}}_{a, b, c, d}^{\dagger}=\mathbb{K}_{b \ldots c} K_{a \ldots(b-1),(c+1) \ldots d},
\end{aligned}
$$

in addition to the definitions in eq. (5.24). This solution then depends on the functions,

$$
\begin{aligned}
& h_{1 ; j_{1} j_{2}}^{i_{1} i_{2}}(n)=\left\{\begin{array}{l}
j_{1}=i_{1}: 1 / s_{\left(i_{2}+1\right) \ldots j_{2}}, \\
j_{1}<i_{1}:-\frac{\left\langle\left(j_{1}-1\right) j_{1}\right\rangle\left\langle 1^{-}|\overline{\mathcal{G}}| 1^{+}\right\rangle}{\left\langle 1^{-}\left|\overline{\mathcal{G}}^{\dagger}\right| j_{1}{ }^{+}\right\rangle\left\langle\left(i_{1}-1\right)^{-}\left|\mathcal{G}^{\dagger}\right| 1^{+}\right\rangle},
\end{array}\right. \\
& h_{2 ; j_{1} j_{2}}^{i_{1} i_{2}}(n)=\left\{\begin{array}{l}
j_{2}=i_{2}: 1 / s_{j_{1} \ldots i_{1}-1}, \\
j_{2}>i_{2}: \frac{\left\langle j_{2}\left(j_{2}+1\right)\right\rangle\left\langle 1^{-}|\overline{\mathcal{G}}| 1^{+}\right\rangle}{\left\langle j_{2}^{-}|\overline{\mathcal{G}}| 1^{+}\right\rangle\left\langle 1^{-}|\mathcal{G}|\left(i_{2}+1\right)^{+}\right\rangle},
\end{array}\right.
\end{aligned}
$$

where above and throughout this section $\mathcal{G} \equiv \mathcal{G}_{j_{1}, i_{1}, i_{2}, j_{2}}, \mathcal{G}^{\dagger} \equiv \mathcal{G}_{j_{1}, i_{1}, i_{2}, j_{2}}, \overline{\mathcal{G}} \equiv \overline{\mathcal{G}}_{j_{1}, i_{1}, i_{2}, j_{2}}$ and $\overline{\mathcal{G}}^{\dagger} \equiv \overline{\mathcal{G}}_{j_{1}, i_{1}, i_{2}, j_{2}}^{\dagger}$. The function $T_{1}$ is given by,

$$
T_{1 ; j_{1} j_{2}}^{i_{1} i_{2}}(n)=\sum_{l=i_{2}+1}^{j_{2}} f_{1 ; j_{1} j_{2}}^{i_{1} i_{2}}(l, n)+\sum_{l=j_{1}-1}^{i_{1}-2} f_{1 ; j_{1} j_{2}}^{i_{1} i_{2}}(l, n),
$$


with

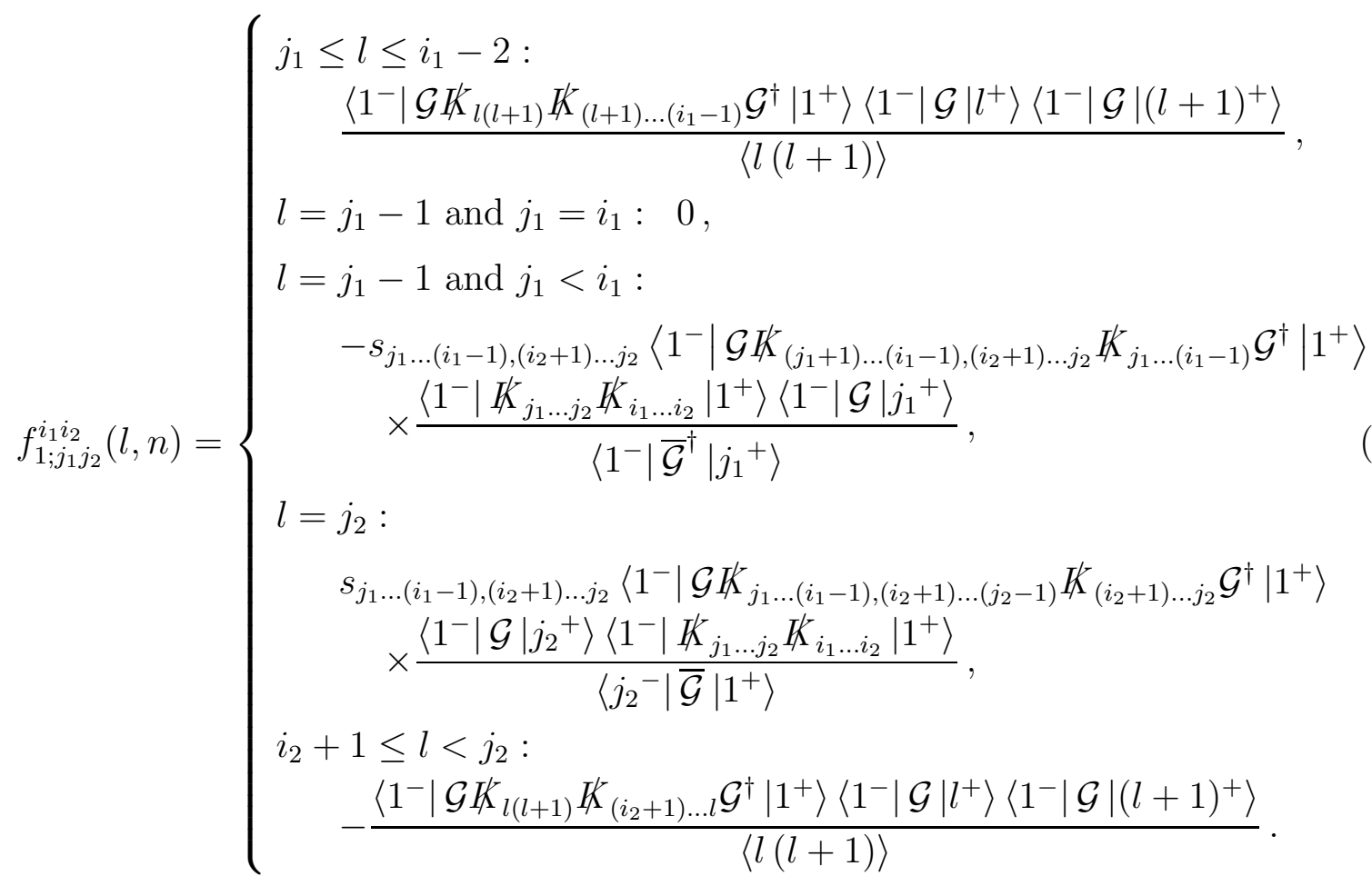

Next, the $T_{2}$ term reads,

$$
T_{2 ; j_{1} j_{2}}^{i_{1} i_{2}}(n)=\sum_{l=i_{2}+2}^{j_{2}} f_{2 ; j_{1} j_{2}}^{i_{1} i_{2}}(l, n)+\sum_{l=j_{1}-1}^{i_{1}-3} f_{2 ; j_{1} j_{2}}^{i_{1} i_{2}}(l, n)
$$


with

$$
\begin{aligned}
& f_{2 ; j_{1} j_{2}}^{i_{1} i_{2}}(l, n)= \\
& j_{1}<l \leq\left(i_{1}-1\right): \\
& -\sum_{p=l+1}^{i_{1}-2} \frac{\langle(l-1) l\rangle\left\langle 1^{-}\left|\mathcal{G} K_{l \ldots p} K_{(p+1) \ldots\left(i_{1}-1\right)} \mathcal{G}^{\dagger}\right| 1^{+}\right\rangle^{3}}{\left\langle 1^{-}\left|\mathcal{G} K_{(p+1) \ldots\left(i_{1}-1\right)} K_{l \ldots p}\right|(l-1)^{+}\right\rangle\left\langle 1^{-}\left|\mathcal{G} K_{(p+1) \ldots\left(i_{1}-1\right)} K_{l \ldots p}\right| l^{+}\right\rangle} \\
& \times \frac{\langle p(p+1)\rangle\left\langle 1^{-}\left|\mathcal{G} K_{l \ldots\left(i_{1}-1\right)}[\mathcal{F}(l, p)]^{2} K_{(p+1) \ldots\left(i_{1}-1\right)} \mathcal{G}^{\dagger}\right| 1^{+}\right\rangle}{s_{l \ldots p}\left\langle 1^{-}\left|\mathcal{G} K_{l \ldots\left(i_{1}-1\right)} K_{l \ldots p}\right| p^{+}\right\rangle\left\langle 1^{-}\left|\mathcal{G} K_{l \ldots\left(i_{1}-1\right)} K_{l \ldots p}\right|(p+1)^{+}\right\rangle}, \\
& l=j_{1}: \\
& -\sum_{p=j_{1}+1}^{i_{1}-2} \frac{\left\langle 1^{-}\left|\overline{\mathcal{G}}^{\dagger}\right| j_{1}{ }^{+}\right\rangle\left\langle 1^{-}\left|\mathcal{G} K_{j_{1} \ldots p} K_{(p+1) \ldots\left(i_{1}-1\right)} \mathcal{G}^{\dagger}\right| 1^{+}\right\rangle^{3}}{\left\langle 1^{-}\left|\mathcal{G} K_{(p+1) \ldots\left(i_{1}-1\right)} K_{j_{1} \ldots p} \overline{\mathcal{G}}\right| 1^{+}\right\rangle\left\langle 1^{-}\left|\mathcal{G} K_{(p+1) \ldots\left(i_{1}-1\right)} K_{j_{1} \ldots p}\right| j_{1}{ }^{+}\right\rangle} \\
& \times \frac{\langle p(p+1)\rangle\left\langle 1^{-}\left|\mathcal{G} K_{j_{1} \ldots\left(i_{1}-1\right)}\left[\mathcal{F}\left(j_{1}, p\right)\right]^{2} K_{(p+1) \ldots\left(i_{1}-1\right)} \mathcal{G}^{\dagger}\right| 1^{+}\right\rangle}{s_{j_{1} \ldots p}\left\langle 1^{-}\left|\mathcal{G} K_{j_{1 \ldots\left(i_{1}-1\right)}} K_{j_{1 \ldots p}}\right| p^{+}\right\rangle\left\langle 1^{-}\left|\mathcal{G} K_{j_{1} \ldots\left(i_{1}-1\right)} K_{j_{1} \ldots p}\right|(p+1)^{+}\right\rangle}, \\
& l=j_{1}-1 \text { and }\left(j_{1} \geq i_{1}-1 \text { or } j_{2}=i_{2}\right): 0 \text {, } \\
& l=j_{1}-1 \text { and }\left(j_{1}<i_{1}-1 \text { and } j_{2}>i_{2}\right) \text { : } \\
& -\sum_{p=j_{1}}^{i_{1}-2} \frac{\left\langle 1^{-}\left|K_{j_{1} \ldots j_{2}} K_{i_{1} \ldots i_{2}}\right| 1^{+}\right\rangle\left\langle j_{2}^{-}\left|K_{j_{1} \ldots\left(i_{1}-1\right),\left(i_{2}+1\right) \ldots j_{2}} K_{i_{1} \ldots i_{2}}\right| 1^{+}\right\rangle}{\left\langle 1^{-}\left|\mathcal{G}_{j_{1}, p+1, j_{2}, j_{2}} \overline{\mathcal{G}}_{p+1, i_{1}, i_{2}, j_{2}}\right| 1^{+}\right\rangle} \\
& \left\langle 1^{-}\left|\mathcal{G} K_{(p+1) \ldots\left(i_{1}-1\right),\left(i_{2}+1\right) \ldots j_{2}} K_{(p+1) \ldots\left(i_{1}-1\right)} \mathcal{G}^{\dagger}\right| 1^{+}\right\rangle^{3} \\
& \times \overline{\left\langle 1^{-}\left|\mathcal{G} K_{(p+1) \ldots\left(i_{1}-1\right)} U_{j_{1} j_{2}}^{i_{1} i_{2}}\left(p, j_{2}+1\right)\right| j_{2}{ }^{+}\right\rangle\left\langle 1^{-}\left|\mathcal{G} K_{(p+1) \ldots\left(i_{1}-1\right)} K_{j_{1} \ldots p} \overline{\mathcal{G}}\right| 1^{+}\right\rangle} \\
& \times \frac{\langle p(p+1)\rangle\left\langle 1^{-}\left|\mathcal{G} K_{\left(i_{2}+1\right) \ldots j_{2}}\left[F_{-; j_{1} j_{2}}^{i_{1} i_{2}}\left(j_{1}, p\right)\right]^{2} K_{(p+1) \ldots\left(i_{1}-1\right)} \mathcal{G}^{\dagger}\right| 1^{+}\right\rangle}{\left\langle 1^{-}\left|\mathcal{G} K_{\left(i_{2}+1\right) \ldots j_{2}} U_{j_{1} j_{2}}^{i_{1} i_{2}}\left(p, j_{2}+1\right)\right| p^{+}\right\rangle\left\langle 1^{-}\left|\mathcal{G} K_{\left(i_{2}+1\right) \ldots j_{2}} U_{j_{1} j_{2}}^{i_{1} i_{2}}\left(p, j_{2}+1\right)\right|(p+1)^{+}\right\rangle},
\end{aligned}
$$

$i_{2}+1 \leq l \leq j_{2}$ :

$$
\sum_{p=l+1}^{j_{2}} \bar{f}_{2 ; j_{1} j_{2}}^{i_{1} i_{2}}(l, p, n)+\sum_{p=j_{1}-1}^{i_{1}-2} \bar{f}_{2 ; j_{1} j_{2}}^{i_{1} i_{2}}(l, p, n),
$$


and

$$
\begin{aligned}
& \bar{f}_{2 ; j_{1} j_{2}}^{i_{1} i_{2}}(l, p, n)= \\
& \left(j_{1}-1<p \leq\left(i_{1}-1\right):\right. \\
& \frac{\left\langle 1^{-}\left|K_{j_{1} \ldots j_{2}} K_{i_{1} \ldots i_{2}}\right| 1^{+}\right\rangle}{\left\langle 1^{-}\left|\mathcal{G}_{j_{1}, p+1, l-1, j_{2}} \overline{\mathcal{G}}_{p+1, i_{1}, i_{2}, l-1}\right| 1^{+}\right\rangle} \\
& \times \frac{\langle(l-1) l\rangle\left\langle 1^{-}\left|\mathcal{G} K_{(p+1) \ldots\left(i_{1}-1\right),\left(i_{2}+1\right) \ldots(l-1)} \mathbb{K}_{(p+1) \ldots\left(i_{1}-1\right)} \mathcal{G}^{\dagger}\right| 1^{+}\right\rangle^{3}}{\left\langle 1^{-}\left|\mathcal{G} K_{(p+1) \ldots\left(i_{1}-1\right)} U_{j_{1} j_{2}}^{i_{1} i_{2}}(p, l)\right|(l-1)^{+}\right\rangle\left\langle 1^{-}\left|\mathcal{G} K_{(p+1) \ldots\left(i_{1}-1\right)} U_{j_{1} j_{2}}^{i_{1} i_{2}}(p, l)\right| l^{+}\right\rangle} \\
& \times \frac{\langle p(p+1)\rangle\left\langle 1^{-}\left|\mathcal{G} K_{\left(i_{2}+1\right) \ldots(l-1)}\left[F_{-; j_{1} j_{2}}^{i_{1} i_{2}}\left(l, j_{2} ; j_{1}, p\right)\right]^{2} K_{(p+1) \ldots\left(i_{1}-1\right)} \mathcal{G}^{\dagger}\right| 1^{+}\right\rangle}{\left\langle 1^{-}\left|\mathcal{G} K_{\left(i_{2}+1\right) \ldots(l-1)} U_{j_{1} j_{2}}^{i_{1} i_{2}}(p, l)\right| p^{+}\right\rangle\left\langle 1^{-}\left|\mathcal{G} K_{\left(i_{2}+1\right) \ldots(l-1)} U_{j_{1} j_{2}}^{i_{1} i_{2}}(p, l)\right|(p+1)^{+}\right\rangle}, \\
& p=j_{1}-1 \text { and } j_{1}=i_{1}: 0 \text {, } \\
& p=j_{1}-1 \text { and } j_{1}<i_{1} \text { : } \\
& \left\langle 1^{-}\left|K_{j_{1} \ldots j_{2}} K_{i_{1} \ldots i_{2}}\right| 1^{+}\right\rangle \\
& \overline{\left\langle 1^{-}\left|\mathcal{G}_{j_{1}, j_{1}, l-1, j_{2}} \overline{\mathcal{G}}_{j_{1}, i_{1}, i_{2}, l-1}\right| 1^{+}\right\rangle\left\langle 1^{-}\left|\mathcal{G} K_{j_{1} \ldots\left(i_{1}-1\right)} U_{j_{1} j_{2}}^{i_{1} i_{2}}\left(j_{1}-1, l\right)\right|(l-1)^{+}\right\rangle} \\
& \times \frac{\langle(l-1) l\rangle\left\langle 1^{-}\left|\mathcal{G} K_{j_{1} \ldots\left(i_{1}-1\right),\left(i_{2}+1\right) \ldots(l-1)} K_{j_{1} \ldots\left(i_{1}-1\right)} \mathcal{G}^{\dagger}\right| 1^{+}\right\rangle^{3}}{\left\langle 1^{-}\left|\mathcal{G} K_{j_{1} \ldots\left(i_{1}-1\right)} U_{j_{1} j_{2}}^{i_{1} i_{2}}\left(j_{1}-1, l\right)\right| l^{+}\right\rangle} \\
& \times \frac{\left\langle 1^{-}\left|\overline{\mathcal{G}}^{\dagger}\right| j_{1}{ }^{+}\right\rangle\left\langle 1^{-}\left|\mathcal{G} K_{\left(i_{2}+1\right) \ldots(l-1)}\left[\overline{\mathcal{F}}_{-; j_{1} j_{2}}^{i_{1} i_{2}}\left(l, j_{2}\right)\right]^{2} K_{j_{1} \ldots\left(i_{1}-1\right)} \mathcal{G}^{\dagger}\right| 1^{+}\right\rangle}{\left\langle 1^{-}\left|\mathcal{G} K_{\left(i_{2}+1\right) \ldots(l-1)} K_{l \ldots j_{2}} \overline{\mathcal{G}}\right| 1^{+}\right\rangle\left\langle 1^{-}\left|\mathcal{G} K_{\left(i_{2}+1\right) \ldots(l-1)} U_{j_{1} j_{2}}^{i_{1} i_{2}}\left(j_{1}-1, l\right)\right| j_{1}{ }^{+}\right\rangle}, \\
& (l+1) \leq p<j_{2}: \\
& \frac{\langle(l-1) l\rangle\left\langle 1^{-}\left|\mathcal{G} K_{l \ldots p} K_{\left(i_{2}+1\right) \ldots p} \mathcal{G}^{\dagger}\right| 1^{+}\right\rangle^{3}}{\left\langle 1^{-}\left|\mathcal{G} K_{\left(i_{2}+1\right) \ldots p} K_{l \ldots p}\right|(l-1)^{+}\right\rangle\left\langle 1^{-}\left|\mathcal{G} K_{\left(i_{2}+1\right) \ldots p} K_{l \ldots p}\right| l^{+}\right\rangle} \\
& \times \frac{\langle p(p+1)\rangle\left\langle 1^{-}\left|\mathcal{G} K_{\left(i_{2}+1\right) \ldots(l-1)}[\mathcal{F}(l, p)]^{2} K_{\left(i_{2}+1\right) \ldots p} \mathcal{G}^{\dagger}\right| 1^{+}\right\rangle}{s_{l \ldots p}\left\langle 1^{-}\left|\mathcal{G} K_{\left(i_{2}+1\right) \ldots(l-1)} K_{l \ldots p}\right| p^{+}\right\rangle\left\langle 1^{-}\left|\mathcal{G} K_{\left(i_{2}+1\right) \ldots(l-1)} K_{l \ldots p}\right|(p+1)^{+}\right\rangle}, \\
& p=j_{2} \text { : } \\
& \frac{\langle(l-1) l\rangle\left\langle 1^{-}\left|\mathcal{G} K_{l \ldots j_{2}} K_{\left(i_{2}+1\right) \ldots j_{2}} \mathcal{G}^{\dagger}\right| 1^{+}\right\rangle^{3}}{\left\langle 1^{-}\left|\mathcal{G} K_{\left(i_{2}+1\right) \ldots j_{2}} K_{l \ldots j_{2}}\right|(l-1)^{+}\right\rangle\left\langle 1^{-}\left|\mathcal{G} K_{\left(i_{2}+1\right) \ldots j_{2}} K_{l \ldots j_{2}}\right| l^{+}\right\rangle} \\
& \times \frac{\left\langle j_{2}{ }^{-}|\overline{\mathcal{G}}| 1^{+}\right\rangle\left\langle 1^{-}\left|\mathcal{G} K_{\left(i_{2}+1\right) \ldots(l-1)}\left[\mathcal{F}\left(l, j_{2}\right)\right]^{2} K_{\left(i_{2}+1\right) \ldots j_{2}} \mathcal{G}^{\dagger}\right| 1^{+}\right\rangle}{s_{l \ldots j_{2}}\left\langle 1^{-}\left|\mathcal{G} K_{\left(i_{2}+1\right) \ldots(l-1)} K_{l \ldots j_{2}}\right| j_{2}{ }^{+}\right\rangle\left\langle 1^{-}\left|\mathcal{G} K_{\left(i_{2}+1\right) \ldots(l-1)} K_{l \ldots j_{2}} \overline{\mathcal{G}}\right| 1^{+}\right\rangle} .
\end{aligned}
$$

The $T_{3}$ term is given by,

$$
T_{3 ; j_{1} j_{2}}^{i_{1} i_{2}}(n)=\sum_{l=2}^{j_{1}} f_{3 ; j_{1} j_{2}}^{i_{1} i_{2}}(l, n)+\sum_{l=j_{2}+1}^{n-1} f_{3 ; j_{1} j_{2}}^{i_{1} i_{2}}(l, n),
$$


which depends on

$$
f_{3 ; j_{1} j_{2}}^{i_{1} i_{2}}(l, n)=\left\{\begin{array}{l}
2 \leq l<j_{1}-1:-\frac{\left\langle 1^{-}\left|K_{l \ldots(l+1)} K_{1 \ldots l}\right| 1^{+}\right\rangle\langle 1 l\rangle\langle 1(l+1)\rangle}{\langle l(l+1)\rangle} \\
l=j_{1}-1:-\frac{\left\langle 1^{-}\left|K_{\left(j_{1}-1\right) \ldots j_{2}} K_{1 \ldots\left(j_{1}-1\right)}\right| 1^{+}\right\rangle\left\langle 1\left(j_{1}-1\right)\right\rangle\left\langle 1^{-}|\overline{\mathcal{G}}| 1^{+}\right\rangle}{\left\langle\left(j_{1}-1\right)^{-}|\overline{\mathcal{G}}| 1^{+}\right\rangle} \\
l=j_{1} \text { and } j_{2}=n: 0, \\
l=j_{1} \text { and } j_{2}<n: \\
\quad \frac{\left\langle 1^{-}\left|K_{j_{1} \ldots\left(j_{2}+1\right)} K_{\left(j_{2}+1\right) \ldots n}\right| 1^{+}\right\rangle\left\langle 1^{-}|\overline{\mathcal{G}}| 1^{+}\right\rangle\left\langle 1\left(j_{2}+1\right)\right\rangle}{\left\langle 1^{-}\left|\overline{\mathcal{G}}^{\dagger}\right|\left(j_{2}+1\right)^{+}\right\rangle} \\
j_{2}+1 \leq l \leq n-1: \frac{\left\langle 1^{-}\left|K_{l \ldots(l+1)} K_{(l+1) \ldots n}\right| 1^{+}\right\rangle\langle 1 l\rangle\langle 1(l+1)\rangle}{\langle l(l+1)\rangle}
\end{array}\right.
$$

Following on, the $T_{4}$ term reads,

$$
T_{4 ; j_{1} j_{2}}^{i_{1} i_{2}}(n)=\sum_{l=3}^{j_{1}} f_{4 ; j_{1} j_{2}}^{i_{1} i_{2}}(l, n)+\sum_{l=j_{2}+1}^{n-2} f_{4 ; j_{1} j_{2}}^{i_{1} i_{2}}(l, n)
$$


with

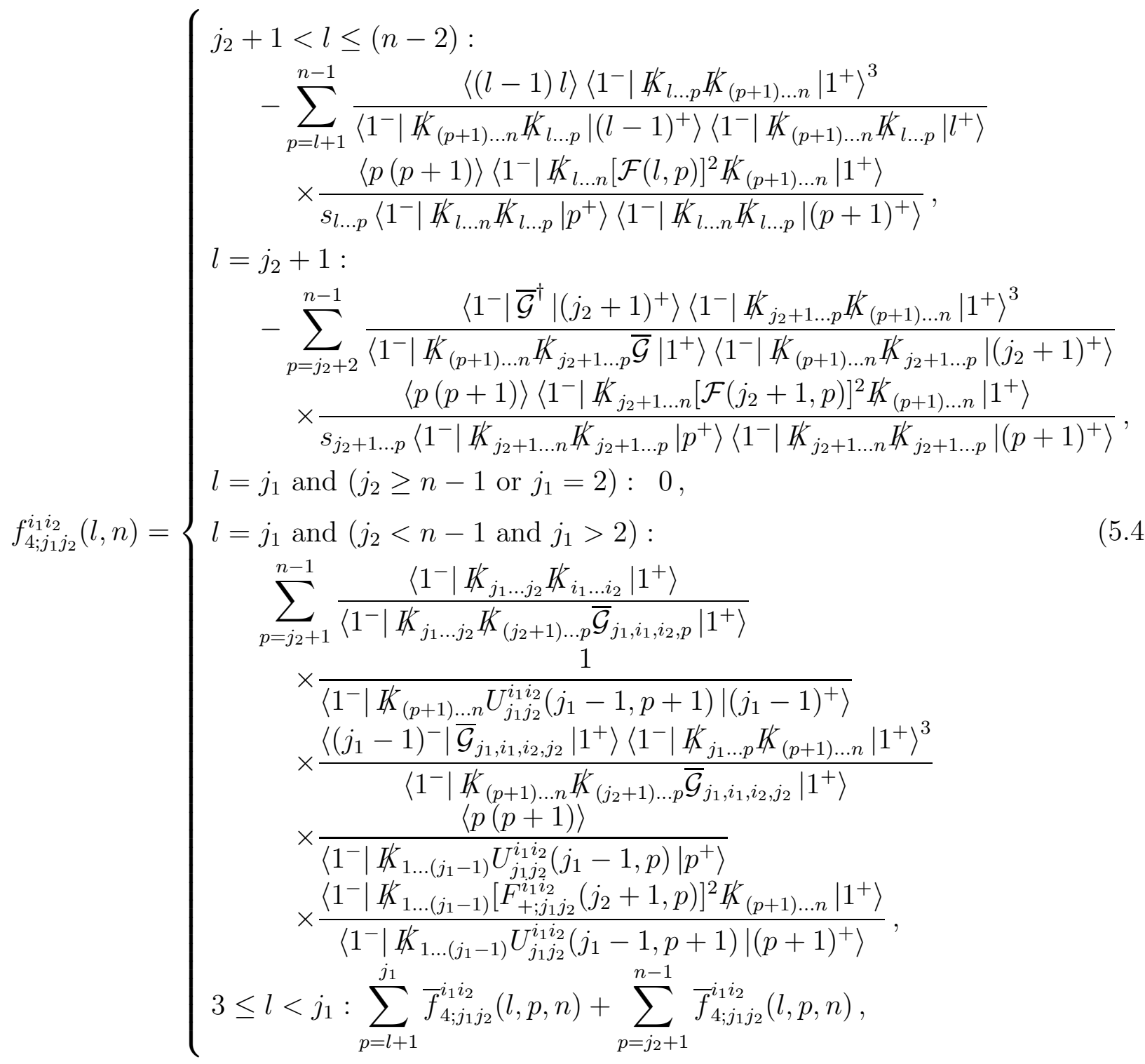


and

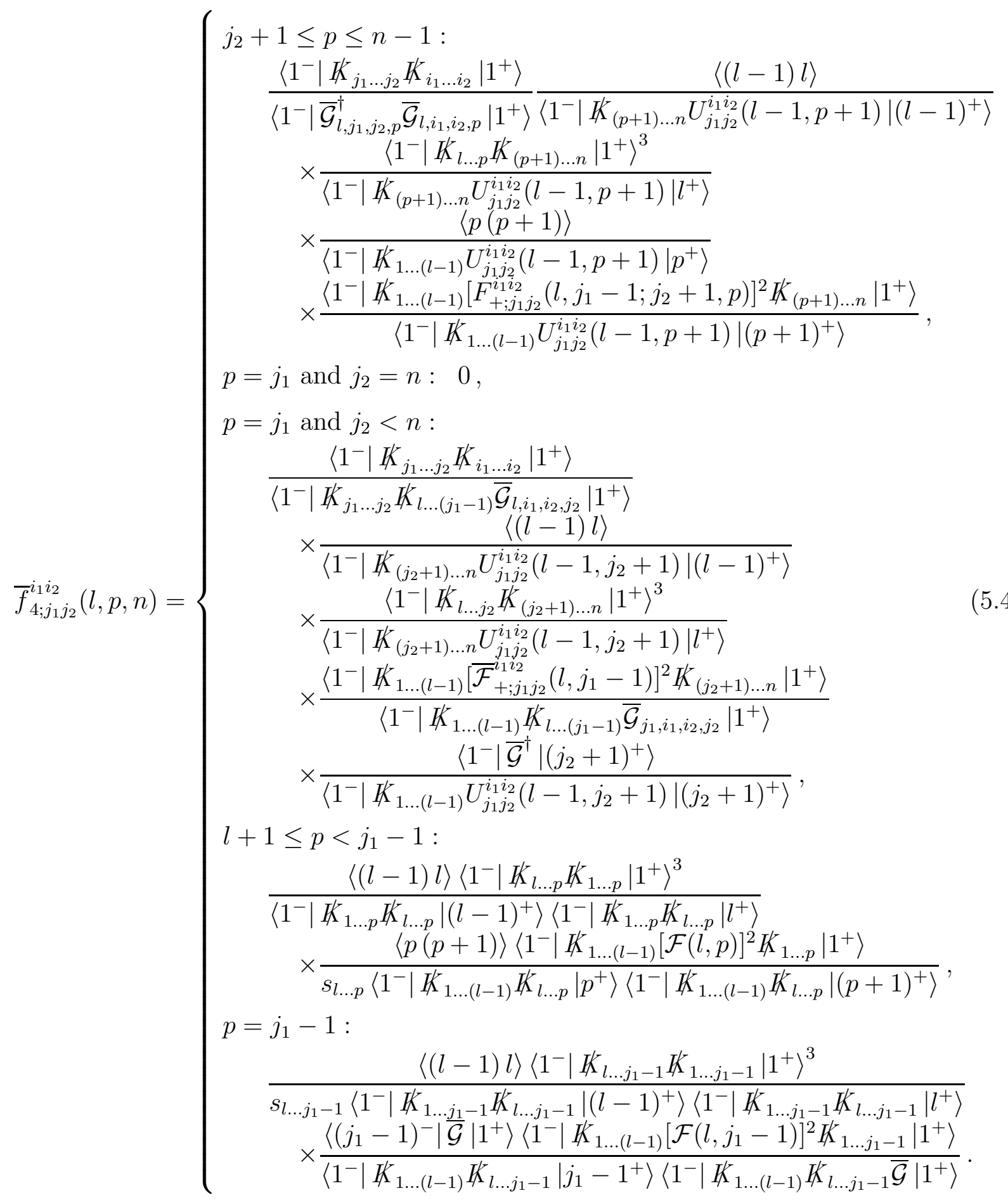

Above we have used the following abbreviations,

$$
U_{j_{1} j_{2}}^{i_{1} i_{2}}(p, l)=K_{(p+1) \ldots\left(i_{1}-1\right),\left(i_{2}+1\right) \ldots(l-1)}-\frac{K_{i_{1} \ldots i_{2}}\left|1^{+}\right\rangle\left\langle 1^{-}\right| \mathcal{G}}{\left\langle 1^{-}\left|K_{j_{1} \ldots j_{2}} K_{i_{1} \ldots i_{2}}\right| 1^{+}\right\rangle},
$$

as well as

$$
F_{ \pm ; j_{1} j_{2}}^{i_{1} i_{2}}(a, b)=\mathcal{F}(a, b) \mp \frac{K_{j_{1} \ldots j_{2}}\left|1^{+}\right\rangle\left\langle 1^{-}\right| \overline{\mathcal{G}}^{\dagger}}{\left\langle 1^{-}\left|K_{j_{1} \ldots j_{2}} K_{i_{1} \ldots i_{2}}\right| 1^{+}\right\rangle} K_{a \ldots b}
$$




$$
\begin{aligned}
\overline{\mathcal{F}}_{ \pm ; j_{1} j_{2}}^{i_{1} i_{2}}(a, b) & =\mathcal{F}(a, b) \pm K_{a \ldots b} \frac{\overline{\mathcal{G}}\left|1^{+}\right\rangle\left\langle 1^{-}\right| K_{j_{1} \ldots j_{2}}}{\left\langle 1^{-}\left|K_{j_{1} \ldots j_{2}} K_{i_{1} \ldots i_{2}}\right| 1^{+}\right\rangle}, \\
F_{ \pm ; j_{1} j_{2}}^{i_{1} i_{2}}(a, b ; c, d) & =\overline{\mathcal{F}}_{ \pm ; j_{1} j_{2}}^{i_{1} i_{2}}(a, b)+F_{ \pm ; j_{1} j_{2}}^{i_{1} i_{2}}(c, d)+K_{a \ldots b} K_{c \ldots d} \\
\mathcal{F}(a, b) & =\sum_{i=a}^{b-1} \sum_{m=i+1}^{b} \not k_{i} k_{m} .
\end{aligned}
$$

Next we have,

$$
T_{5}(m, n)= \begin{cases}m=2 \text { or } m=n: & \frac{2}{9}, \\ \text { otherwise }: & \frac{2}{9}-\frac{\langle 12\rangle\langle 23\rangle\langle(n-1) n\rangle\langle n 1\rangle}{\langle 2 n\rangle^{2}\langle 13\rangle\langle(n-1) 1\rangle} .\end{cases}
$$

The unwinding of eq. (A13) gives the following contribution,

$$
\begin{aligned}
& T_{6}^{i_{1} i_{2}}(m, n)=\frac{1}{2} \sum_{j_{1}=1}^{i_{1}-1} \sum_{j_{2}=\max \left(i_{2}, i_{2}+2-i_{1}+j_{1}\right)}^{\min \left(n, n+j_{1}-2\right)} \sum_{r=2}^{3} \frac{\mathcal{K}\left(j_{1}+1, j_{2}, i_{1}, i_{2}, m\right)\left\langle\left(i_{1}-1\right) i_{1}\right\rangle\left\langle i_{2}\left(i_{2}+1\right)\right\rangle}{\left\langle 1 i_{1}\right\rangle\left\langle i_{2} 1\right\rangle} \\
& \times\left(\mathcal{H}_{+; j_{1} j_{2}}^{i_{1} i_{2}}\left(r, j_{1}, j_{2}+1\right)+\mathcal{H}_{-; j_{1} j_{2}}^{i_{1} i_{2}}\left(r, j_{1}+1, j_{2}+1\right)-\mathcal{H}_{+; j_{1} j_{2}}^{i_{1} i_{2}}\left(r, j_{1}+1, j_{2}\right)-\mathcal{H}_{-; j_{1} j_{2}}^{i_{1} i_{2}}\left(r, j_{1}, j_{2}\right)\right) .
\end{aligned}
$$

The terms entering this expression are given by,

$$
\mathcal{H}_{ \pm ; j_{1} j_{2}}^{i_{1} i_{2}}(r, a, b)=\left(H_{j_{1} j_{2}}^{i_{1} i_{2}}(r, a, b) \pm \bar{H}_{j_{1} j_{2}}^{i_{1} i_{2}}(r, b, a)\right)
$$

where $\mathcal{H}_{i_{1}-1 j_{2}}^{i_{1} i_{2}}\left(r, j_{1}+1, y\right)=\mathcal{H}_{i_{1}-1 j_{2}}^{i_{1} i_{2}}\left(r, i_{2}, y\right)$. We have introduced the following functions,

$$
\begin{aligned}
\bar{H}_{j_{1} j_{2}}^{i_{1} i_{2}}(3, a, b) & =-\frac{1}{3} \frac{\mathcal{C}_{j_{1} j_{2}}^{i_{1} i_{2}}(a, b, a)}{\left\langle a^{-}\left|\overline{\mathcal{G}}_{j_{1}+1, i_{1}, i_{2}, j_{2}}\right| 1^{+}\right\rangle^{2}}, \quad \bar{H}_{j_{1} j_{2}}^{i_{1} i_{2}}(2, a, b)=-\frac{1}{2} \frac{\mathcal{S}_{j_{1} j_{2}}^{i_{1} i_{2}}(a, b, a)}{\left\langle a^{-}\left|\overline{\mathcal{G}}_{j_{1}+1, i_{1}, i_{2}, j_{2}}\right| 1^{+}\right\rangle}, \\
H_{j_{1} j_{2}}^{i_{1} i_{2}}(3, a, b) & =\frac{1}{3} \frac{\mathcal{C}_{j_{1} j_{2}}^{i_{1} i_{2}}(a, a, b)}{\left\langle a^{-}\left|\overline{\mathcal{G}}_{j_{1}+1, i_{1}, i_{2}, j_{2}}\right| 1^{+}\right\rangle^{2}}, \quad H_{j_{1} j_{2}}^{i_{1} i_{2}}(2, a, b)=-\frac{1}{2} \frac{\mathcal{S}_{j_{1} j_{2}}^{i_{1} i_{2}}(a, a, b)}{\left\langle a^{-}\left|\overline{\mathcal{G}}_{j_{1}+1, i_{1}, i_{2}, j_{2}}\right| 1^{+}\right\rangle},(5 .
\end{aligned}
$$

with the constraints $H_{j_{1} j_{2}}^{i_{1} i_{2}}\left(r, i_{2}, x\right)=0, \bar{H}_{j_{1} j_{2}}^{i_{1} i_{2}}\left(r, i_{2}, x\right)=0, \bar{H}_{j_{1} j_{2}}^{i_{1} i_{2}}\left(r, x, i_{2}\right)=0$ and $H_{j_{1} j_{2}}^{i_{1} i_{2}}\left(2, x, i_{2}\right)=0$ as well as $H_{j_{1} j_{2}}^{i_{1} i_{2}}(r, x, y)=0=\bar{H}_{j_{1} j_{2}}^{i_{1} i_{2}}(r, x, y)$ when $K_{j_{1}+1 \ldots i_{1}-1, i_{2}+1 \ldots j_{2}}=k_{x}$. We also need

$$
\begin{aligned}
\mathcal{C}_{j_{1} j_{2}}^{i_{1} i_{2}}(j, a, b)= & -f_{\mathcal{C}_{;} j_{1} j_{2}}^{i_{1} i_{2}}(b, a, b) f_{\mathcal{D}_{j} j_{1} j_{2}}^{i_{1} i_{2}}(j)\left\langle 1^{-}\left|\mathcal{G}_{j_{1}+1, i_{1}, i_{2}, j_{2}}\right| j^{+}\right\rangle \\
& \times\left(\left\langle 1^{-}\left|\mathcal{G}_{j_{1}+1, i_{1}, i_{2}, j_{2}}\right| j^{+}\right\rangle+s_{\left(j_{1}+1\right) \ldots\left(i_{1}-1\right),\left(i_{2}+1\right) \ldots j_{2}}\langle 1 j\rangle\right) \\
\mathcal{S}_{j_{1} j_{2}}^{i_{1} i_{2}}(j, a, b)= & 2 f_{\mathcal{D} ; j_{1} j_{2}}^{i_{1} i_{2}}(j) \frac{\langle 1 a\rangle\langle 1 b\rangle\left\langle 1^{-}\left|\mathcal{G}_{j_{1}+1, i_{1}, i_{2}, j_{2}}\right| a^{+}\right\rangle\left\langle 1^{-}\left|\mathcal{G}_{j_{1}+1, i_{1}, i_{2}, j_{2}}\right| b^{+}\right\rangle}{\langle a b\rangle^{2}\left\langle 1^{-}\left|K_{\left(j_{1}+1\right) \ldots j_{2}} K_{i_{1} \ldots i_{2}}\right| 1^{+}\right\rangle} \\
& \times\left\langle 1^{-}\left|\mathcal{G}_{j_{1}+1, i_{1}, i_{2}, j_{2}}\right| j^{+}\right\rangle,
\end{aligned}
$$


and

$$
\begin{aligned}
& f_{\mathcal{C} ; j_{1} j_{2}}^{i_{1} i_{2}}(j, a, b)= \begin{cases}j=i_{2}: & -\left\langle 1^{-}\left|\overline{\mathcal{G}}_{j_{1}+1, i_{1}, i_{2}, j_{2}}\right| 1^{+}\right\rangle, \\
\text {otherwise }: & \langle 1 b\rangle\left\langle 1^{-}\left|\mathcal{G}_{j_{1}+1, i_{1}, i_{2}, j_{2}}\right| a^{+}\right\rangle /\langle a b\rangle,\end{cases} \\
& f_{\mathcal{D} ; j_{1} j_{2}}^{i_{1} i_{2}}(j)= \begin{cases}j_{1}=i_{1}-1 \text { and } j_{2}=i_{2}+1: & \frac{\left\langle 1^{-}\left|\not k_{j}\right|\left(i_{2}+1\right)^{-}\right\rangle}{\left\langle\left(i_{1}-1\right)^{-}\left|\mathcal{G}_{j_{1}+1, i_{1}, i_{2}, j_{2}}^{\dagger}\right| 1^{+}\right\rangle}, \\
j_{1}=i_{1}-2 \text { and } j_{2}=i_{2}: & -\frac{\left\langle 1^{-}\left|\not k_{j}\right|\left(i_{1}-1\right)^{-}\right\rangle}{\left\langle 1^{-}\left|\mathcal{G}_{j_{1}+1, i_{1}, i_{2}, j_{2}}\right|\left(i_{2}+1\right)^{+}\right\rangle}, \\
\text {otherwise : } & \frac{s_{\left(j_{1}+1\right) \ldots\left(i_{1}-1\right),\left(i_{2}+1\right) \ldots j_{2}}\left\langle 1^{-}\left|K_{j_{1}+1 \ldots j_{2}}\right| k_{j} \mid 1^{+}\right\rangle}{\left.\left\langle\left(i_{1}-1\right)^{-}\left|\mathcal{G}_{j_{1}+1, i_{1}, i_{2}, j_{2}}^{\dagger}\right| 1^{+}\right\rangle\left\langle 1^{-}\left|\mathcal{G}_{j_{1}+1, i_{1}, i_{2}, j_{2}}\right| i_{2}+1\right)^{+}\right\rangle} .\end{cases}
\end{aligned}
$$

Finally, the large- $z$ contribution eq. (A17) results in,

$$
\begin{aligned}
& T_{7}^{i_{1} i_{2}}(n)= \\
& \quad \sum_{j_{1}=2}^{i_{1}-1} \sum_{j_{2}=i_{2}+1}^{n} \frac{\left\langle\left(i_{1}-1\right) i_{1}\right\rangle\left\langle i_{2}\left(i_{2}+1\right)\right\rangle}{\left\langle 1\left(i_{2}+1\right)\right\rangle\left\langle 1 i_{1}\right\rangle\left\langle i_{2} 1\right\rangle\left\langle\left(i_{1}-1\right) 1\right\rangle}\left(\frac{\left\langle 1 j_{1}\right\rangle^{3}\left\langle 1 j_{2}\right\rangle^{3}\left[j_{2} j_{1}\right]^{2}}{\left\langle j_{1} j_{2}\right\rangle^{2}\left\langle j_{1}{ }^{+}\left|K_{i_{1} \ldots i_{2}}\right| 1^{+}\right\rangle\left\langle j_{2}{ }^{+}\left|K_{i_{1} \ldots i_{2}}\right| 1^{+}\right\rangle}\right. \\
& \left.\quad-R_{+; j_{1} j_{2}}^{i_{1} i_{2}}\left(j_{1}, j_{2}\right)+R_{+; j_{1} j_{2}}^{i_{1} i_{2}}\left(j_{1}+1, j_{2}-1\right)+R_{-; j_{1} j_{2}}^{i_{1} i_{2}}\left(j_{1}, j_{2}-1\right)-R_{-; j_{1} j_{2}}^{i_{1} i_{2}}\left(j_{1}+1, j_{2}\right)\right),
\end{aligned}
$$

where we set $R_{ \pm ; j_{1} j_{2}}^{i_{1} i_{2}}(a, b)=0$ if $K_{a \ldots b}^{2}=0$ or if $K_{a \ldots b}=K_{i_{1} \ldots i_{2}}$; otherwise $R_{ \pm ; j_{1} j_{2}}^{i_{1} i_{2}}(a, b)$ is given by

$$
\begin{array}{r}
R_{ \pm ; j_{1}, j_{2}}^{i_{1} i_{2}}(a, b)=\frac{\left\langle 1 j_{1}\right\rangle^{2}\left\langle 1 j_{2}\right\rangle^{2}}{2\left\langle j_{1} j_{2}\right\rangle^{2}\left\langle 1^{-}\left|K_{a \ldots b} K_{i_{1} \ldots i_{2}}\right| 1^{+}\right\rangle}\left(\frac{\left\langle 1^{-}\left|\not K_{a \ldots b} \not k_{j_{1}}\right| 1^{+}\right\rangle^{2}}{\left\langle 1^{-}\left|\not k_{j_{1}} K_{i_{1} \ldots i_{2}}\right| 1^{+}\right\rangle} \pm \frac{\left\langle 1^{-}\left|\not K_{a \ldots b} \not k_{j_{2}}\right| 1^{+}\right\rangle^{2}}{\left\langle 1^{-}\left|\not k_{j_{2}} K_{i_{1} \ldots i_{2}}\right| 1^{+}\right\rangle}\right. \\
\left.+\left\langle 1^{-}\left|K_{a \ldots b} \not k_{j_{1}}\right| 1^{+}\right\rangle \pm\left\langle 1^{-}\left|\not K_{a \ldots b} \not k_{j_{2}}\right| 1^{+}\right\rangle\right) .
\end{array}
$$

\section{CONCLUSIONS}

At the LHC, events involving large numbers of jets will play a central role in investigations and measurements of new physics. On the theoretical side, a proper understanding of such events will require NLO calculations. These calculations in turn require one-loop amplitudes with large numbers of hard colored final-state particles.

In this paper we have provided new and non-trivial examples of one-loop QCD amplitudes with an arbitrary number of external legs. We have computed these amplitudes using the on-shell bootstrap method [31, 32]. Previously, $n$-gluon amplitudes with special helicity configurations, with two or three color-adjacent negative-helicity legs, have been computed $[32,47]$. In the computation of three color-adjacent negative-helicity legs, several theoretical issues mentioned in the introduction - non-standard channels and large 
shift-parameter behavior — arose and were resolved in a general manner. The calculation presented here is in these respects simpler, in that our choice of shift eliminates these issues. Here we have extended the results to cover all one-loop corrections to the celebrated MHV amplitudes of Parke and Taylor [62]. These are amplitudes with the two negative helicities in arbitrary positions in the color ordering. These amplitudes are most conveniently expressed in a supersymmetric decomposition [7,26], for which many of the ingredients had been computed previously in refs. $[6,7,18]$. The ensemble of terms, including the rational parts of the scalar-loop contributions given here, provide an expression for this class of amplitudes that is compact when compared to expectations based on a brute-force diagrammatic calculation.

These results will be of direct use in studies of multi-jet physics at the LHC. They also allow us to confirm the relatively mild increase in complexity of our methods as the number of external legs increases. For many years, it has been widely believed that the factorial growth in the complexity of gauge theory calculations is an intrinsic part of the perturbative expansion. Our construction illustrates in a non-trivial context that this is not so.

In constructing the amplitudes we made use of a number of empirically-observed properties. In the calculations described in this paper, we have confirmed the validity of these assumptions using the stringent requirements of proper symmetries and factorization in all channels under real momenta. For six gluons, we also confirmed agreement with the numerical results of Ellis, Giele and Zanderighi [4] and also with the analytic results of Xiao, Yang and Zhu [52].

Although analytic results for the rational parts of the two remaining, $\mathcal{N}=0$ non-MHV six-gluon amplitudes have been obtained recently [52], it is still of interest to use the recursive bootstrap to construct them from the known cut-containing parts [25]. One reason to do so is in order to rearrange the rational parts, so that their spurious singularities are more manifestly cancelled against those of the cut parts. A second reason would be to see whether a more compact form can be obtained, in the interest of faster numerical evaluation of the amplitude.

We may contrast the speed of numerical evaluation using our expressions with that of the semi-numerical approach of refs. [2,3]. The semi-numerical computation of the complete six-gluon amplitude takes 9 seconds to evaluate one $\mathcal{N}=0$ helicity configuration on a 2.8 GHz Pentium processor [4]. There are 64 helicity configurations in total. (Subsequent helicity configurations will not take as long as 9 seconds, though.) We have implemented the 
$\mathcal{N}=4, \mathcal{N}=1$ and $\mathcal{N}=0$ components of the six-gluon amplitude in $\mathrm{C}++$, except for the $\mathcal{N}=0$ components of the non-MHV helicity configurations $-(-+-+-+),(--+-++)$ and permutations (14 in all, out of 64 ). The terms we have implemented so far require 30 milliseconds in total to evaluate to 9 significant digits on a $2 \mathrm{GHz}$ Xeon processor, for all 64 helicity configurations, which is considerably faster than the semi-numerical approach.

There are a number of open issues that would be important to address. For example, it would be useful to have a first-principles derivation of the complex factorization properties, as well as of the behavior of loop amplitudes at large values of the shift parameter. In this regard, recent papers [63] linking tree-level on-shell recursion with gauge-theory Lagrangians in particular gauges may prove useful. The unitarity method with $D$-dimensional cuts [8] may also assist in the formal understanding of properties of gauge-theory amplitudes.

It would also be very important to apply the on-shell bootstrap to processes involving external vector bosons and quarks. (For it to be applicable to cases with massive quarks, one would need to first extend the methods to allow massive particles in the loop.) Such processes are of crucial importance for understanding backgrounds to new physics in supersymmetric and other extensions of the Standard Model.

With a set of one-loop multi-parton matrix elements in hand, one can proceed to construct a numerical program for NLO differential cross sections. Although the construction of such programs is non-trivial, it is well-understood, and very general formalisms are known [64].

Another interesting open problem concerns the twistor-space properties [37] of the loop amplitudes. There have already been some studies of these properties [65-67]. In particular, the coefficients of box integrals have a surprisingly simple twistor-space structure, exhibiting delta-function support on intersecting lines, as described in some detail for the case of $\mathcal{N}=4$ super-Yang-Mills theory [20, 23, 34, 68]. It would be interesting to map out the twistor-space properties of the complete amplitudes, especially in non-supersymmetric theories, using the all- $n$ expressions obtained here and elsewhere [7, 18, 31, 32, 46, 58, 59]. Using the twistorspace structure as a guide, it may, for example, be possible to construct a set of loop-level MHV vertices, incorporating both rational and cut terms, in analogy with the tree-level construction of amplitudes with generic helicities using MHV vertices [15].

It might also be possible to use the one-loop MHV amplitudes to obtain insight into the size of NLO corrections to hadron collider processes with a large number of jets. At tree level, one of the early applications of the MHV (Parke-Taylor) amplitudes [62, 69, 70] 
was as the basis of schemes for estimating multi-jet rates at hadron colliders, in advance of the availability of exact matrix elements for all helicity configurations. One approach was to simply assume that the non-MHV amplitudes were the same as the MHV amplitudes, and multiply the MHV terms in the cross section by a simple combinatoric factor [71]. Subsequently, a procedure known as 'infrared reduction', designed to match the known collinear behavior, was applied to the case of four-jet production, and gave results quite similar to the exact matrix elements [72]. It would be interesting to see whether similar procedures could be applied sensibly to NLO computations as well, making use of the MHV loop amplitudes reported here, as well as similar ones containing quarks, should they become available.

We anticipate that the on-shell unitarity-bootstrap approach will have widespread applications to computing the higher-multiplicity amplitudes required for next-to-leading order computations of phenomenological interest at the Large Hadron Collider.

\section{Acknowledgments}

We thank Xun Su, Zhi-Guang Xiao, Gang Yang and Chuan-Jie Zhu for sending us their results prior to publication [51, 52], and for discussions and assistance in comparing our sixpoint results with theirs. We thank Academic Technology Services at UCLA for computer support. The figures were generated using Jaxodraw [73], based on Axodraw [74].

\section{APPENDIX A: COMPLETED-CUT TERMS OF $\mathcal{N}=0$ MHV AMPLITUDES}

In this appendix we collect the cut parts for the $\mathcal{N}=0$ one-loop amplitudes with two negative-helicity gluons, which were obtained in ref. [18], and complete them in a convenient way. We also give the rational parts of these completed-cut terms in a form convenient for computing the overlap terms. Converting the expressions in ref. [18] to a notation similar to that used for the $\mathcal{N}=1$ amplitudes in ref. [7], and adding suitable rational terms via $\mathrm{L}_{i}$ functions, we have the completed-cut parts of the MHV amplitude,

$$
\begin{array}{r}
\widehat{C}_{n}^{\mathcal{N}=0}(1, m)= \\
A_{n}^{\text {tree }}(1, m)
\end{array}
$$




$$
\begin{aligned}
& \times\left\{\sum_{j_{1}=2}^{m-1} \sum_{j_{2}=m+1}^{n}-\frac{1}{2}\left[b_{j_{1}, j_{2}}^{m}\right]^{2} \mathrm{M}_{0}\left(s_{\left(j_{1}+1\right) \ldots j_{2}}, s_{j_{1} \ldots\left(j_{2}-1\right)} ; s_{\left(j_{1}+1\right) \ldots\left(j_{2}-1\right)}, s_{\left(j_{2}+1\right) \ldots\left(j_{1}-1\right)}\right)\right. \\
& +\sum_{2 \leq j<m} \sum_{a \in \hat{\chi}_{j}}\left[d_{j, a}^{m} \frac{\mathrm{L}_{2}\left(\frac{-s_{(j+1) \ldots a}}{-s_{j \ldots a}}\right)}{s_{j \ldots a}^{3}}+e_{j, a}^{m} \frac{\widehat{\mathrm{L}}_{1}\left(\frac{-s_{(j+1) \ldots a}}{-s_{j \ldots a}}\right)}{s_{j \ldots a}^{2}}\right. \\
& \left.+\left(\frac{1}{6} c_{j, a}^{m}+f_{j, a}^{m}\right) \frac{\mathrm{L}_{0}\left(\frac{-s_{(j+1) \ldots a}}{-s_{j \ldots a}}\right)}{s_{j \ldots a}}-\frac{1}{4}\left(b_{j, a}^{m}-b_{j, a+1}^{m}\right) \ln \left(\frac{-s_{(j+1) \ldots a}}{-s_{j \ldots a}}\right)\right] \\
& +\sum_{m<j \leq n} \sum_{a \in \chi_{j}}\left[d_{j, a}^{m} \frac{\mathrm{L}_{2}\left(\frac{-s_{(a+1) \ldots j}}{-s_{(a+1) \ldots(j-1)}}\right)}{s_{(a+1) \ldots(j-1)}^{3}}+e_{j, a}^{m} \frac{\widehat{\mathrm{L}}_{1}\left(\frac{-s_{(a+1) \ldots j}}{-s_{(a+1) \ldots(j-1)}}\right)}{s_{(a+1) \ldots(j-1)}^{2}}\right. \\
& +\left(\frac{1}{6} c_{j, a}^{m}+f_{j, a}^{m}\right) \frac{\mathrm{L}_{0}\left(\frac{-s_{(a+1) \ldots j}}{-s_{(a+1) \ldots(j-1)}}\right)}{s_{(a+1) \ldots(j-1)}} \\
& \left.-\frac{1}{4}\left(b_{j, a}^{m}-b_{j, a+1}^{m}\right) \ln \left(\frac{-s_{(a+1) \ldots j}}{-s_{(a+1) \ldots(j-1)}}\right)\right] \\
& +\left(\frac{1}{6} \frac{c_{2, n}^{m}}{s_{12}}+\frac{f_{2, n}^{m}}{s_{12}}+\frac{1}{4} b_{2, n}^{m}\right) \mathrm{K}_{0}\left(s_{12}\right)+\left(\frac{1}{6} \frac{c_{n, 1}^{m}}{s_{n 1}}+\frac{f_{n, 1}^{m}}{s_{n 1}}+\frac{1}{4} b_{n, 2}^{m}\right) \mathrm{K}_{0}\left(s_{n 1}\right) \\
& +\left(\frac{1}{6} \frac{c_{m+1, m-1}^{m}}{s_{m(m+1)}}+\frac{f_{m+1, m-1}^{m}}{s_{m(m+1)}}+\frac{1}{4} b_{m+1, m-1}^{m}\right) \mathrm{K}_{0}\left(s_{m(m+1)}\right) \\
& \left.+\left(\frac{1}{6} \frac{c_{m-1, m}^{m}}{s_{(m-1) m}}+\frac{f_{m-1, m}^{m}}{s_{(m-1) m}}+\frac{1}{4} b_{m-1, m+1}^{m}\right) \mathrm{K}_{0}\left(s_{(m-1) m}\right)\right\} .
\end{aligned}
$$

In performing the cut completion, we have rearranged the cuts somewhat before introducing the $\mathrm{L}_{i}$ functions, which automatically remove the spurious singularities. The coefficients appearing in the cuts are,

$$
\begin{aligned}
b_{j_{1}, j_{2}}^{m}= & 2 \frac{\left\langle 1 j_{1}\right\rangle\left\langle 1 j_{2}\right\rangle\left\langle m j_{1}\right\rangle\left\langle m j_{2}\right\rangle}{\langle 1 m\rangle^{2}\left\langle j_{1} j_{2}\right\rangle^{2}} \\
c_{j, a}^{m}= & \frac{\langle m j\rangle\left\langle j^{+}\left|K_{j \ldots a}\right| 1^{+}\right\rangle-\left\langle m^{-}\left|K_{j \ldots a}\right| j^{-}\right\rangle\langle j 1\rangle}{\langle 1 m\rangle^{2}}\langle j 1\rangle\langle m j\rangle \frac{\langle a(a+1)\rangle}{\langle a j\rangle\langle j(a+1)\rangle} \\
d_{j, a}^{m}= & -\frac{1}{3} c_{j, a}^{m} \frac{\langle 1 j\rangle\langle m j\rangle\left\langle 1^{-}\left|K_{(j+1) \ldots a}\right| j^{-}\right\rangle\left\langle m^{-}\left|K_{(j+1) \ldots a}\right| j^{-}\right\rangle}{\langle 1 m\rangle^{2}} \\
e_{j, a}^{m}= & -\frac{1}{2} \frac{\langle 1 j\rangle\langle m j\rangle}{\langle 1 m\rangle^{2}}\left\langle 1^{-}\left|K_{(j+1) \ldots a}\right| j^{-}\right\rangle\left\langle m^{-}\left|K_{(j+1) \ldots a}\right| j^{-}\right\rangle\left(b_{j, a}^{m}-b_{j, a+1}^{m}\right) \\
f_{j, a}^{m}= & \left(\frac{\langle 1 j\rangle\langle m j\rangle}{\langle 1 m\rangle^{2}}\right)^{2}\left[\frac{\langle 1 a\rangle\langle m a\rangle}{\langle a j\rangle^{3}}\left(\langle 1 a\rangle\left\langle m^{-}\left|K_{(j+1) \ldots a}\right| j^{-}\right\rangle+\langle m a\rangle\left\langle 1^{-}\left|K_{(j+1) \ldots a}\right| j^{-}\right\rangle\right)\right. \\
& -\frac{\langle 1(a+1)\rangle\langle m(a+1)\rangle}{\langle(a+1) j\rangle^{3}}\left(\langle 1(a+1)\rangle\left\langle m^{-}\left|K_{(j+1) \ldots a}\right| j^{-}\right\rangle\right. \\
& \left.\left.+\langle m(a+1)\rangle\left\langle 1^{-}\left|K_{(j+1) \ldots a}\right| j^{-}\right\rangle\right)\right]
\end{aligned}
$$




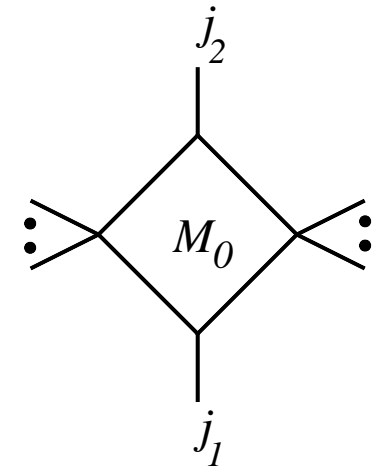

(a)

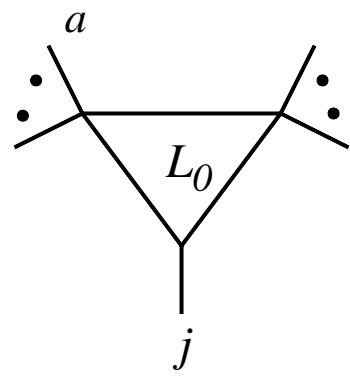

(b)

FIG. 11: Kinematic configuration of the box and triangle functions appearing in the completed-cut contribution given in eq. (A1).

The quantities $b_{j_{1}, j_{2}}^{m}$ and $c_{j, a}^{m}$ also appear in the expression for the $\mathcal{N}=1$ supersymmetric amplitude [7].

The sums over the $\mathrm{L}_{i}$ triangle functions run over the ranges corresponding to all configurations in fig. 11(b) with two massive corners, each containing a negative-helicity leg,

$$
\begin{aligned}
& \chi_{j}= \begin{cases}\{1,2, \ldots, m-2\}, & j=m+1, \\
\{1,2, \ldots, m-1\}, & m+1<j<n, \\
\{2, \ldots, m-1\}, & j=n,\end{cases} \\
& \hat{\chi}_{j}= \begin{cases}\{m, m+1, \ldots, n-1\}, \quad j=2, \\
\{m, m+1, \ldots, n\}, & 2<j<m-1, \\
\{m+1, m+2, \ldots, n\}, & j=m-1 .\end{cases}
\end{aligned}
$$

For $\chi_{j}$ when $m=n-1$ and $j=n$, and for $\hat{\chi}_{j}$ when $m=3$ and $j=2$, the kinematics degenerates and the intersection of the first and third conditions in eqs. (A7) and (A8) should be used. The $\mathrm{K}_{0}$ functions correspond to cases where the two-external-mass triangles degenerate to bubbles. For adjacent negative helicities, there are no boxes, and the remaining double sum collapses to a single sum, leaving the simpler expression given in ref. [7].

The box function is defined by

$$
\begin{gathered}
\mathrm{M}_{0}\left(s_{1}, s_{2} ; P^{2}, Q^{2}\right)=\mathrm{Li}_{2}\left(1-\frac{P^{2} Q^{2}}{s_{1} s_{2}}\right)-\mathrm{Li}_{2}\left(1-\frac{P^{2}}{s_{1}}\right)-\mathrm{Li}_{2}\left(1-\frac{P^{2}}{s_{2}}\right)-\mathrm{Li}_{2}\left(1-\frac{Q^{2}}{s_{1}}\right) \\
-\mathrm{Li}_{2}\left(1-\frac{Q^{2}}{s_{2}}\right)-\frac{1}{2} \ln ^{2}\left(\frac{s_{1}}{s_{2}}\right) .
\end{gathered}
$$


The box function is equal to minus the two-mass easy box function of ref. [21], $\mathrm{M}_{0}\left(s, t, P^{2}, Q^{2}\right)=-\mathrm{Ls}_{-1}^{2 m e}\left(s, t, P^{2}, Q^{2}\right)$, corresponding to a $D=6$ box integral. An alternative expression for this box integral may be found in ref. [17].

The $\mathrm{L}_{i}$ functions [26] are given in eq. (4.6). In the above we have replaced $\mathrm{L}_{1}$ with a somewhat different function, modified so as to better respect the symmetry properties of the amplitude,

$$
\widehat{\mathrm{L}}_{1}(r)=\frac{\ln (r)-(r-1 / r) / 2}{(1-r)^{2}} .
$$

In addition, we have the bubble function,

$$
\mathrm{K}_{0}(s)=\frac{1}{\epsilon(1-2 \epsilon)}\left(-s / \mu^{2}\right)^{-\epsilon}=\left(-\ln \left(-s / \mu^{2}\right)+2+\frac{1}{\epsilon}\right)+\mathcal{O}(\epsilon) .
$$

By using the $\widehat{L}_{1}$ and $\mathrm{L}_{2}$ functions in the completed-cut expression (A1) instead of logarithms, we eliminate all of the spurious poles in $\widehat{C}_{n}^{\mathcal{N}=0}$ that develop $z$ dependence under the $[1, m\rangle$ shift. These poles arise from differences of four-momentum invariants, for example $\left(s_{(j+1) \ldots a}-s_{j \ldots a}\right)^{3}$ in the denominator of $\mathrm{L}_{2}\left(\left(-s_{(j+1) \ldots a}\right) /\left(-s_{j \ldots a}\right)\right)$. These invariants contain leg $m$ but not leg 1 , so they are each shifted, leading to a $z$-dependent denominator.

Note that there are a host of other spurious poles in eq. (A1) that do not develop dependence on $z$ under the $[1, m\rangle$ shift. We do not need to worry about cancelling their spurious behavior; it will happen automatically using the rational terms we construct. We discussed this issue for the five-gluon amplitude in section IV A. In the $n$-gluon case it is a bit more intricate. The denominator factors of $\langle 1 m\rangle$ are cancelled by a numerator factor of $\langle 1 m\rangle^{4}$ in $A_{n}^{\text {tree }}(1, m)$, but in any case $\langle 1 m\rangle$ is left invariant by the $[1, m\rangle$ shift, so no $z$-dependent pole is induced. Now, the denominator factors of $\left\langle j_{1} j_{2}\right\rangle$ in $b_{j_{1}, j_{2}}^{m}$ are not entirely cancelled by numerator factors (the function $\mathrm{M}_{0}$ does cancel some of their singular behavior); but again, because neither $j_{1}$ nor $j_{2}$ can equal 1 or $m$, these factors are left invariant by the $[1, m\rangle$ shift. Finally, there are denominator factors of $\langle a j\rangle$ and $\langle(a+1) j\rangle$ in $c_{j, a}^{m}, e_{j, a}^{m}$ and $f_{j, a}^{m}$. While $j$ cannot be equal to 1 or $m, a$ or $(a+1)$ can be. However, the relevant terms are protected from $z$-dependent spurious poles by numerator factors of $\langle 1 j\rangle,\langle m j\rangle,\langle m a\rangle$ or $\langle m(a+1)\rangle$.

Since the entire amplitude must be free of spurious poles, we can now conclude that the rational remainder we wish to compute is free of $z$-dependent spurious poles.

The rational parts of the completed-cut terms are obtained by setting all logarithms, 
polylogarithms and $\pi^{2}$ terms to zero in eq. (A1),

$$
\widehat{C R}_{n}(1, m)=\left.\widehat{C}_{n}^{\mathcal{N}=0}(1, m)\right|_{\ln , \mathrm{Li}_{2}, \pi^{2} \rightarrow 0} .
$$

After some rearrangement, this expression can be written as,

$$
\begin{aligned}
& \widehat{C R}_{n}(1, m)=A_{n}^{\text {tree }}(1, m) {\left[\frac{1}{3}\left(\frac{1}{\epsilon}+2\right)\right.} \\
&+\frac{1}{2} \sum_{j_{1}=1}^{m-1} \sum_{j_{2}=\max \left(m, j_{1}+2\right)}^{\min \left(n, j_{1}+n-2\right)} \sum_{r=2}^{3} \frac{1}{s_{\left(j_{1}+1\right) \ldots j_{2}}} \\
& \times\left(\mathcal{N}_{+; j_{1} j_{2}}\left(r, j_{1}, j_{2}+1\right)+\mathcal{N}_{-; j_{1} j_{2}}\left(r, j_{1}+1, j_{2}+1\right)\right. \\
&\left.\left.\quad-\mathcal{N}_{+; j_{1} j_{2}}\left(r, j_{1}+1, j_{2}\right)-\mathcal{N}_{-; j_{1} j_{2}}\left(r, j_{1}, j_{2}\right)\right)\right]
\end{aligned}
$$

where

$$
\mathcal{N}_{ \pm ; j_{1} j_{2}}(r, a, b)=N_{r ; j_{1} j_{2}}(a, b, m, 1) \pm N_{r ; j_{1} j_{2}}(b, a, 1, m)
$$

with

$$
\begin{aligned}
& N_{2 ; j_{1} j_{2}}\left(a, b, m_{1}, m_{2}\right)=\frac{S_{j_{1} j_{2}}\left(a, b, m_{1}, m_{2}\right)}{\left\langle a^{-}\left|K_{\left(j_{1}+1\right) \ldots j_{2}}\right| a^{-}\right\rangle} \\
& N_{3 ; j_{1} j_{2}}\left(a, b, m_{1}, m_{2}\right)=\frac{C_{j_{1} j_{2}}\left(a, b, m_{1}, m_{2}\right)}{\left\langle a^{-}\left|K_{\left(j_{1}+1\right) \ldots j_{2}}\right| a^{-}\right\rangle^{2}} .
\end{aligned}
$$

These quantities in turn depend upon,

$$
\begin{aligned}
& C_{j_{1} j_{2}}\left(a, b, m_{1}, m_{2}\right)= \\
& -\left\langle m_{1} a\right\rangle\left\langle m_{2} b\right\rangle\left\langle m_{2}{ }^{-}\left|\not k_{a} \not K_{\left(j_{1}+1\right) \ldots j_{2}}\right| m_{1}{ }^{+}\right\rangle \\
& \quad \times \frac{\left\langle m_{2}{ }^{-}\left|K_{\left(j_{1}+1\right) \ldots j_{2}} \not k_{a}\right| m_{1}{ }^{+}\right\rangle\left\langle m_{2}{ }^{-}\left|k_{a} \not K_{\left(j_{1}+1\right) \ldots j_{2}}-\not K_{\left(j_{1}+1\right) \ldots j_{2}} \not k_{a}\right| m_{1}{ }^{+}\right\rangle}{3\left\langle m_{1} m_{2}\right\rangle^{4}\langle a b\rangle}, \\
& S_{j_{1} j_{2}}\left(a, b, m_{1}, m_{2}\right)= \\
& \left\langle m_{1} a\right\rangle\left\langle m_{2} b\right\rangle\left\langle m_{2} a\right\rangle\left\langle m_{1} b\right\rangle \\
& \quad \times \frac{\left\langle m_{2}{ }^{-}\left|K_{\left(j_{1}+1\right) \ldots j_{2}} k_{a}\right| m_{1}{ }^{+}\right\rangle\left\langle m_{1}{ }^{-}\left|K_{\left(j_{1}+1\right) \ldots j_{2}} k_{a}\right| m_{2}{ }^{+}\right\rangle}{\left\langle m_{1} m_{2}\right\rangle^{4}\langle a b\rangle^{2}} .
\end{aligned}
$$

Applying the $[1, m\rangle$ shift to $\widehat{C}_{n}^{\mathcal{N}=0}(1, m)$ in eq. (A1) and taking the $z \rightarrow \infty$ limit, we can extract the value of $\operatorname{Inf} \widehat{C}_{n}$ required by the basic formula (3.23). The result is,

$$
\begin{aligned}
& \operatorname{Inf} \widehat{C}_{n}(1, m)=A_{n}^{\text {tree }}(1, m) \\
& \quad \times \sum_{j_{1}=2}^{m-1} \sum_{j_{2}=m+1}^{n} \frac{\langle(m-1) m\rangle\langle m(m+1)\rangle}{\langle(m-1) 1\rangle\langle 1 m\rangle^{2}\langle 1(m+1)\rangle}\left(\frac{\left\langle 1 j_{1}\right\rangle^{3}\left\langle 1 j_{2}\right\rangle^{3}\left[j_{2} j_{1}\right]^{2}}{\left\langle j_{1} j_{2}\right\rangle^{2}\left\langle j_{1}{ }^{+}\left|k_{m}\right| 1^{+}\right\rangle\left\langle j_{2}{ }^{+}\left|k_{m}\right| 1^{+}\right\rangle}\right. \\
& \left.\quad-R_{+; j_{1} j_{2}}^{m m}\left(j_{1}, j_{2}\right)+R_{+; j_{1} j_{2}}^{m m}\left(j_{1}+1, j_{2}-1\right)+R_{-; j_{1} j_{2}}^{m m}\left(j_{1}, j_{2}-1\right)-R_{-; j_{1} j_{2}}^{m m}\left(j_{1}+1, j_{2}\right)\right),
\end{aligned}
$$


where we set $R_{ \pm ; j_{1} j_{2}}^{m m}(a, b)=0$ if $K_{a \ldots b}^{2}=0$; otherwise $R_{ \pm ; j_{1} j_{2}}^{m m}(a, b)$ is,

$$
\begin{array}{r}
R_{ \pm ; j_{1}, j_{2}}^{m m}(a, b)=\frac{\left\langle 1 j_{1}\right\rangle^{2}\left\langle 1 j_{2}\right\rangle^{2}}{2\left\langle j_{1} j_{2}\right\rangle^{2}\left\langle 1^{-}\left|K_{a \ldots b} \not k_{m}\right| 1^{+}\right\rangle}\left(\frac{\left\langle 1^{-}\left|\not K_{a \ldots b} \not k_{j_{1}}\right| 1^{+}\right\rangle^{2}}{\left\langle 1^{-}\left|k_{j_{1}} k_{m}\right| 1^{+}\right\rangle} \pm \frac{\left\langle 1^{-}\left|\not K_{a \ldots b} \not k_{j_{2}}\right| 1^{+}\right\rangle^{2}}{\left\langle 1^{-}\left|\not k_{j_{2}} \not k_{m}\right| 1^{+}\right\rangle}\right. \\
\left.+\left\langle 1^{-}\left|\not K_{a \ldots b} \not k_{j_{1}}\right| 1^{+}\right\rangle \pm\left\langle 1^{-}\left|\not K_{a \ldots b} \not k_{j_{2}}\right| 1^{+}\right\rangle\right) .
\end{array}
$$

Up to a factor of $-A_{n}^{\text {tree }}(1, m)$, this expression can also be obtained from formula (5.54) for $T_{7}^{i_{1} i_{2}}(n)$ by setting $i_{1}=i_{2}=m$.

\section{APPENDIX B: RATIONAL PARTS OF SIX-GLUON $\mathcal{N}=0$ MHV AMPLITUDES}

There are three independent six-gluon MHV amplitudes. All others can be obtained from these by cyclic permutations of the external legs. The analytic form of the $\mathcal{N}=0$ amplitude with adjacent negative helicities, $A_{6 ; 1}^{\mathcal{N}=0}(1,2)$, may be found in ref. [31]. In this appendix we present analytic forms for the rational remainders of the other two independent MHV amplitudes. Although the structure is rather intricate, such forms can be useful for future phenomenological studies of four-jet events. Given their mild spurious singularities (compared to more direct evaluations of Feynman diagrams), we do not anticipate any significant complications arising from round-off error when constructing an NLO program.

The amplitude $A_{6 ; 1}^{\mathcal{N}=0}(1,3)$ is given by

$$
A_{6 ; 1}^{\mathcal{N}=0}(1,3)=c_{\Gamma}\left[\widehat{C}_{6}(1,3)+\widehat{R}_{6}(1,3)\right]
$$

where $\widehat{C}_{6}(1,3)$ is given in eq. (A1),

$$
\widehat{R}_{6}(1,3)=-\operatorname{Inf} \widehat{C}_{6}(1,3)+\widehat{R}_{6}^{a}(1,3)
$$

and $\operatorname{Inf} \widehat{C}_{6}(1,3)$ is given in eq. (A17) with $n=6$ and $m=3$. After simplification, the result for $\widehat{R}_{6}^{a}(1,3)$ is,

$$
\begin{aligned}
\widehat{R}_{6}^{a} & (1,3) \\
& =i\left[-\frac{[24]^{3}}{3\langle 56\rangle^{2}[12][13][34]}-\frac{2\langle 13\rangle^{4}}{9\langle 12\rangle\langle 16\rangle\langle 23\rangle\langle 34\rangle\langle 45\rangle\langle 56\rangle}\right. \\
& +\frac{\langle 13\rangle^{2}\langle 14\rangle[24]}{6\langle 16\rangle\langle 23\rangle\langle 24\rangle\langle 45\rangle\langle 56\rangle[23]}+\frac{\left\langle 1^{-}|(2+5)| 4^{-}\right\rangle\langle 13\rangle^{2}\langle 15\rangle}{2\langle 14\rangle\langle 16\rangle\langle 25\rangle^{2}\langle 34\rangle\langle 56\rangle[34]} \\
& -\frac{\left\langle 1^{-}|(3+5)| 4^{-}\right\rangle^{3}\left\langle 1^{-}|(4+5)| 3^{-}\right\rangle[35]}{3\left\langle 2^{-}|(4+5)| 3^{-}\right\rangle\left\langle 6^{-}|(1+2)| 3^{-}\right\rangle\langle 12\rangle\langle 16\rangle\langle 45\rangle[34]^{2} s_{345}}
\end{aligned}
$$




$$
\begin{aligned}
& -\frac{[24]^{3}[36]^{3}}{3\left\langle 5^{-}|(2+4)| 3^{-}\right\rangle^{2}[13][16][23][34]}-\frac{\langle 13\rangle^{3}\langle 15\rangle[25]}{6\left\langle 1^{-}|(3+4)| 2^{-}\right\rangle\langle 16\rangle\langle 24\rangle\langle 25\rangle\langle 34\rangle\langle 56\rangle}
\end{aligned}
$$

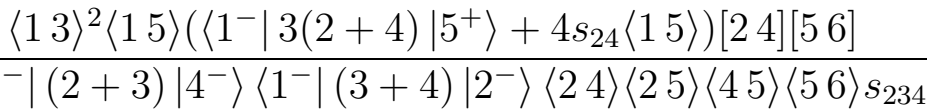

$$
\begin{aligned}
& -\frac{s_{26}\left\langle 1^{-}|(4+5)| 3^{-}\right\rangle^{3}[45]^{3}}{3\left\langle 1^{-}|(3+4)| 5^{-}\right\rangle\left\langle 2^{-}|4+5| 3^{-}\right\rangle^{2}\left\langle 6^{-}|(1+2)| 3^{-}\right\rangle^{2}[34] s_{345}} \\
& -\frac{\left\langle 5^{-}|(1+3)| 6^{-}\right\rangle[26] s_{245}^{2}}{3\left\langle 4^{-}|(1+3)| 6^{-}\right\rangle\left\langle 5^{-}|(2+4)| 3^{-}\right\rangle\langle 26\rangle\langle 45\rangle\langle 56\rangle[13][16]} \\
& -\frac{\langle 13\rangle^{3}\langle 46\rangle[26]}{6\left\langle 4^{-}|(1+3)| 2^{-}\right\rangle\langle 12\rangle\langle 26\rangle\langle 34\rangle\langle 45\rangle\langle 56\rangle}+\frac{\left\langle 1^{-}|(2+3)| 4^{-}\right\rangle^{2}\langle 12\rangle\langle 15\rangle[24]}{2\left\langle 1^{-}|(2+4)| 3^{-}\right\rangle\langle 16\rangle\langle 25\rangle^{2}\langle 56\rangle[34] s_{234}} \\
& +\frac{\langle 13\rangle^{3}\langle 15\rangle\langle 25\rangle[45][56]}{6\left\langle 1^{-}|(2+3)| 4^{-}\right\rangle\left\langle 2^{-}|(1+3)| 6^{-}\right\rangle\langle 16\rangle\langle 23\rangle\langle 24\rangle\langle 45\rangle\langle 56\rangle} \\
& +\frac{\left\langle 1^{-}|(3+5)| 4^{-}\right\rangle\left(\langle 15\rangle[54]+\left\langle 1^{-}|(3+5)| 4^{-}\right\rangle\right)\langle 15\rangle^{2}[45]}{6\langle 12\rangle\langle 16\rangle\langle 25\rangle\langle 45\rangle\langle 56\rangle[34]^{2} s_{345}} \\
& -\frac{\left\langle 1^{-}|(3+5)| 4^{-}\right\rangle^{2}\langle 15\rangle^{2}[45]}{2\left\langle 1^{-}|(4+5)| 3^{-}\right\rangle\langle 16\rangle\langle 25\rangle^{2}\langle 56\rangle[34] s_{345}}-\frac{\left\langle 5^{-}|(1+3)| 2^{-}\right\rangle^{3}\langle 46\rangle[45]}{3\left\langle 6^{-}|(1+2)| 3^{-}\right\rangle\langle 45\rangle^{2}\langle 56\rangle^{2}[12][13] s_{123}} \\
& -\frac{(2\langle 12\rangle[24]+\langle 13\rangle[34])\left\langle 1^{-}|(2+3)| 4^{-}\right\rangle\langle 12\rangle[24]}{6\langle 16\rangle\langle 24\rangle\langle 25\rangle\langle 56\rangle[34]^{2} s_{234}} \\
& +\frac{\langle 13\rangle^{2}\left(\langle 12\rangle^{2}\langle 45\rangle[24]-\langle 15\rangle^{2}\langle 24\rangle[45]\right)}{6\langle 12\rangle\langle 16\rangle\langle 24\rangle\langle 25\rangle\langle 34\rangle\langle 45\rangle\langle 56\rangle[34]} \\
& -\frac{(\langle 13\rangle[32]+2\langle 14\rangle[42])\left\langle 1^{-}|(3+4)| 2^{-}\right\rangle\langle 14\rangle[24]}{6\langle 16\rangle\langle 24\rangle\langle 45\rangle\langle 56\rangle[23]^{2} s_{234}} \\
& +\frac{\left(s_{45}+s_{56}\right)\langle 13\rangle^{3}[46]^{2}}{3\left\langle 1^{-}|(2+3)| 4^{-}\right\rangle\left\langle 2^{-}|(1+3)| 6^{-}\right\rangle\langle 23\rangle\langle 45\rangle\langle 56\rangle s_{123}} \\
& -\frac{\left\langle 1^{-}|(2+4)| 3^{-}\right\rangle^{3}[24]^{3}}{3\left\langle 5^{-}|(2+4)| 3^{-}\right\rangle\langle 16\rangle\langle 24\rangle\langle 56\rangle[23]^{2}[34]^{2} s_{234}} \\
& +\frac{\left\langle 6^{-}|(1+3)| 2^{-}\right\rangle^{2}[26] s_{123}}{3\left\langle 4^{-}|(1+3)| 2^{-}\right\rangle\langle 26\rangle^{2}\langle 45\rangle\langle 56\rangle[12][13][23]} \\
& +\frac{\left\langle 5^{-}|(1+3)| 2^{-}\right\rangle\left\langle 6^{-}|(1+3)| 2^{-}\right\rangle^{2}[26]}{3\left\langle 4^{-}|(1+3)| 2^{-}\right\rangle\left\langle 6^{-}|(1+2)| 3^{-}\right\rangle\langle 45\rangle\langle 56\rangle^{2}[12][13]} \\
& -\frac{\left(s_{12}+s_{23}\right)\left\langle 6^{-}|(1+3)| 2^{-}\right\rangle\langle 16\rangle^{2}[26] s_{123}}{6\left\langle 4^{-}|(1+3)| 2^{-}\right\rangle\left\langle 6^{-}|(1+2)| 3^{-}\right\rangle\langle 12\rangle\langle 26\rangle^{2}\langle 45\rangle\langle 56\rangle[12][23]} \\
& \left\langle 1^{-}|(5+6)(2+4)| 5^{+}\right\rangle^{2}\langle 15\rangle^{2}[24][56] \\
& -\frac{\left\langle 1^{-}|(2+4)| 3^{-}\right\rangle\left\langle 1^{-}|(3+4)| 2^{-}\right\rangle\left\langle 5^{-}|(2+4)| 3^{-}\right\rangle\langle 25\rangle^{2}\langle 45\rangle\langle 56\rangle s_{234}}{2\left\langle 1^{-}|(3+4)| 2^{-}\right\rangle\left\langle 5^{-}|(1+3)| 6^{-}\right\rangle\langle 15\rangle\left[36 s_{24}\right.} \\
& +\frac{\left\langle 1^{-}|(3+4)| 2^{-}\right\rangle\left\langle 5^{-}|(1+3)| 6^{-}\right\rangle\langle 15\rangle[36] s_{245}^{2}}{3\left\langle 2^{-}|(4+5)| 3^{-}\right\rangle\left\langle 4^{-}|(1+3)| 6^{-}\right\rangle\left\langle 5^{-}|(2+4)| 3^{-}\right\rangle^{2}\langle 16\rangle\langle 45\rangle\langle 56\rangle[16]} \\
& +\frac{\left\langle 5^{-}|(1+3)| 6^{-}\right\rangle\langle 13\rangle[25][36]\left(\left\langle 5^{-}|(2+4)| 3^{-}\right\rangle\langle 13\rangle+2\langle 15\rangle s_{245}\right)}{3\left\langle 2^{-}|(4+5)| 3^{-}\right\rangle\left\langle 4^{-}|(1+3)| 6^{-}\right\rangle\left\langle 5^{-}|(2+4)| 3^{-}\right\rangle\langle 16\rangle\langle 45\rangle\langle 56\rangle[16]}
\end{aligned}
$$




$$
\begin{aligned}
& \left\langle 1^{-}|(2+6)(4+5)| 6^{+}\right\rangle^{2}\langle 12\rangle\langle 16\rangle[26][45] \\
& -\overline{2\left\langle 1^{-}|(3+4)| 5^{-}\right\rangle\left\langle 1^{-}|(4+5)| 3^{-}\right\rangle\left\langle 6^{-}|(1+2)| 3^{-}\right\rangle\langle 26\rangle^{2}\langle 45\rangle\langle 56\rangle s_{345}} \\
& +\frac{\left\langle 1^{-}|(2+6)(4+5)| 6^{+}\right\rangle\langle 16\rangle\left(\left\langle 1^{-}|3(4+5)| 6^{+}\right\rangle+2 s_{45}\langle 16\rangle\right)[26][45]}{6\left\langle 1^{-}|(3+4)| 5^{-}\right\rangle\left\langle 6^{-}|(1+2)| 3^{-}\right\rangle^{2}\langle 26\rangle\langle 45\rangle\langle 56\rangle s_{345}} \\
& -\frac{\left\langle 1^{-}|(2+6)(4+5)| 2^{+}\right\rangle\langle 12\rangle\left(\left\langle 1^{-}|3(4+5)| 2^{+}\right\rangle+2 s_{45}\langle 12\rangle\right)[26][45]}{6\left\langle 1^{-}|(3+4)| 5^{-}\right\rangle\left\langle 2^{-}|(4+5)| 3^{-}\right\rangle^{2}\langle 25\rangle\langle 26\rangle\langle 45\rangle s_{345}} \\
& +\frac{\left\langle 1^{-}|(2+3)| 4^{-}\right\rangle\left\langle 1^{-}|(3+4)| 2^{-}\right\rangle[24]\left(-\langle 12\rangle^{2}\langle 45\rangle[23]+\langle 14\rangle^{2}\langle 25\rangle[34]\right)}{2\left\langle 1^{-}|(2+4)| 3^{-}\right\rangle\langle 16\rangle\langle 24\rangle\langle 25\rangle\langle 45\rangle\langle 56\rangle[23][34] s_{234}} \\
& +\frac{\langle 13\rangle^{3}\langle 46\rangle^{2}[26]\left(2\left\langle 1^{-}|2(5+6)| 4^{+}\right\rangle+\left\langle 1^{-}|65| 4^{+}\right\rangle+\langle 14\rangle\langle 26\rangle[26]\right)}{2\left\langle 1^{-}|(3+4)| 5^{-}\right\rangle\left\langle 4^{-}|(1+3)| 2^{-}\right\rangle\langle 14\rangle\langle 26\rangle^{2}\langle 34\rangle\langle 45\rangle^{2}\langle 56\rangle} \\
& -\frac{\left\langle 5^{-}|(1+3)| 6^{-}\right\rangle^{2}\langle 15\rangle\left(3\left\langle 2^{-}|(4+5)| 6^{-}\right\rangle\langle 15\rangle-\langle 13\rangle\langle 25\rangle[36]\right)[56] s_{245}}{6\left\langle 2^{-}|(4+5)| 6^{-}\right\rangle\left\langle 4^{-}|(1+3)| 6^{-}\right\rangle\left\langle 5^{-}|(2+4)| 3^{-}\right\rangle\langle 16\rangle\langle 25\rangle^{2}\langle 45\rangle\langle 56\rangle[16][36]} \\
& -\frac{\langle 13\rangle^{3}\langle 15\rangle\left(\langle 14\rangle\langle 15\rangle\langle 24\rangle\langle 25\rangle[25]^{2}-\left\langle 1^{-}|52| 4^{+}\right\rangle\langle 16\rangle\langle 45\rangle[56]\right)}{2\left\langle 1^{-}|(3+4)| 2^{-}\right\rangle\left\langle 4^{-}|(1+3)| 6^{-}\right\rangle\langle 14\rangle\langle 16\rangle^{2}\langle 24\rangle\langle 25\rangle^{2}\langle 34\rangle\langle 56\rangle} \\
& +\frac{\left\langle 1^{-}|(2+6)(4+5)| 2^{+}\right\rangle\langle 12\rangle[26][45]}{2\left\langle 1^{-}|(3+4)| 5^{-}\right\rangle\left\langle 1^{-}|(4+5)| 3^{-}\right\rangle\left\langle 2^{-}|(4+5)| 3^{-}\right\rangle\langle 25\rangle^{2}\langle 26\rangle^{2}\langle 45\rangle s_{345}} \\
& \times\left(\left\langle 1^{-}|(2+6)(4+5)| 2^{+}\right\rangle\langle 16\rangle\langle 25\rangle+\left\langle 1^{-}|(2+6) 4| 5^{+}\right\rangle\langle 12\rangle\langle 26\rangle\right) \\
& \left\langle 5^{-}|(1+3)| 6^{-}\right\rangle^{3} \\
& -\frac{}{3\left\langle 2^{-}|(4+5)| 3^{-}\right\rangle\left\langle 2^{-}|(4+5)| 6^{-}\right\rangle\left\langle 5^{-}|(2+4)| 3^{-}\right\rangle\langle 45\rangle\langle 56\rangle[16]} \\
& \times\left(\frac{\langle 46\rangle[36][45]}{\langle 45\rangle[13]}+\frac{\langle 12\rangle[56] s_{245}}{\left\langle 4^{-}|(1+3)| 6^{-}\right\rangle\langle 25\rangle}\right) \\
& +\frac{\langle 13\rangle^{3}\left(\langle 15\rangle\langle 26\rangle\langle 45\rangle[25](6\langle 15\rangle\langle 24\rangle+\langle 14\rangle\langle 25\rangle)+2\langle 14\rangle\langle 16\rangle\langle 25\rangle^{2}\langle 46\rangle[26]\right)}{6\left\langle 4^{-}|(1+3)| 6^{-}\right\rangle\langle 14\rangle\langle 16\rangle^{2}\langle 25\rangle^{2}\langle 26\rangle\langle 34\rangle\langle 45\rangle\langle 56\rangle} \\
& -\frac{\langle 15\rangle^{3}[24]^{2}[56]}{6\left\langle 1^{-}|(2+3)| 4^{-}\right\rangle\left\langle 1^{-}|(3+4)| 2^{-}\right\rangle\left\langle 5^{-}|(2+4)| 3^{-}\right\rangle\langle 25\rangle\langle 45\rangle\langle 56\rangle^{2}[23][34] s_{234}} \\
& \times\left(2\langle 64\rangle[43]\langle 14\rangle\langle 25\rangle[24]^{2}+2\langle 62\rangle[23]\langle 12\rangle\langle 45\rangle[24]^{2}-5 s_{24}\langle 13\rangle\langle 56\rangle[23][34]\right) \\
& +\frac{\left\langle 2^{-}|(1+3)| 6^{-}\right\rangle\langle 12\rangle[26] s_{245}}{6\left\langle 2^{-}|(4+5)| 3^{-}\right\rangle\left\langle 4^{-}|(1+3)| 6^{-}\right\rangle\langle 16\rangle\langle 25\rangle^{2}\langle 26\rangle^{2}\langle 45\rangle\langle 56\rangle[16][36]} \\
& \times\left(3\left\langle 6^{-}|(1+3)| 6^{-}\right\rangle\langle 16\rangle\langle 25\rangle^{2}-3\left\langle 5^{-}|(1+3)| 6^{-}\right\rangle\langle 15\rangle\langle 26\rangle^{2}\right. \\
& -\langle 13\rangle\langle 25\rangle\langle 26\rangle\langle 56\rangle[36]) \\
& -\frac{\langle 13\rangle^{3}}{3\left\langle 1^{-}|(3+4)| 5^{-}\right\rangle\left\langle 4^{-}|(1+3)| 6^{-}\right\rangle\langle 34\rangle\langle 56\rangle}\left(\frac{\langle 12\rangle\langle 15\rangle^{2}[25]^{3}}{\left\langle 1^{-}|(3+4)| 2^{-}\right\rangle\langle 16\rangle^{2}\langle 25\rangle}\right. \\
& \left.-\frac{\langle 14\rangle[25]^{2}[26]^{2}}{\left\langle 1^{-}|(3+4)| 2^{-}\right\rangle\left\langle 4^{-}|(1+3)| 2^{-}\right\rangle}+\frac{\langle 24\rangle\langle 46\rangle^{2}[26]^{3}}{\left\langle 4^{-}|(1+3)| 2^{-}\right\rangle\langle 26\rangle\langle 45\rangle^{2}}\right) \\
& {[26] s_{245}^{2}} \\
& \overline{3\left\langle 2^{-}|(4+5)| 3^{-}\right\rangle\left\langle 4^{-}|(1+3)| 6^{-}\right\rangle\left\langle 5^{-}|(2+4)| 3^{-}\right\rangle\langle 16\rangle\langle 25\rangle\langle 26\rangle\langle 45\rangle\langle 56\rangle[13][16]} \\
& \times\left(\left\langle 5^{-}|(1+3)| 6^{-}\right\rangle\langle 12\rangle[13](\langle 12\rangle\langle 56\rangle-\langle 16\rangle\langle 25\rangle)\right.
\end{aligned}
$$




$$
\begin{aligned}
& \left.-\left\langle 1^{-}|(3+6)(1+3)| 2^{+}\right\rangle\langle 25\rangle\langle 56\rangle[36]\right) \\
& +\frac{\langle 13\rangle^{3}}{6\left\langle 1^{-}|(3+4)| 5^{-}\right\rangle\langle 14\rangle\langle 16\rangle\langle 25\rangle^{2}\langle 26\rangle^{2}\langle 34\rangle\langle 45\rangle^{2}\langle 56\rangle} \\
& \times\left(6\langle 15\rangle^{2}\langle 24\rangle\langle 26\rangle^{2}\langle 45\rangle[25]-2\langle 14\rangle\langle 15\rangle\langle 25\rangle\langle 26\rangle^{2}\langle 45\rangle[25]\right. \\
& \left.+6\langle 16\rangle^{2}\langle 24\rangle\langle 25\rangle^{2}\langle 46\rangle[26]+5\langle 14\rangle\langle 16\rangle\langle 25\rangle^{2}\langle 26\rangle\langle 46\rangle[26]\right) \\
& +\frac{\langle 12\rangle\langle 13\rangle^{3}\langle 15\rangle^{2}\langle 24\rangle[25]^{2}}{\left\langle 1^{-}|(3+4)| 5^{-}\right\rangle\left\langle 4^{-}|(1+3)| 6^{-}\right\rangle\langle 14\rangle\langle 16\rangle^{2}\langle 25\rangle^{2}\langle 34\rangle\langle 56\rangle} \\
& -\frac{\langle 12\rangle\langle 13\rangle^{3}\langle 24\rangle^{2}[25][26]}{2\left\langle 1^{-}|(3+4)| 5^{-}\right\rangle\left\langle 4^{-}|(1+3)| 6^{-}\right\rangle\langle 14\rangle\langle 25\rangle\langle 26\rangle^{2}\langle 34\rangle\langle 45\rangle} \\
& +\frac{\langle 12\rangle\langle 13\rangle^{3}\langle 15\rangle\langle 24\rangle[25][26]}{2\left\langle 1^{-}|(3+4)| 5^{-}\right\rangle\left\langle 4^{-}|(1+3)| 6^{-}\right\rangle\langle 14\rangle\langle 16\rangle\langle 25\rangle^{2}\langle 34\rangle\langle 56\rangle} \\
& -\frac{\langle 13\rangle^{3}\langle 14\rangle[25][26]}{6\left\langle 1^{-}|(3+4)| 5^{-}\right\rangle\left\langle 4^{-}|(1+3)| 6^{-}\right\rangle\langle 16\rangle\langle 34\rangle\langle 45\rangle\langle 56\rangle} \\
& -\frac{\langle 13\rangle^{3}\langle 24\rangle[25][26]}{2\left\langle 1^{-}|(3+4)| 5^{-}\right\rangle\left\langle 4^{-}|(1+3)| 6^{-}\right\rangle\langle 26\rangle\langle 34\rangle\langle 45\rangle\langle 56\rangle} \\
& \left.+\frac{\langle 12\rangle^{2}\langle 13\rangle^{3}\langle 46\rangle[25][26]}{2\left\langle 1^{-}|(3+4)| 5^{-}\right\rangle\left\langle 4^{-}|(1+3)| 6^{-}\right\rangle\langle 14\rangle\langle 16\rangle\langle 25\rangle\langle 26\rangle\langle 34\rangle\langle 56\rangle}\right] .
\end{aligned}
$$

The amplitude $A_{6 ; 1}^{\mathcal{N}=0}(1,4)$ can be assembled analogously from eqs. (A1), (A17) and (B7),

$$
A_{6 ; 1}^{\mathcal{N}=0}(1,4)=c_{\Gamma}\left[\widehat{C}_{6}(1,4)+\widehat{R}_{6}(1,4)\right],
$$

where

$$
\widehat{R}_{6}(1,4)=-\operatorname{Inf} \widehat{C}_{6}(1,4)+\widehat{R}_{6}^{a}(1,4)+\left.\widehat{R}_{6}^{a}(1,4)\right|_{\text {flip }},
$$

and where we have used the flip symmetry,

$$
\left.X(1,2,3,4,5,6)\right|_{\text {flip }} \equiv X(1,6,5,4,3,2) .
$$

After simplification, we find,

$$
\begin{aligned}
\widehat{R}_{6}^{a}(1,4) & \\
\quad & i\left[-\frac{8\langle 13\rangle\langle 14\rangle^{3}}{9\langle 12\rangle\langle 16\rangle\langle 23\rangle\langle 34\rangle\langle 35\rangle\langle 56\rangle}-\frac{(\langle 14\rangle[43]+2\langle 15\rangle[53])\left\langle 1^{-}|(4+5)| 3^{-}\right\rangle\langle 15\rangle^{2}[35]}{6\langle 12\rangle\langle 16\rangle\langle 25\rangle\langle 35\rangle\langle 56\rangle[34]^{2} s_{345}}\right. \\
& +\frac{\left\langle 2^{-}|(1+4)| 6^{-}\right\rangle\left\langle 5^{-}|(1+4)| 6^{-}\right\rangle\langle 15\rangle^{2}[56] s_{146}}{2 s_{16}\left\langle 3^{-}|(1+4)| 6^{-}\right\rangle\left\langle 5^{-}|(2+3)| 4^{-}\right\rangle\langle 23\rangle\langle 25\rangle^{2}\langle 56\rangle[46]} \\
& -\frac{[35]^{3}[46]^{3}}{3\left\langle 2^{-}|(3+5)| 4^{-}\right\rangle^{2}[14][16][34][45]}+\frac{\left\langle 1^{-}|(2+4)| 3^{-}\right\rangle^{3}[24]}{3\left\langle 5^{-}|(2+3)| 4^{-}\right\rangle\langle 16\rangle\langle 23\rangle\langle 56\rangle[34]^{2} s_{234}} \\
& +\frac{\left\langle 1^{-}|(2+4)| 3^{-}\right\rangle\langle 12\rangle[23]\left(\left\langle 1^{-}|(2+4)| 3^{-}\right\rangle+\langle 12\rangle[23]\right)}{6\langle 16\rangle\langle 23\rangle\langle 25\rangle\langle 56\rangle[34]^{2} s_{234}}
\end{aligned}
$$




$$
\begin{aligned}
& +\frac{\left\langle 1^{-}|(2+4)| 3^{-}\right\rangle^{2}\langle 12\rangle\langle 15\rangle[23]}{2\left\langle 1^{-}|(2+3)| 4^{-}\right\rangle\langle 16\rangle\langle 25\rangle^{2}\langle 56\rangle[34] s_{234}}-\frac{\langle 14\rangle^{4}}{3\langle 12\rangle\langle 23\rangle\langle 34\rangle\langle 45\rangle\langle 56\rangle\langle 61\rangle} \\
& -\frac{\left\langle 5^{-}|(1+4)| 6^{-}\right\rangle\langle 14\rangle\langle 15\rangle[56] s_{146}}{6 s_{16}\left\langle 3^{-}|(1+4)| 6^{-}\right\rangle\left\langle 5^{-}|(2+3)| 4^{-}\right\rangle\langle 23\rangle\langle 25\rangle\langle 56\rangle} \\
& -\frac{\langle 12\rangle\langle 14\rangle^{2}[23]}{6\langle 16\rangle\langle 23\rangle\langle 25\rangle\langle 34\rangle\langle 56\rangle[34]}\left(1+\frac{3\langle 15\rangle\langle 23\rangle}{\langle 13\rangle\langle 25\rangle}\right) \\
& +\frac{\left\langle 2^{-}|(1+4)| 6^{-}\right\rangle^{3}\left\langle 3^{-}|(2+5)| 4^{-}\right\rangle[23][46]}{3\left\langle 2^{-}|(3+5)| 4^{-}\right\rangle^{2}\left\langle 5^{-}|(1+4)| 6^{-}\right\rangle\left\langle 5^{-}|(2+3)| 4^{-}\right\rangle\langle 23\rangle^{2}[14][16]} \\
& +\frac{\langle 14\rangle^{2}\langle 15\rangle^{2}[35]}{6\langle 12\rangle\langle 16\rangle\langle 25\rangle\langle 34\rangle\langle 35\rangle\langle 56\rangle[34]}\left(1+\frac{3\langle 12\rangle\langle 35\rangle}{\langle 13\rangle\langle 25\rangle}\right) \\
& +\frac{\langle 14\rangle\langle 15\rangle[23][56]}{6\left\langle 1^{-}|(3+4)| 2^{-}\right\rangle\langle 25\rangle\langle 56\rangle s_{234}}\left(\frac{\langle 14\rangle}{\langle 23\rangle}+\frac{3\left\langle 1^{-}|(2+4)| 3^{-}\right\rangle\langle 15\rangle}{\left\langle 1^{-}|(2+3)| 4^{-}\right\rangle\langle 25\rangle}\right) \\
& -\frac{\left\langle 1^{-}|(3+5)| 4^{-}\right\rangle^{4}[35]^{3}}{6\left\langle 2^{-}|(3+5)| 4^{-}\right\rangle\left\langle 6^{-}|(3+5)| 4^{-}\right\rangle\langle 12\rangle\langle 16\rangle\langle 35\rangle[34]^{2}[45]^{2} s_{345}} \\
& +\frac{\left\langle 1^{-}|(3+5)| 4^{-}\right\rangle^{4}\langle 26\rangle[26][35]^{4}}{6\left\langle 1^{-}|(3+4)| 5^{-}\right\rangle\left\langle 1^{-}|(4+5)| 3^{-}\right\rangle\left\langle 2^{-}|(3+5)| 4^{-}\right\rangle^{2}\left\langle 6^{-}|(3+5)| 4^{-}\right\rangle^{2}[34][45] s_{345}} \\
& +\frac{\left\langle 1^{-}|(2+6)(3+5)| 6^{+}\right\rangle^{2}\langle 16\rangle[26][35]}{3\left\langle 1^{-}|(3+4)| 5^{-}\right\rangle\left\langle 1^{-}|(4+5)| 3^{-}\right\rangle\left\langle 6^{-}|(3+5)| 4^{-}\right\rangle\langle 26\rangle\langle 36\rangle\langle 56\rangle s_{345}} \\
& \times\left(\frac{\langle 14\rangle}{2\langle 35\rangle}-\frac{\langle 16\rangle[35]}{\left\langle 6^{-}|(3+5)| 4^{-}\right\rangle}\right) \\
& \left\langle 5^{-}|(2+3)| 6^{-}\right\rangle[56] s_{146}^{2}([14]\langle 12\rangle\langle 15\rangle+\langle 25\rangle\langle 16\rangle[46]) \\
& -\frac{}{3\left\langle 2^{-}|(3+5)| 4^{-}\right\rangle\left\langle 3^{-}|(1+4)| 6^{-}\right\rangle\left\langle 5^{-}|(2+3)| 4^{-}\right\rangle\langle 16\rangle\langle 23\rangle\langle 56\rangle[16]\langle 25\rangle[14]} \\
& -\frac{\langle 15\rangle^{2}[23]^{2}[56]}{6\left\langle 1^{-}|(3+4)| 2^{-}\right\rangle\left\langle 5^{-}|(2+3)| 4^{-}\right\rangle\langle 25\rangle\langle 56\rangle s_{234}} \\
& \times\left(3\langle 14\rangle+\frac{3\left\langle 1^{-}|(2+4)| 3^{-}\right\rangle\langle 15\rangle\langle 23\rangle}{\left\langle 1^{-}|(2+3)| 4^{-}\right\rangle\langle 25\rangle}-\frac{2\langle 15\rangle\langle 26\rangle[23]}{\langle 56\rangle[34]}\right) \\
& +\frac{\langle 14\rangle^{3}\langle 15\rangle[25]}{6\left\langle 1^{-}|(3+4)| 2^{-}\right\rangle\left\langle 3^{-}|(1+4)| 6^{-}\right\rangle\langle 13\rangle\langle 16\rangle^{2}\langle 23\rangle\langle 25\rangle^{2}\langle 34\rangle\langle 56\rangle} \\
& \times\left(\left(\left\langle 1^{-}|6(2+5)| 3^{+}\right\rangle+\left\langle 1^{-}|(5+6) 2| 3^{+}\right\rangle\right)(\langle 13\rangle\langle 25\rangle-3\langle 15\rangle\langle 23\rangle)\right. \\
& -3\langle 12\rangle\langle 15\rangle\langle 23\rangle\langle 35\rangle[25]) \\
& +\frac{\langle 14\rangle^{3}\langle 36\rangle[26]}{6\left\langle 1^{-}|(3+4)| 5^{-}\right\rangle\left\langle 3^{-}|(1+4)| 2^{-}\right\rangle\langle 12\rangle\langle 13\rangle\langle 26\rangle^{2}\langle 34\rangle\langle 35\rangle^{2}\langle 56\rangle} \\
& \times\left(2\left(\left\langle 1^{-}|2(5+6)| 3^{+}\right\rangle+\left\langle 1^{-}|(2+6) 5| 3^{+}\right\rangle\right)\langle 13\rangle\langle 26\rangle\right. \\
& \left.-3\left(2\left\langle 1^{-}|2(5+6)| 3^{+}\right\rangle+\left\langle 1^{-}|65| 3^{+}\right\rangle\right)\langle 16\rangle\langle 23\rangle\right) \\
& -\frac{\left\langle 1^{-}|(4+5)| 3^{-}\right\rangle\langle 15\rangle^{2}[35]}{2\left\langle 1^{-}|(3+5)| 4^{-}\right\rangle\langle 12\rangle\langle 16\rangle\langle 23\rangle\langle 25\rangle\langle 35\rangle\langle 56\rangle[34] s_{345}}
\end{aligned}
$$




$$
\begin{aligned}
& \times\left(\frac{\left\langle 1^{-}|(2+6)(3+5)| 2^{+}\right\rangle\langle 12\rangle\langle 35\rangle}{\langle 25\rangle}-\left\langle 1^{-}|(3+4)| 5^{-}\right\rangle\langle 13\rangle\langle 25\rangle\right) \\
& +\frac{\left\langle 2^{-}|(1+4)| 6^{-}\right\rangle s_{146}^{2}}{3 s_{16}\left\langle 2^{-}|(3+5)| 4^{-}\right\rangle^{2}\left\langle 3^{-}|(1+4)| 6^{-}\right\rangle\langle 23\rangle\langle 25\rangle\langle 26\rangle[14]} \\
& \times\left(\frac{\langle 16\rangle\langle 25\rangle\langle 26\rangle[46]^{2}[56]}{\left\langle 5^{-}|(2+3)| 4^{-}\right\rangle}-\frac{\left\langle 2^{-}|(1+4)| 6^{-}\right\rangle\langle 12\rangle^{2}[14][26]}{\left\langle 5^{-}|(2+3)| 6^{-}\right\rangle}\right) \\
& +\frac{\left\langle 2^{-}|(1+4)| 6^{-}\right\rangle^{2}\langle 12\rangle[26] s_{146}}{2 s_{16}\left\langle 2^{-}|(3+5)| 4^{-}\right\rangle\left\langle 3^{-}|(1+4)| 6^{-}\right\rangle\left\langle 5^{-}|(1+4)| 6^{-}\right\rangle\langle 23\rangle\langle 25\rangle\langle 26\rangle\langle 56\rangle[46]} \\
& \times\left(\frac{\left\langle 5^{-}|(1+4)| 6^{-}\right\rangle\langle 15\rangle\langle 26\rangle}{\langle 25\rangle}-\frac{\left\langle 6^{-}|(1+4)| 6^{-}\right\rangle\langle 16\rangle\langle 25\rangle}{\langle 26\rangle}+\frac{\langle 14\rangle\langle 56\rangle[46]}{3}\right) \\
& +\frac{\left\langle 1^{-}|(2+6)(3+5)| 6^{+}\right\rangle^{2}\langle 16\rangle[26][35]}{\left\langle 1^{-}|(3+4)| 5^{-}\right\rangle\left\langle 1^{-}|(3+5)| 4^{-}\right\rangle\left\langle 1^{-}|(4+5)| 3^{-}\right\rangle\left\langle 6^{-}|(3+5)| 4^{-}\right\rangle\langle 23\rangle\langle 26\rangle\langle 35\rangle\langle 36\rangle\langle 56\rangle} \\
& \times \frac{1}{2 s_{345}}\left(\frac{\left\langle 1^{-}|(3+4)| 5^{-}\right\rangle\langle 13\rangle\langle 26\rangle\langle 35\rangle}{\langle 36\rangle}-\frac{\left\langle 1^{-}|(2+6)(3+5)| 2^{+}\right\rangle\langle 12\rangle\langle 36\rangle}{\langle 26\rangle}\right) \\
& +\frac{\langle 14\rangle^{3}\langle 12\rangle\langle 23\rangle}{\langle 34\rangle\langle 56\rangle\langle 61\rangle^{2}\langle 13\rangle\langle 35\rangle^{2}\left\langle 1^{-}|(3+4)| 5^{-}\right\rangle\left\langle 3^{-}|(1+4)| 6^{-}\right\rangle} \\
& \times\left(\frac{1}{3} \frac{\langle 13\rangle^{2}\langle 35\rangle^{2}\langle 16\rangle^{2}[25]^{2}[26]^{2}}{\langle 12\rangle\langle 23\rangle\left\langle 1^{-}|(3+4)| 2^{-}\right\rangle\left\langle 3^{-}|(1+4)| 2^{-}\right\rangle}-\frac{1}{3} \frac{\langle 13\rangle\langle 15\rangle^{2}\langle 35\rangle^{2}[25]^{3}}{\langle 23\rangle\langle 25\rangle\left\langle 1^{-}|(3+4)| 2^{-}\right\rangle}\right. \\
& -\frac{1}{3} \frac{\langle 13\rangle\langle 36\rangle^{2}\langle 16\rangle^{2}[26]^{3}}{\langle 12\rangle\langle 26\rangle\left\langle 3^{-}|(1+4)| 2^{-}\right\rangle} \\
& +\frac{1}{3} \frac{\langle 13\rangle}{\langle 12\rangle\langle 23\rangle\langle 25\rangle\langle 26\rangle}\left(\left\langle 1^{-}|(2+6) 5| 3^{+}\right\rangle+\left\langle 1^{-}|6(2+5)| 3^{+}\right\rangle\right) \\
& \times(\langle 16\rangle\langle 36\rangle\langle 25\rangle[26]+\langle 15\rangle\langle 35\rangle\langle 26\rangle[25]) \\
& +\frac{1}{2}[25][26]\langle 61\rangle\langle 35\rangle\left(\frac{1}{3} \frac{\langle 13\rangle^{2}}{\langle 12\rangle\langle 23\rangle}+\frac{\langle 15\rangle\langle 35\rangle}{\langle 25\rangle^{2}}+\frac{\langle 13\rangle\langle 35\rangle}{\langle 23\rangle\langle 25\rangle}+\frac{\langle 16\rangle\langle 36\rangle}{\langle 26\rangle^{2}}\right. \\
& \left.+\frac{\langle 13\rangle\langle 16\rangle}{\langle 12\rangle\langle 26\rangle}\right) \\
& +\frac{\left\langle 1^{-}|(2+6) 5| 3^{+}\right\rangle\langle 35\rangle^{2}[25]}{\langle 25\rangle^{2}} \frac{(\langle 15\rangle\langle 23\rangle+\langle 13\rangle\langle 25\rangle)}{\langle 23\rangle^{2}} \\
& +\frac{\left\langle 1^{-}|6(2+5)| 3^{+}\right\rangle\langle 16\rangle^{2}[26]}{\langle 26\rangle^{2}} \frac{(\langle 12\rangle\langle 36\rangle+\langle 13\rangle\langle 26\rangle)}{\langle 12\rangle^{2}} \\
& +\frac{\langle 13\rangle^{2}}{\langle 12\rangle^{2}\langle 23\rangle^{2}}\left(\left\langle 1^{-}|(2+6) 5| 3^{+}\right\rangle\left\langle 1^{-}|25| 3^{+}\right\rangle+\left\langle 1^{-}|62| 3^{+}\right\rangle\left\langle 1^{-}|25| 3^{+}\right\rangle\right. \\
& \left.\left.\left.+\left\langle 1^{-}|6(2+5)| 3^{+}\right\rangle\left\langle 1^{-}|62| 3^{+}\right\rangle\right)\right)\right] .
\end{aligned}
$$


[1] A. Denner, S. Dittmaier, M. Roth and L. H. Wieders, Phys. Lett. B612:223 (2005) [hepph/0502063]; Nucl. Phys. B724:247 (2005) [hep-ph/0505042].

[2] W. T. Giele and E. W. N. Glover, JHEP 0404:029 (2004) [hep-ph/0402152];

R. K. Ellis, W. T. Giele and G. Zanderighi, Phys. Rev. D72:054018 (2005) [hep-ph/0506196].

[3] R. K. Ellis, W. T. Giele and G. Zanderighi, Phys. Rev. D73:014027 (2006) [hep-ph/0508308].

[4] R. K. Ellis, W. T. Giele and G. Zanderighi, JHEP 0605:027 (2006) [hep-ph/0602185].

[5] T. Binoth, G. Heinrich and N. Kauer, Nucl. Phys. B654:277 (2003) [hep-ph/0210023];

M. Kramer and D. E. Soper, Phys. Rev. D66:054017 (2002) [hep-ph/0204113];

Z. Nagy and D. E. Soper, JHEP 0309:055 (2003) [hep-ph/0308127];

T. Binoth, J. P. Guillet, G. Heinrich, E. Pilon and C. Schubert, JHEP 0510:015 (2005) [hepph/0504267];

C. Anastasiou and A. Daleo, hep-ph/0511176;

M. Czakon, hep-ph/0511200;

T. Binoth, M. Ciccolini and G. Heinrich, Nucl. Phys. Proc. Suppl. 157:48 (2006) [hep$\mathrm{ph} / 0601254]$.

[6] Z. Bern, L. J. Dixon, D. C. Dunbar and D. A. Kosower, Nucl. Phys. B425:217 (1994) [hepph/9403226].

[7] Z. Bern, L. J. Dixon, D. C. Dunbar and D. A. Kosower, Nucl. Phys. B435:59 (1995) [hepph/9409265].

[8] Z. Bern and A. G. Morgan, Nucl. Phys. B467:479 (1996) [hep-ph/9511336];

Z. Bern, L. J. Dixon, D. C. Dunbar and D. A. Kosower, Phys. Lett. B394:105 (1997) [hepth/9611127].

[9] Z. Bern, L. J. Dixon and D. A. Kosower, Ann. Rev. Nucl. Part. Sci. 46:109 (1996) [hep$\mathrm{ph} / 9602280]$.

[10] Z. Bern, L. J. Dixon and D. A. Kosower, Nucl. Phys. Proc. Suppl. 51C:243 (1996) [hepph/9606378];

Z. Bern, L. J. Dixon and D. A. Kosower, JHEP 0001:027 (2000) [hep-ph/0001001];

Z. Bern, A. De Freitas and L. J. Dixon, JHEP 0203:018 (2002) [hep-ph/0201161].

[11] L. D. Landau, Nucl. Phys. 13:181 (1959); 
S. Mandelstam, Phys. Rev. 112:1344 (1958);

R. E. Cutkosky, J. Math. Phys. 1:429 (1960);

R. J. Eden, P. V. Landshoff, D. I. Olive, J. C. Polkinghorne, The Analytic S Matrix, (Cambridge University Press, 1966).

[12] G. 't Hooft and M. Veltman, Nucl. Phys. B44:189 (1972).

[13] A. Brandhuber, S. McNamara, B. Spence and G. Travaglini, JHEP 0510:011 (2005) [hepth/0506068].

[14] W. L. van Neerven, Nucl. Phys. B268:453 (1986).

[15] F. Cachazo, P. Svrček and E. Witten, JHEP 0409:006 (2004) [hep-th/0403047];

C.-J. Zhu, JHEP 0404:032 (2004) [hep-th/0403115];

G. Georgiou and V. V. Khoze, JHEP 0405:070 (2004) [hep-th/0404072];

J.-B. Wu and C.-J. Zhu, JHEP 0407:032 (2004) [hep-th/0406085]; JHEP 0409:063 (2004) [hepth/0406146];

D. A. Kosower, Phys. Rev. D71:045007 (2005) [hep-th/0406175];

G. Georgiou, E. W. N. Glover and V. V. Khoze, JHEP 0407:048 (2004) [hep-th/0407027];

Y. Abe, V. P. Nair and M. I. Park, Phys. Rev. D71:025002 (2005) [hep-th/0408191];

L. J. Dixon, E. W. N. Glover and V. V. Khoze, JHEP 0412:015 (2004) [hep-th/0411092];

Z. Bern, D. Forde, D. A. Kosower and P. Mastrolia, Phys. Rev. D72:025006 (2005) [hep$\mathrm{ph} / 0412167]$;

T. G. Birthwright, E. W. N. Glover, V. V. Khoze and P. Marquard, JHEP 0505:013 (2005) [hep-ph/0503063]; JHEP 0507:068 (2005) [hep-ph/0505219].

[16] K. Risager, JHEP 0512:003 (2005) [hep-th/0508206].

[17] A. Brandhuber, B. Spence and G. Travaglini, Nucl. Phys. B706:150 (2005) [hep-th/0407214]; C. Quigley and M. Rozali, JHEP 0501:053 (2005) [hep-th/0410278];

J. Bedford, A. Brandhuber, B. Spence and G. Travaglini, Nucl. Phys. B706:100 (2005) [hepth/0410280].

[18] J. Bedford, A. Brandhuber, B. Spence and G. Travaglini, Nucl. Phys. B712:59 (2005) [hepth/0412108].

[19] F. Cachazo, hep-th/0410077;

R. Britto, F. Cachazo and B. Feng, Phys. Rev. D71:025012 (2005) [hep-th/0410179].

[20] R. Britto, F. Cachazo and B. Feng, Nucl. Phys. B725:275 (2005) [hep-th/0412103]. 
[21] Z. Bern, L. J. Dixon and D. A. Kosower, Nucl. Phys. B513:3 (1998) [hep-ph/9708239].

[22] Z. Bern, L. J. Dixon and D. A. Kosower, JHEP 0408:012 (2004) [hep-ph/0404293].

[23] Z. Bern, V. Del Duca, L. J. Dixon and D. A. Kosower, Phys. Rev. D71:045006 (2005) [hepth/0410224].

[24] R. Britto, E. Buchbinder, F. Cachazo and B. Feng, Phys. Rev. D72:065012 (2005) [hep$\mathrm{ph} / 0503132]$.

[25] R. Britto, B. Feng and P. Mastrolia, Phys. Rev. D73:105004 (2006) [hep-ph/0602178].

[26] Z. Bern, L. J. Dixon and D. A. Kosower, Phys. Rev. Lett. 70:2677 (1993) [hep-ph/9302280].

[27] S. J. Bidder, N. E. J. Bjerrum-Bohr, L. J. Dixon and D. C. Dunbar, Phys. Lett. B606:189 (2005) [hep-th/0410296].

[28] S. J. Bidder, N. E. J. Bjerrum-Bohr, D. C. Dunbar and W. B. Perkins, Phys. Lett. B608:151 (2005) [hep-th/0412023].

[29] Z. Bern, N. E. J. Bjerrum-Bohr, D. C. Dunbar and H. Ita, JHEP 0511:027 (2005) [hep$\mathrm{ph} / 0507019]$.

[30] L. J. Dixon and A. Signer, Phys. Rev. D56:4031 (1997) [hep-ph/9706285];

Z. Nagy and Z. Trócsányi, Phys. Rev. D59:014020 (1999) [Erratum-ibid. D62:099902 (2000)] [hep-ph/9806317];

R. K. Ellis and S. Veseli, Phys. Rev. D60:011501 (1999) [hep-ph/9810489];

S. Weinzierl and D. A. Kosower, Phys. Rev. D60:054028 (1999) [hep-ph/9901277];

J. M. Campbell and R. K. Ellis, Phys. Rev. D62:114012 (2000) [hep-ph/0006304];

M. Cacciari, V. Del Duca, S. Frixione and Z. Trócsányi, JHEP 0102:029 (2001) [hep$\mathrm{ph} / 0011368]$

Z. Nagy and Z. Trócsányi, Phys. Rev. Lett. 87:082001 (2001) [hep-ph/0104315];

J. Campbell and R. K. Ellis, Phys. Rev. D65:113007 (2002) [hep-ph/0202176].

[31] Z. Bern, L. J. Dixon and D. A. Kosower, Phys. Rev. D73:065013 (2006) [hep-ph/0507005].

[32] C. F. Berger, Z. Bern, L. J. Dixon, D. Forde and D. A. Kosower, hep-ph/0604195.

[33] R. Britto, F. Cachazo and B. Feng, Nucl. Phys. B715:499 (2005) [hep-th/0412308].

[34] Z. Bern, L. J. Dixon and D. A. Kosower, Phys. Rev. D72:045014 (2005) [hep-th/0412210].

[35] R. Roiban, M. Spradlin and A. Volovich, Phys. Rev. Lett. 94:102002 (2005) [hep-th/0412265].

[36] W. T. Giele and E. W. N. Glover, Phys. Rev. D46:1980 (1992);

Z. Kunszt, A. Signer and Z. Trócsányi, Nucl. Phys. B420:550 (1994) [hep-ph/9401294]; 
S. Catani, Phys. Lett. B427:161 (1998) [hep-ph/9802439].

[37] E. Witten, Commun. Math. Phys. 252:189 (2004) [hep-th/0312171].

[38] R. Britto, F. Cachazo, B. Feng and E. Witten, Phys. Rev. Lett. 94:181602 (2005) [hepth/0501052].

[39] M.-X. Luo and C.-K. Wen, JHEP 0503:004 (2005) [hep-th/0501121]; Phys. Rev. D71:091501 (2005) [hep-th/0502009].

[40] R. Britto, B. Feng, R. Roiban, M. Spradlin and A. Volovich, Phys. Rev. D71:105017 (2005) [hep-th/0503198]

[41] K. J. Ozeren and W. J. Stirling, JHEP 0511:016 (2005) [hep-th/0509063];

M. Dinsdale, M. Ternick and S. Weinzierl, JHEP 0603:056 (2006) [hep-ph/0602204];

D. de Florian and J. Zurita, JHEP 0605:073 (2006) [hep-ph/0605291].

[42] S. D. Badger, E. W. N. Glover, V. V. Khoze and P. Svrček, JHEP 0507:025 (2005) [hepth/0504159].

[43] S. D. Badger, E. W. N. Glover and V. V. Khoze, JHEP 0601:066 (2006) [hep-th/0507161];

C. Schwinn and S. Weinzierl, JHEP 0603:030 (2006) [hep-th/0602012];

P. Ferrario, G. Rodrigo and P. Talavera, Phys. Rev. Lett. 96:182001 (2006) [hep-th/0602043].

[44] D. Forde and D. A. Kosower, Phys. Rev. D73:065007 (2006) [hep-th/0507292].

[45] Z. Bern, L. J. Dixon and D. A. Kosower, Phys. Rev. D71:105013 (2005) [hep-th/0501240].

[46] Z. Bern, L. J. Dixon and D. A. Kosower, Phys. Rev. D72:125003 (2005) [hep-ph/0505055].

[47] D. Forde and D. A. Kosower, Phys. Rev. D73:061701 (2006) [hep-ph/0509358].

[48] M. L. Mangano and S. J. Parke, Phys. Rept. 200:301 (1991);

L. J. Dixon, in QCD \& Beyond: Proceedings of TASI '95, ed. D. E. Soper (World Scientific, 1996) [hep-ph/9601359].

[49] Z. Bern and G. Chalmers, Nucl. Phys. B447:465 (1995) [hep-ph/9503236].

[50] D. A. Kosower, Nucl. Phys. B552:319 (1999) [hep-ph/9901201];

D. A. Kosower and P. Uwer, Nucl. Phys. B563:477 (1999) [hep-ph/9903515].

[51] Z.-G. Xiao, G. Yang and C.-J. Zhu, hep-ph/0607015;

X. Su, Z.-G. Xiao, G. Yang and C.-J. Zhu, hep-ph/0607016.

[52] Z.-G. Xiao, G. Yang and C.-J. Zhu, hep-ph/0607017.

[53] F. A. Berends, R. Kleiss, P. De Causmaecker, R. Gastmans and T. T. Wu, Phys. Lett. B103:124 (1981); 
P. De Causmaecker, R. Gastmans, W. Troost and T. T. Wu, Nucl. Phys. B206:53 (1982);

Z. Xu, D. H. Zhang and L. Chang, TUTP-84/3-TSINGHUA;

R. Kleiss and W. J. Stirling, Nucl. Phys. B262:235 (1985);

J. F. Gunion and Z. Kunszt, Phys. Lett. B161:333 (1985);

Z. Xu, D. H. Zhang and L. Chang, Nucl. Phys. B291:392 (1987).

[54] J. E. Paton and H. M. Chan, Nucl. Phys. B10:516 (1969);

P. Cvitanović, P. G. Lauwers and P. N. Scharbach, Nucl. Phys. B186:165 (1981);

D. Kosower, B. H. Lee and V. P. Nair, Phys. Lett. B201:85 (1988).

[55] Z. Bern and D. A. Kosower, Nucl. Phys. B362:389 (1991).

[56] Z. Bern and D. A. Kosower, Nucl. Phys. B379:451 (1992).

[57] S. Catani, M. H. Seymour and Z. Trócsányi, Phys. Rev. D55:6819 (1997) [hep-ph/9610553];

Z. Bern, A. De Freitas, L. J. Dixon and H. L. Wong, Phys. Rev. D66:085002 (2002) [hep$\mathrm{ph} / 0202271]$.

[58] Z. Bern, L. J. Dixon and D. A. Kosower, hep-th/9311026;

Z. Bern, G. Chalmers, L. J. Dixon and D. A. Kosower, Phys. Rev. Lett. 72:2134 (1994) [hep-ph/9312333].

[59] G. Mahlon, Phys. Rev. D49:4438 (1994) [hep-ph/9312276].

[60] R. Kleiss and H. Kuijf, Nucl. Phys. B312:616 (1989).

[61] V. Del Duca, L. J. Dixon and F. Maltoni, Nucl. Phys. B571:51 (2000) [hep-ph/9910563].

[62] S. J. Parke and T. R. Taylor, Phys. Rev. Lett. 56:2459 (1986).

[63] D. Vaman and Y. P. Yao, JHEP 0604:030 (2006) [hep-th/0512031];

P. D. Draggiotis, R. H. P. Kleiss, A. Lazopoulos and C. G. Papadopoulos, hep-ph/0511288.

[64] W. T. Giele and E. W. N. Glover, Phys. Rev. D46:1980 (1992);

W. T. Giele, E. W. N. Glover and D. A. Kosower, Nucl. Phys. B403:633 (1993) [hep$\mathrm{ph} / 9302225]$;

S. Frixione, Z. Kunszt and A. Signer, Nucl. Phys. B467:399 (1996) [hep-ph/9512328];

S. Catani and M. H. Seymour, Nucl. Phys. 485:291 (1997) [Erratum-ibid. B510:503 (1997)] [hep-ph/9605323];

D. A. Kosower, Phys. Rev. D 57:5410 (1998) [hep-ph/9710213].

[65] F. Cachazo, P. Svrček and E. Witten, JHEP 0410:074 (2004) [hep-th/0406177].

[66] I. Bena, Z. Bern, D. A. Kosower and R. Roiban, Phys. Rev. D71:106010 (2005) [hep- 
th/0410054].

[67] F. Cachazo, P. Svrček and E. Witten, JHEP 0410:077 (2004) [hep-th/0409245].

[68] R. Britto, F. Cachazo and B. Feng, Phys. Lett. B611:167 (2005) [hep-th/0411107].

[69] F. A. Berends and W. Giele, Nucl. Phys. B294:700 (1987).

[70] M. L. Mangano, S. J. Parke and Z. Xu, Nucl. Phys. B298:653 (1988).

[71] Z. Kunszt and W. J. Stirling, Phys. Rev. D37:2439 (1988).

[72] C. J. Maxwell, Phys. Lett. B192:190 (1987); Nucl. Phys. B316:321 (1989);

M. L. Mangano and S. J. Parke, Phys. Rev. D39:758 (1989).

[73] D. Binosi and L. Theussl, Comput. Phys. Commun. 161:76 (2004) [hep-ph/0309015].

[74] J. A. M. Vermaseren, Comput. Phys. Commun. 83:45 (1994). 